\title{
Produto fibrado orientado de álgebras e dimensão de representação
}

\author{
Heily Wagner
}

TESE APRESENTADA

$\mathrm{AO}$

Instituto DE MATEMÁtica E EstatísticA

DA

Universidade DE SÃo PAUlo

PARA

OBTENÇÃO DO TÍTULO

$\mathrm{DE}$

DOUTOR EM CIÊNCIAS

\author{
Programa: Matemática \\ Orientador: Prof. Dr. Flávio Ulhoa Coelho
}

Durante o desenvolvimento deste trabalho a autora recebeu auxílio financeiro do CNPq

São Paulo, outubro de 2012 


\title{
Produto fibrado orientado de álgebras e dimensão de representação
}

\author{
Esta tese contém as correções e alterações \\ sugeridas pela Comissão Julgadora durante a defesa \\ realizada por Heily Wagner em 31/08/2012. \\ O original encontra-se disponível no Instituto de \\ Matemática e Estatística da Universidade de São Paulo.
}

Comissão Julgadora:

- Prof. Dr. Flávio Ulhoa Coelho (orientador) - IME-USP

- Prof. Dr. Eduardo do Nascimento Marcos - IME-USP

- Prof. Dra. Diane Castonguay - UFG

- Prof. Dr. Viktor Bekkert - UFMG

- Prof. Dr. Edson Ribeiro Alvares - UFPR 


\section{Agradecimentos}

Gostaria de agradecer, primeiramente, ao meu orientador, professor Flávio Ulhoa Coelho, por seus ensinamentos, por sua paciência e, principalmente, pelo apoio e pela confiança, ao longo dos últimos anos.

Gostaria de agradecer ao professor Ibrahim Assem pelos seus ensinamentos enquanto eu estive em Sherbrooke, Canadá. Boa parte dos resultados dessa tese foram desenvolvidos durante este estágio Sanduíche de um ano. Agradeço também, à Université de Sherbrooke, que me acolheu por esse período, dando todo o apoio necessário, como se eu fosse uma aluna de seu instituto.

Agradeço ao Francisco Medeiros, pelo apoio, cumplicidade e companheirismo. E, também, pela verificação de algumas contas e pela elaboração de algumas aljavas.

Finalmente, gostaria de agradecer minha família, em especial minha mãe, Ivone Martins Nogueira Wagner, que sempre apoiou meus estudos. 


\section{Resumo}

\section{WAGNER, H. Produto fibrado orientado de álgebras e dimensão de representação.}

Tese (Doutorado) - Instituto de Matemática e Estatística, Universidade de São Paulo, São Paulo, 2012.

Dados dois epimorfismos de álgebras $f: A \rightarrow B$ e $g: C \rightarrow B$, o produto fibrado é a subálgebra $R$ de $A \times C$ definida por $\{(a, c) \in A \times C \mid f(a)=g(c)\}$. Para álgebras básicas de dimensão finita sobre um corpo algebricamente fechado $k$, que podem ser determinadas por aljavas com relações, a aljava ordinária do produto fibrado $R$ pode ser determinada pelas respectivas aljavas de $A$ e $C$. Estudamos essencialmente um tipo especial de produto fibrado, no qual a aljava ordinária tem uma certa orientação, o chamado produto fibrado orientado. Definimos ainda, o produto fibrado Dynkin orientado no qual a aljava da álgebra $B$ é de tipo Dynkin com um único poço. Para esses casos, estudamos características do produto fibrado $R$ a partir de informações de $A$ e de $C$, tais como as aljavas de Auslander-Reiten, classes de álgebras (hereditária, shod, inclinada, etc) e, em especial, a dimensão de representação. Para a dimensão de representação, além do estudo para produtos fibrados orientados, mostramos que se pudermos dividir a categoria de módulos indecomponíveis de uma álgebra de Artin em pedaços com certas propriedades, então é possível estimar a dimensão de representação da álgebra através do cálculo dessa invariante de álgebras associadas a cada um desses pedaços. Essa técnica nos permitiu calcular a dimensão de representação das álgebras ada, por exemplo.

Palavras-chave: produto fibrado, dimensão de representação, propriedades homológicas. 


\section{Abstract}

WAGNER, H. Oriented pullback of algebras and representation dimension. Tese (Doutorado) - Instituto de Matemática e Estatística, Universidade de São Paulo, São Paulo, 2012 .

Given two epimorphisms of algebras $f: A \rightarrow B$ and $g: C \rightarrow B$, the pullback $R$ is the subalgebra of $A \times C$ defined by $\{(a, c) \in A \times C \mid f(a)=g(c)\}$. For finite dimensional basic $k$-algebras (where $k$ is an algebraically closed field), which can be determined by bounded quivers, the ordinary quiver of the pullback $R$ can be determined by those of $A, B$ and $C$. We study a more specific case, the oriented pullback, where the quiver has a certain orientation. We also define the Dynkin oriented pullback, whose main feature is the ordinary quiver of $B$ being a Dynkin with a single sink. For these cases, we studied the characteristics of the pullback $R$ from information of $A$ and $C$, such as the Auslander-Reiten quivers, class of algebras (hereditary, shod, tilted, etc) and especially the representation dimension. For representation dimension, in addition to the study for oriented pullbacks, we show that if we divide the category of indecomposable modules into pieces with certain characteristics, we can calculate the representation dimension of this algebra by calculating this invariant of algebras associated to each piece. This technique allowed us to calculate the representation dimension of the ada algebras, for instance.

Keywords: pullback, representation dimension, homologic properties. 


\section{Sumário}

$\begin{array}{ll}\text { Introdução } & 1\end{array}$

1 Preliminares $\quad 7$

1.1 Aljavas e Representações . . . . . . . . . . . . . . . . . . . . 7

1.2 Teoria de Auslander-Reiten . . . . . . . . . . . . . . . . . . . . 11

1.3 Subcategorias especiais . . . . . . . . . . . . . . . . . . . . 13

1.4 Algumas classes de álgebras . . . . . . . . . . . . . . . . . . . . . . 14

2 Dynkin com um único poço $\quad 17$

2.1 Lista das aljavas de tipo Dynkin com um único poço . . . . . . . . . . . . . 17

2.2 Dynkin com um único poço e módulos injetivos . . . . . . . . . . . . . . . . 19

3 Produto fibrado de álgebras $\quad 31$

3.1 Produto fibrado de álgebras e de módulos . . . . . . . . . . . . . . . . 31

3.1 .1 Módulos sobre o produto fibrado . . . . . . . . . . . . . 36

3.1 .2 Caso estudado: aljavas . . . . . . . . . . . . . . . . . 38

3.1.3 Aljava ordinária do produto fibrado . . . . . . . . . . . . . 39

3.2 Produto fibrado orientado . . . . . . . . . . . . . . . . . . . 41

3.2.1 Módulos sobre um produto fibrado orientado . . . . . . . . . . . . 43

3.2.2 Projetivos, Injetivos e Dimensão global . . . . . . . . . . . . . . . . 44

3.3 Casos particulares do produto fibrado orientado . . . . . . . . . . . . . . 47

3.3.1 Produto fibrado Dynkin orientado . . . . . . . . . . . . . 48

4 Produto fibrado orientado e subcategorias de ind $R \quad 53$

4.1 Produto fibrado das álgebras suporte . . . . . . . . . . . . . . . . . . 53

4.2 Subcategorias especiais no produto fibrado . . . . . . . . . . . . . 57

4.3 Aljava de Auslander-Reiten do produto fibrado orientado . . . . . . . . . 59

4.4 Produto fibrado orientado e classes de algebras . . . . . . . . . . . . . 63

5 Dimensão de representação $\quad 67$

5.1 Resolução de aproximação . . . . . . . . . . . . . . . . . . . . . . 67

5.2 Dimensão de representação . . . . . . . . . . . . . . . . . . . . . . 70

5.3 Dimensão de representação e álgebras suporte . . . . . . . . . . . . . . 71

5.4 Dimensão de representação e partes direita e esquerda . . . . . . . . . . . . . 74 
5.5 Dimensão de representação das álgebras laura e ada . . . . . . . . . . . . . . . 84

5.6 Aplicações ao produto fibrado orientado . . . . . . . . . . . . . . . 85

$\begin{array}{lr}\text { A Exemplo } & 89\end{array}$

$\begin{array}{ll}\text { Referências Bibliográficas } & 91\end{array}$ 


\section{Introdução}

As pesquisas em representações de álgebras de Artin centralizam-se no estudo e classificação das álgebras a partir da análise aprofundada das suas respectivas categoria de módulos. Para tanto, diversas metodologias tem sido desenvolvidas nos últimos 30 anos. Mencionamos particularmente as teorias de Auslander-Reiten e de inclinação.

Uma estratégia bem sucedida é a de transferir informações de uma classe de álgebras a outras a partir de certas condições. Um exemplo é o processo inclinante com o qual é possível passar informações da bem estudada classe de álgebras hereditárias para uma classe maior de álgebras chamadas álgebras inclinadas [HR82]. Nesse caso, os focos principais são não só as propriedades homológicas, como dimensão projetiva, injetiva e global, mas também a chamada Teoria de Auslander-Reiten. Tendo isso como base, surgem de forma natural outras classes de álgebras como quase inclinadas [HRS96], shod [CL99], fracamente shod [CL03], laura [AC03], etc.

Neste trabalho, o interesse foi um outro tipo de construção, o chamado produto fibrado. Dados dois epimorfismos $f: A \rightarrow B$ e $g: C \rightarrow B \operatorname{com} A, B$ e $C$ álgebras podemos construir uma nova álgebra $R$ dada por $\{(a, c) \in A \times C \mid f(a)=g(c)\}$. Esta álgebra é chamada de produto fibrado do par $(f, g)$.

O produto fibrado de anéis e de álgebras tem sido estudado a partir de vários pontos de vista [FV85, IPTZ87, Lev08, Mil71, Wis85] mas poucos trabalhos envolvem técnicas recentes da teoria de representações de álgebras.

A ideia inicial é o estudo do produto fibrado de álgebras dadas por quivers com relações como aparece por exemplo em [IPTZ87] e [Lev08]. Nestes trabalhos há, por exemplo, uma descrição, sob certas condições, das aljavas ordinária e de Auslander-Reiten de $R$ a partir das respectivas aljavas de $A, B$ e $C$. Nesses dois trabalhos são estudados produtos fibrados tais que, dentre outras características, a aljava ordinária da álgebra $B$ é de tipo $\mathbb{A}_{n}$ linearmente orientada, que é, em particular, uma álgebra de Nakayama e dá nome ao produto fibrado estudado em [Lev08], o chamado produto fibrado Nakayama orientado.

Uma pergunta natural é se é possível determinar a qual classe de álgebras o produto fibrado $R$ pertence conhecendo-se as classes das álgebras $A, B$ e $C$. Como exemplo, podemos citar um resultado nesta direção devido a Lévesque, [Lev08](2.4.7), que diz que para um produto fibrado Nakayama orientado $R$ de $A \rightarrow B$ e $C \rightarrow B$, se $A$ e $C$ são álgebras hereditárias então $R$ é uma álgebra inclinada. Chegamos a uma generalização desse resultado, para um produto fibrado orientado com certas propriedades (Teorema 4.4.4). 
Nesse trabalho, definimos também um produto fibrado, chamado produto fibrado Dynkin orientado, que engloba os conceitos de Nakayama orientado e o definido em [IPTZ87], dito com condições *. Para esse produto fibrado, mostramos o seguinte resultado que generaliza [IPTZ87](3.5).

Teorema 1. Se $R$ é o produto fibrado Dynkin orientado de $A \rightarrow B$ e $C \rightarrow B$ então ind $R=$ ind $A \cup \operatorname{ind} C$.

Em [IPTZ87](3.5), o produto fibrado estudado tem como principal característica que a álgebra $B$ é de tipo $\mathbb{A}_{n}$ linearmente orientada, enquanto que no caso mais geral de um produto fibrado Dynkin orientado, a álgebra $B$ é de tipo Dynkin com um único poço. Para chegarmos ao Teorema 1 precisamos da seguinte relação entre uma aljava desse tipo e módulos injetivos.

Teorema 2. Seja $Q$ uma aljava de tipo Dynkin com um único poço.

Se $M=\left(\left(V_{i}\right)_{i \in Q_{0}},\left(f_{\alpha}\right)_{\alpha \in Q_{1}}\right)$ é uma $Q$-representação com $f_{\alpha}$ sobrejetora para todo $\alpha$ em $Q_{1}$, então $M$ é a representação de um módulo injetivo.

Para o produto fibrado Nakayama orientado, Lévesque mostrou também que a aljava de Auslander-Reiten de $R$ pode ser determinada a partir das aljavas de Auslander-Reiten de $A$, $B$ e $C$, mais especificamente, $\Gamma_{R}$ é a soma amalgamada das inclusões $\Gamma_{B} \rightarrow \Gamma_{A}$ e $\Gamma_{B} \rightarrow \Gamma_{C}$. Nesse caso, a categoria de módulos indecomponíveis ind $R$ possui uma boa divisão a partir de $\operatorname{ind} A$, ind $B$ e ind $C$. Na verdade, temos para ind $R$ uma triseç̧ão ([AAC $\left.\left.{ }^{+} 11\right]\right)$ da seguinte forma: (ind $A \backslash \operatorname{ind} B$, ind $B$, ind $C \backslash \operatorname{ind} B$ ). Ao contrário do que esperavamos, esses resultados não são verdadeiros para o produto fibrado Dynkin orientado (Ver Exemplo 4.3.2).

Com o intuito de generalizar alguns dos resultados de [IPTZ87], [Lev04] e [Lev08], passamos a estudar o produto fibrado orientado de $A \rightarrow B$ e $C \rightarrow B$. Nesse estudo, encontramos subcategorias especiais $\mathcal{A}$ e $\mathcal{C}$ em ind $R$ definidas, respectivamente, como sendo os predecessores de certos $A$-módulos injetivos e os sucessores de certos $C$-módulos projetivos. Essas subcategorias determinam algumas trisecções de ind $R$. A partir do estudo dessas subcategorias, chegamos a diversos resultados. Por exemplo, o seguinte teorema.

Teorema 3. Seja $R$ o produto fibrado orientado de $A \rightarrow B$ e $C \rightarrow B$ e suponha que $\mathcal{A}$ e $\mathcal{C}$ são disjuntas. Então, existem $\Sigma_{A}$ uma subaljava plena de $\Gamma_{A}, \Sigma_{B}$ uma subaljava plena de $\Gamma_{B}$ e $\Sigma_{C}$ uma subaljava plena de $\Gamma_{C}$ tais que $\Gamma_{R}$ é a soma amalgamada $\Sigma_{A} \coprod_{\Sigma_{B}} \Sigma_{C}$ das inclusões $\Sigma_{B} \rightarrow \Sigma_{A}$ e $\Sigma_{B} \rightarrow \Sigma_{C}$.

Denotamos por $\mathcal{L}_{\Lambda}$ e $\mathcal{R}_{\Lambda}$, respectivamente, a parte esquerda e a parte direita da categoria de módulos como em [HRS96]. Se $\Lambda$ é uma álgebra de dimensão finita de tipo ada ([ACLV12]), ou seja, uma álgebra tal que todos os projetivos e injetivos indecomponíveis estão em $\mathcal{L}_{\Lambda} \cup \mathcal{R}_{\Lambda}$, então $\Lambda$ pode ser vista como um produto fibrado orientado das álgebras suportes, definidas como em [Ass09], determinadas por $\mathcal{R}_{\Lambda}$ e $\mathcal{L}_{\Lambda}$. 
Também estudamos as relações entre as partes direita e esquerda das categorias ind $R$, ind $A$ e ind $C$ e, em relação a algumas classes de álgebras, que podem ser determinadas por essas subcategorias, chegamos ao seguinte enunciado, para um certo tipo de produto fibrado.

Proposição 4. Seja $R$ é o produto fibrado orientado de $A \rightarrow B$ e $C \rightarrow B$. Suponhamos que $\mathcal{B}$ e $\mathcal{A} \cap \mathcal{C}$ sejam subcategorias finitas.

(a) Se A é uma colagem à direita e $C$ é de tipo finito então $R$ é também uma colagem à direita.

(b) Se C é uma colagem à esquerda e A é de tipo finito então $R$ é também uma colagem à esquerda.

(c) Se A é uma colagem à direita e $C$ é uma colagem à esquerda então $R$ é laura.

Um outro assunto estudado foi a dimensão de representação do produto fibrado. A dimensão de representação de uma álgebra de Artin foi introduzida por Auslander em [Aus71]. Ele acreditava que essa invariante poderia dar uma medida de quão longe está uma álgebra de ser de tipo finito. Lembremos que uma álgebra é dita de tipo finito quando possui apenas um número finito de classes de isomorfismos de módulos indecomponíveis. Auslander mostrou que uma álgebra $\Lambda$ não semisimples é de tipo finito se, e somente se, a dimensão de representação rep.dim $\Lambda$ é 2. Iyama [Iya03] provou que a dimensão de representação é sempre finita e Rouquier [Rou06] construiu exemplos de álgebras com rep.dim $\Lambda=r$ para todo $r \geq 1$.

Igusa e Todorov deram, em [IT05], uma conexão interessante com a conjectura finitística. Eles provaram que, se $\Lambda$ tem dimensão de representação no máximo 3 então a dimensão finitística de $\Lambda$ é finita, isto é, o $\sup \{\operatorname{dp} M \mid \operatorname{dp} M<\infty\}$ é finito.

Já conhecemos que a dimensão de representação é no máximo três para diversas classes de álgebras: hereditária [Aus71], inclinada e laura [APT06], colagens à direita e à esquerda [CP04], quase inclinada [Opp10], entre outras. Podemos citar [Rin08] para uma breve descrição do estado atual da pesquisa de dimensão de representação.

Com a tentativa de calcular a dimensão de representação do produto fibrado orientado $R$ a partir das dimensões de representação das álgebras $A$ e $C$ notou-se a importância de, no caso Nakayama orientado, a categoria dos módulos indecomponíveis ind $R$ possuir uma trisecção bem determinada pelas categorias ind $A$, ind $B$ e ind $C$. Com isso em mente iniciamos o estudo de dimensão de representação de uma álgebra de Artin, não necessariamente um produto fibrado, cuja categoria dos módulos indecomponíveis possui uma trisecção com certas condições. Como resultado conseguimos dois dos principais teoremas desse trabalho. Para definição de subcategorias covariantemente finita e contravariantemente finita sugeri$\operatorname{mos}[\mathrm{AS} 80]$. 
Teorema 5. Seja $\Lambda$ uma álgebra de Artin de tipo infinito e $(\mathcal{A}, \mathcal{B}, \mathcal{C})$ uma trisecção de ind $\Lambda$ com $\mathcal{B}$ finita. $S e \mathcal{C} \subseteq \mathcal{R}_{\Lambda}$ e addC é covariantemente finita então

$$
\text { rep.dim } \Lambda=\max \left\{3, \operatorname{rep} \cdot \operatorname{dim}_{\mathcal{A}} \Lambda\right\}
$$

Teorema 6. Seja $\Lambda$ uma álgebra de $\operatorname{Artin} \operatorname{com}(\mathcal{A}, \mathcal{B}, \mathcal{C})$ uma trisecção de ind $\Lambda$. Se $(\mathcal{A} \cup$ $\left.\operatorname{ind} \Lambda_{\mathcal{C}}\right)^{c}$ é finita e ind $\Lambda_{\mathcal{C}}$ é fechada para sucessores Então,

$$
\text { rep.dim } \Lambda \leq \max \left\{\text { rep. } \operatorname{dim}{ }_{\mathcal{A}} \Lambda, \operatorname{rep} \cdot \operatorname{dim} \Lambda_{\mathcal{C}}\right\}
$$

Como principal aplicação desses resultados conseguimos mostrar que a dimensão de representação de álgebras do tipo ada é no máximo 3 (Teorema 5.5.3). E, é claro, para alguns tipos de produto fibrado orientado $R$, dos epimorfismos $A \rightarrow B$ e $C \rightarrow B$, provamos que rep. $\operatorname{dim} R$ não ultrapassa as dimensões de representação das álgebras $A$ e $C$.

\section{Organização do Trabalho}

O primeiro capítulo, chamado de Preliminares, traz um apanhado de definições e notações comuns em Teoria de Representações de álgebras de Artin e, em particular, de álgebras de dimensão finita sobre um corpo, tais como aljavas, representaçoẽs de módulos e aljavas de Auslander-Reiten. Além disso, como um dos objetivos do trabalho é relacionar classes de álgebras em um produto fibrado, esse capítulo ainda contém uma seção com as classes de álgebras citadas no decorrer do trabalho, listando as definições e alguns resultados que são uteis nos demais capítulos. Ainda nesse capítulo, há uma seção que destaca algumas subcategorias especiais da categoria de módulos indecomponíveis de uma álgebra de Artin, tais como as partes direita e esquerda da categoria de módulos, que são úteis na caracterização das classes utilizadas.

O Capítulo 2 é dedicado ao Teorema 2, onde incluímos uma lista de todas as aljavas de tipo Dynkin com um único poço.

O terceiro e o quarto capítulos são dedicados ao Produto fibrado de álgebras de dimensão finita sobre um corpo algebricamente fechado. Começamos o Capítulo $3 \mathrm{com}$ propriedades gerais e, em seguida, tratamos do caso de nosso interesse que é quando as álgebras podem ser determinadas por aljavas com relações. Nesse caso, a aljava ordinária do produto fibrado fica bem determinada a partir das aljavas ordinárias das outras álgebras envolvidas. A partir da Seção 3.2, damos início ao estudo do produto fibrado orientado $R$ de morfismos $A \rightarrow B$ e $C \rightarrow B$, para o qual os módulos projetivos e injetivos sobre $R$ são módulos projetivos e injetivos de $A$ e de $C$ e, com isso, temos uma boa relação entre as dimensões projetiva, injetiva e global dessas álgebras. Mostramos que, para esse tipo de produto fibrado, todo $R$-módulo pode ser visto como um produto fibrado de um $A$-módulo com um $C$-módulo. 
Um caso particular de produto fibrado orientado é o produto fibrado Dynkin orientado que definimos na Seção 3.3.1, e na qual mostramos o Teorema 1.

No Capítulo 4 ainda tratamos do produto fibrado orientado, mas agora estudando suas propriedades através de subcategorias $\mathcal{A}$ e $\mathcal{C}$ de ind $R$ definidas, respectivamente, como sendo os predecessores de certos $A$-módulos injetivos e os sucessores de certos $C$-módulos projetivos. $\mathrm{Na}$ primeira seção, mostramos que a partir de um certo tipo de trisecção da categoria de módulos de uma certa álgebra $\Lambda$ é possível definir epimorfismos de álgebras $A \rightarrow B$ e $C \rightarrow B$ tais que $\Lambda$ é o produto fibrado orientado desses morfismos. Em particular, uma álgebra $a d a$ pode ser vista como um produto fibrado orientado de álgebras associadas as partes direita e esquerda da categoria de módulos. Ainda, com o estudo das categorias $\mathcal{A}$ e $\mathcal{C}$, mostramos, na Seção 4.3, o Teorema 3. Na Seção 4.4, mostramos uma relação entre as partes direita e esquerda das álgebras envolvidas no produto fibrado orientado e, a partir disso, chegamos à Proposição 4. Ainda nessa seção, chegamos a uma generalização de [Lev08](2.4.7) que diz que o produto fibrado é uma álgebra inclinada quando $A$ e $C$ são hereditárias.

No quinto e último capítulo desse trabalho, que é dedicado ao estudo de dimensão de representação, as álgebras consideradas são álgebras de Artin. Começamos com a definição de resolução de aproximação que nós dá uma técnica para calcular a dimensão de representação de uma álgebra de Artin. As seções 5.3 e 5.4, dedicam-se a mostrar que se pudermos dividir a categoria de módulos em pedaços com certas propriedades é possível obter a dimensão de representação da álgebra a partir do cálculo dessa invariante em álgebras menores associadas a esses pedaços. Com isso, chegamos aos Teoremas 5 e 6 . Como consequência desses resultados, na Seção 5.5, damos uma nova demonstração de que a dimesão de representação de uma álgebra laura é no máximo 3 e mostramos que, para as álgebras ada, a dimensão de representação também é no máximo 3. Na última seção desse capítulo, apresentamos algumas aplicações dos resultados sobre dimensão de representação aos produtos fibrados estudados nos primeiros capítulos.

Finalmente, incluímos no Apêndice A um esquema da aljava de Auslander-Reiten da álgebra que aparece nos exemplos 5.3.1, 5.3.3 e 5.4.11. 


\section{Capítulo 1}

\section{Preliminares}

Nesse trabalho trataremos de álgebras de Artin, básicas e conexas e em muitos casos nos restringiremos às álgebras de dimensão finita sobre um corpo algebricamente fechado. Esse primeiro capítulo será dedicado a algumas definições e alguns resultados que serão livremente utilizados no decorrer do trabalho. Para mais detalhes sobre Representações de álgebras de Artin sugerimos, por exemplo, [Ass97, ARS95, ASS06, Coe92].

Em todo o texto, caso não se faça menção contrária, as álgebras consideradas são associativas e com unidade e os módulos são finitamente gerados à direita.

Lembremos que uma $k$-álgebra $\Lambda$ é dita uma álgebra de Artin se $k$ for um anel artiniano e $\Lambda$ for um $k$-módulo finitamente gerado. Para uma álgebra de Artin $\Lambda$, básica e conexa, consideremos $\left\{e_{1}, e_{2}, \ldots, e_{n}\right\}$ um sistema completo de idempotentes ortogonais e primitivos e, neste caso, a unidade de $\Lambda$ é $1_{\Lambda}=\sum_{i=1}^{n} e_{i}$.

Denotaremos por $P(i), I(i)$ e $S(i)$ o projetivo, o injetivo e o simples associados ao idempotente $e_{i}$, respectivamente.

Para uma álgebra de $\operatorname{Artin} \Lambda$ denotaremos $\operatorname{por} \bmod \Lambda$ a categoria cujos objetos são os $\Lambda$-módulos finitamente gerados e por ind $\Lambda$ a subcategoria plena de $\bmod \Lambda$ cujos objetos são um representante de cada classe de isomorfismo dos $\Lambda$-módulos indecomponíveis. Dizemos que a álgebra $\Lambda$ é de tipo (de representação) finito se a categoria ind $\Lambda$ é finita. Caso contrário, $\Lambda$ é dita de tipo infinito. Dada uma categoria $\mathcal{C}$ escreveremos muitas vezes $M \in \mathcal{C}$ para dizer que $M$ é um objeto da categoria $\mathcal{C}$.

Seja $\Lambda$ uma $k$-álgebra de Artin, denotamos por $D$ a dualidade $\operatorname{de} \bmod \Lambda$ em $\bmod \Lambda^{\mathrm{op}}$, dada por $D M=\operatorname{Hom}_{k}(M, k)$.

\subsection{Aljavas e Representações}

Uma aljava é uma quádrupla $\left(Q_{0}, Q_{1}, s, e\right)$ onde $Q_{0}$ e $Q_{1}$ são conjuntos e $s, e: Q_{1} \rightarrow Q_{0}$ são funções. Os elementos de $Q_{0}$ são chamados de vértices de $Q$ e os elementos de $Q_{1}$ são chamados de flechas de $Q$. Dada uma flecha $\alpha$ em $Q_{1}$, chamamos o elemento $s(\alpha)$ de vértice inicial de $\alpha$ e o elemento $e(\alpha)$ de vértice final de $\alpha$. Uma aljava $Q$ é dita finita se os conjuntos $Q_{0}$ e $Q_{1}$ são finitos.

Podemos representar uma aljava por um diagrama como no exemplo a seguir. 


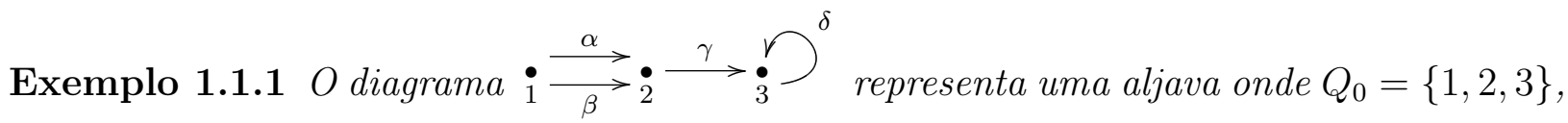
$Q_{1}=\{\alpha, \beta, \gamma, \delta\}, s(\alpha)=s(\beta)=1, s(\gamma)=e(\alpha)=e(\beta)=2$ e $s(\delta)=e(\delta)=e(\gamma)=3$.

Uma subaljava de $Q$ é uma aljava $\left(\tilde{Q}_{0}, \tilde{Q}_{1}, \tilde{s}, \tilde{e}\right)$ de forma que $\tilde{Q}_{0} \subseteq Q_{0}, \tilde{Q}_{1} \subseteq Q_{1}$, $\tilde{s}=\left.s\right|_{\tilde{Q}_{1}}$ e $\tilde{e}=\left.e\right|_{\tilde{Q}_{1}}$. Uma subaljava é dita plena se a flecha $a \stackrel{\alpha}{\rightarrow} b$ de $Q_{1}$ estiver em $\tilde{Q}_{1}$ sempre que $a, b \in \tilde{Q}_{0}$.

Um caminho $\omega$ em $Q$ de comprimento $n>0$ é uma sequência de flechas $\omega=\alpha_{1} \cdots \alpha_{n}$, tal que $e\left(\alpha_{i}\right)=s\left(\alpha_{i+1}\right)$ para $1 \leq i<n$. Por convenção, um caminho de comprimento zero (ou caminho trivial) é um caminho sem flechas associado a um vértice $a \in Q_{0}$, que denotamos por $e_{a}$. Para um caminho não trivial $\omega=\alpha_{1} \cdots \alpha_{n}$ definimos o vértice inicial de $\omega$ por $s(\omega):=s\left(\alpha_{1}\right)$ e o vértice final de $\omega$ por $e(\omega):=e\left(\alpha_{n}\right)$. Para um caminho trivial $e_{a}$ definimos $s\left(e_{a}\right)=e\left(e_{a}\right)=a$. Um caminho $\omega$ de comprimento $n \geq 1$ é dito um ciclo orientado quando $s(\omega)=e(\omega)$. Uma subaljava $\tilde{Q}$ é dita convexa se dado um caminho $\omega=\alpha_{1} \cdots \alpha_{n}$ em $Q$ tal que $s(\omega)$ e $e(\omega)$ estão em $\tilde{Q}_{0}$ então cada $s\left(\alpha_{i}\right)$ e cada $e\left(\alpha_{i}\right)$ também estão em $\tilde{Q}_{0}$.

Para cada flecha $\alpha: a \rightarrow b$, associamos um inverso formal $\alpha^{-1}: b \rightarrow a \operatorname{com} s\left(\alpha^{-1}\right)=e(\alpha)$ e $e\left(\alpha^{-1}\right)=s(\alpha)$. Um passeio de comprimento $n \geq 1$ de $a \in Q_{0}$ para $b \in Q_{0}$ é uma sequência $\gamma=\alpha_{1}^{\epsilon_{1}} \alpha_{2}^{\epsilon_{2}} \cdots \alpha_{n}^{\epsilon_{n}} \operatorname{com} \epsilon_{i} \in\{-1,1\}, s\left(\alpha_{1}^{\epsilon_{1}}\right)=a, e\left(\alpha_{n}^{\epsilon_{n}}\right)=b$ e $e\left(\alpha_{i}^{\epsilon_{i}}\right)=s\left(\alpha_{i+1}^{\epsilon_{i+1}}\right)$ para cada $1 \leq i \leq n$.

Dado um vértice $a \in Q_{0}$, a subaljava plena $Q_{a}$ de $Q$ formada pelos vértices $b \in Q_{0}$ tais que existe um passeio de $a$ para $b$ é chamada de componente conexa de $Q$ contendo $a$. Quando $Q=Q_{a}$ para algum a dizemos que a aljava $Q$ é conexa.

Dadas duas aljavas $\left(Q_{0}, Q_{1}, s, e\right)$ e $\left(Q_{0}^{\prime}, Q_{1}^{\prime}, s^{\prime}, e^{\prime}\right)$ um morfismo de aljavas é uma função $\Phi: Q_{0} \cup Q_{1} \rightarrow Q_{0}^{\prime} \cup Q_{1}^{\prime} \operatorname{com} \Phi\left(Q_{0}\right) \subseteq Q_{0}^{\prime}$ e $\Phi\left(Q_{1}\right) \subseteq Q_{1}^{\prime}$ tal que $\Phi \circ s=s^{\prime} \circ \Phi$ e $\Phi \circ e=e^{\prime} \circ \Phi$.

\section{Álgebras de caminhos}

Para essa seção, e sempre que nos referirmos a álgebras de caminhos, $k$ denotará um corpo algebricamente fechado.

Consideremos $Q$ uma aljava finita e seja $k Q$ o $k$-espaço vetorial cuja base é o conjunto de todos os caminhos de $Q$. Definimos em $k Q$ o seguinte produto: dados $\gamma$ e $\sigma$ caminhos de $Q$, então

- se $e(\gamma) \neq s(\sigma), \gamma \cdot \sigma=0$

- se $e(\gamma)=s(\sigma), \gamma \cdot \sigma=\left\{\begin{array}{cl}\sigma, & \text { se } \gamma=e_{a} \text { para algum } a \in Q_{0} \\ \gamma, & \text { se } \sigma=e_{a} \text { para algum } a \in Q_{0} \\ \alpha_{1} \cdots \alpha_{n} \beta_{1} \cdots \beta_{t}, & \text { se } \gamma=\alpha_{1} \cdots \alpha_{n} \text { e } \sigma=\beta_{1} \cdots \beta_{t}\end{array}\right.$

Estendendo esse produto por linearidade aos elementos de $k Q$ temos que $k Q$ é uma $k$-álgebra, a qual chamamos de álgebra de caminhos de $Q$. 
Exemplo 1.1.2 Seja $Q$ a aljava 1

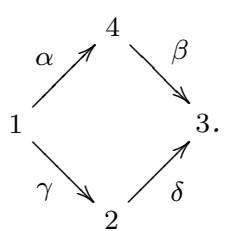

$A$ base de $k Q$ como $k$-espaço vetorial é $\left\{e_{1}, e_{2}, e_{3}, e_{4}, \alpha, \beta, \gamma, \delta, \alpha \beta, \gamma \delta\right\}$ e portanto a dimensão de $k Q$ é $\operatorname{dim}_{k} k Q=10$. Quanto à multiplicação, teremos, por exemplo, $\alpha \cdot \beta=\alpha \beta, \alpha \cdot \delta=0$, $e_{2} \cdot \delta=\delta$, etc.

Denotaremos por $J_{Q}$ o ideal de $k Q$ gerado pelas flechas de $Q$.

Proposição 1.1.3 Sejam $Q$ uma aljava finita com $Q_{0}=\{1, \ldots, n\}, k Q$ sua álgebra de caminhos e $e_{i}$ o caminho trivial associado ao vértice $i \in Q_{0}$. Então:

1. $k Q$ é uma álgebra associativa.

2. o conjunto $\left\{e_{i}\right\}_{i \in Q_{0}}$ é um sistema completo de idempotentes ortogonais primitivos de $k Q$. Em particular, $k Q$ tem identidade $1=e_{1}+\cdots+e_{n}$.

3. $k Q$ é uma álgebra básica e $k Q=e_{1}(k Q) \oplus \cdots \oplus e_{n}(k Q)$ é a decomposição de $k Q$ em módulos indecomponíveis.

4. kQ tem dimensão finita se, e somente se, $Q$ não possui ciclos orientados.

5. kQ é uma álgebra conexa se, e somente se, $Q$ é uma aljava conexa.

6. $J_{Q}=\operatorname{rad} k Q$ se, e somente se, $Q$ não possui ciclos orientados.

7. o número de flechas de i para $j$ é igual ao número $\operatorname{dim}_{k}\left(e_{i}\left(\frac{J_{Q}}{J_{Q}^{2}}\right) e_{j}\right)$.

Um ideal $\mathcal{I}$ de $k Q$ é dito admissível se existir $n \geq 2$ tal que $J_{Q}^{n} \subseteq \mathcal{I} \subseteq J_{Q}^{2}$.

Uma relação $\rho$ em $Q$ é uma combinação linear de caminhos de comprimento pelo menos dois, todos com os mesmos vértices iniciais e finais.

Um ideal admissível $\mathcal{I}$ sempre possui um conjunto finito de geradores formado por relações. Por isso, chamamos o par $(Q, \mathcal{I})$ de aljava com relações.

Proposição 1.1.4 Sejam $Q$ uma aljava finita, $\mathcal{I}$ um ideal admissivel de $k Q$ e $\frac{k Q}{\mathcal{I}}$ a álgebra quociente. Então:

1. $\frac{k Q}{\mathcal{I}}$ tem dimensão finita sobre $k$.

2. o conjunto $\left\{e_{i}+\mathcal{I}\right\}_{i \in Q_{0}}$ é um sistema completo de idempotentes ortogonais primitivos de $\frac{k Q}{\mathcal{I}}$.

3. $\frac{k Q}{\mathcal{I}}$ é uma álgebra básica. 
4. $\frac{k Q}{\mathcal{I}}$ é uma álgebra conexa (ou indecompon/'ivel) se, e somente se, $Q$ é uma aljava conexa.

5. $\operatorname{rad} \frac{k Q}{\mathcal{I}}=\frac{J_{Q}}{\mathcal{I}}$.

Teorema 1.1.5 Seja A uma k-álgebra básica e de dimensão finita sobre $k$ algebricamente fechado. Então existe uma aljava $Q_{A}$ e um epimorfismo $\eta_{A}: k Q_{A} \rightarrow A$ tal que $\mathcal{I}_{A}:=\operatorname{ker} \eta_{A}$ é um ideal admissivel de $k Q_{A}$. Em particular, $A \cong \frac{k Q_{A}}{\mathcal{I}_{\mathcal{A}}}$.

A aljava $Q_{A}$ do teorema acima, que é única, é chamada de aljava ordinária de $A$. Já o epimorfismo, que não é necessáriamente único, é chamado uma apresentação de $A$.

\section{Módulos e representações}

Sejam $k$ um corpo algebricamente fechado e $Q$ uma aljava finita. Uma representação de $Q$ é dada por $V=\left(\left(V_{i}\right)_{i \in Q_{0}},\left(T_{\alpha}\right)_{\alpha \in Q_{1}}\right)$, onde para cada $i \in Q_{0}, V_{i}$ é um $k$-espaço vetorial de dimensão finita e para cada $\alpha \in Q_{1}, T_{\alpha}$ é uma transformação linear de $V_{s(\alpha)}$ em $V_{e(\alpha)}$.

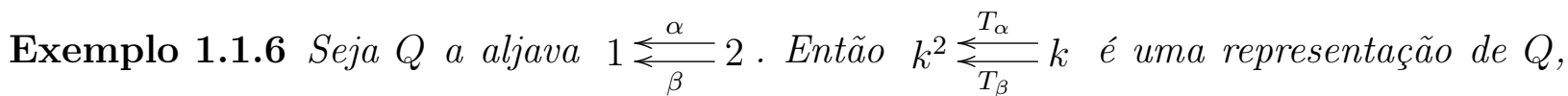
onde $V_{1}=k^{2}, V_{2}=k, T_{\alpha}=\left[\begin{array}{l}1 \\ 0\end{array}\right]$ e $T_{\beta}=\left[\begin{array}{l}0 \\ 1\end{array}\right]$.

Seja $\omega=\alpha_{1} \alpha_{2} \ldots \alpha_{n}$ um caminho não trivial de $Q$. Definimos a transformação linear $T(\omega): V_{s(\omega)} \rightarrow V_{e(\omega)}$ dada pela composta $T_{\alpha_{1}} T_{\alpha_{2}} \cdots T_{\alpha_{n}}$. Estendemos esta definição para uma combinação linear de caminhos $\omega=\sum_{i=1}^{t} \lambda_{i} \omega_{i}$, onde $s\left(\omega_{i}\right)=s\left(\omega_{j}\right)$ e $e\left(\omega_{i}\right)=e\left(\omega_{j}\right)$, $\forall i, j \in\{1, \ldots, n\}$, fazendo $T(\omega)=\sum_{i=1}^{t} \lambda_{i} T\left(\omega_{i}\right)$.

Uma representação de $(Q, \mathcal{I})$ é uma representação de $Q$ de forma que para cada relação $\omega$ de $\mathcal{I}$ tem-se $T(\omega)=0$.

Dadas duas representações $V=\left(\left(V_{i}\right)_{i \in Q_{0}},\left(T_{\alpha}\right)_{\alpha \in Q_{1}}\right)$ e $W=\left(\left(W_{i}\right)_{i \in Q_{0}},\left(S_{\alpha}\right)_{\alpha \in Q_{1}}\right)$, um morfismo $\Phi: V \rightarrow W$ é uma família $\left\{\phi_{i}\right\}_{i \in Q_{0}}$ de transformações lineares tal que, para cada flecha $i \stackrel{\alpha}{\rightarrow} j$ o seguinte diagrama é comutativo

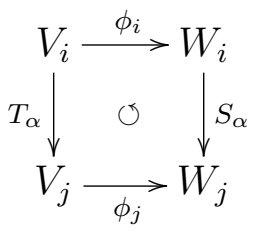


ou seja, $\phi_{j} T_{\alpha}=S_{\alpha} \phi_{i}$. A composta de dois morfismos é definida coordenada a coordenada. Definimos então a categoria $\bmod (Q, \mathcal{I})$ cujos objetos são as representações de $(Q, \mathcal{I})$ e os morfismos são os descritos acima.

Teorema 1.1.7 Seja $A=\frac{k Q}{\mathcal{I}}$ onde $Q$ é uma aljava finita e $\mathcal{I}$ um ideal admissivel de $k Q$. Então as categorias $\bmod (Q, \mathcal{I})$ e $\bmod A$ são equivalentes.

Pelo teorema acima podemos identificar os $A$-módulos com representações de $(Q, \mathcal{I})$. Em especial, as representações que correspondem aos $A$-módulos simples, projetivos indecomponíveis, injetivos indecomponíveis, radical, socle e topo podem ser calculados a partir da aljava ordinária $Q$ e das relações $(\mathcal{I})$. Para detalhes dessas descrições ver, por exemplo, [ASS06, Coe92].

Dado um módulo $M$ cuja representação é $\left(\left(V_{i}\right)_{i \in Q_{0}},\left(T_{\alpha}\right)_{\alpha \in Q_{1}}\right)$ definimos o suporte de $M$, denotado por $\operatorname{supp} M$, como sendo a subaljava de $Q$ definida pelos vértices $x$ tais que $V_{x}$ é não nulo e pelas flechas $\alpha$ tais que $T_{\alpha}$ é não nula. Observe que se $M$ é indecomponível então $\operatorname{supp} M$ é uma aljava conexa.

\subsection{Teoria de Auslander-Reiten}

Dados dois módulos indecomponíveis $M$ e $N$, um morfismo $f: M \rightarrow N$ é dito irredutível se (i) $f$ não é um monomorfismo que cinde e nem um epimorfismo que cinde e (ii) se $f=g h$ então $g$ é um epimorfismo que cinde ou $h$ é um monomorfismo que cinde.

Para uma álgebra de Artin $\Lambda$ associamos uma aljava, que será denotada por $\Gamma_{\Lambda}$ e é conhecida como aljava de Auslander-Reiten, cujo conjunto de vértices é formado pelos objetos de ind $\Lambda$ e as flechas são dadas pelos morfismos irredutíveis. Portanto, se $\Lambda$ é uma álgebra de tipo infinito, então a aljava $\Gamma_{\Lambda}$ tem infinitos vértices e, nesse caso, pode conter infinitas componentes conexas.

\section{Sequências de Auslander-Reiten}

Uma sequência exata curta de $\Lambda$-módulos que não cinde, $0 \rightarrow N \stackrel{f}{\rightarrow} E \stackrel{g}{\rightarrow} M \rightarrow 0$, é uma sequência de Auslander-Reiten se:

(i) $M$ e $N$ são indecomponíveis;

(ii) $\operatorname{dados} X \in \bmod \Lambda$ e $h: X \rightarrow M$ um morfismo que não seja um epimorfismo que cinde, existe $\bar{h}: X \rightarrow E$ tal que $g \bar{h}=h$.

Podemos trocar (ii) pela condição equivalente:

(iii) dados $X \in \bmod \Lambda$ e $h: N \rightarrow X$ um morfismo que não seja um monomorfismo que cinde, existe $\bar{h}: E \rightarrow X$ tal que $\bar{h} f=h$. 
Proposição 1.2.1 (Existência e unicidade) Sejam $\Lambda$ uma álgebra de Artin e $M$ e $N$ dois $\Lambda$-módulos indecomponíveis.

1. Se $M$ não for projetivo, então existe e é única, a menos de isomorfismos, a sequência de Auslander-Reiten terminando em $M$ :

$$
0 \rightarrow N \rightarrow E \rightarrow M \rightarrow 0
$$

Neste caso, denotamos $N=\tau M$.

2. Se $N$ não for injetivo, então existe e é única, a menos de isomorfismos, a sequência de Auslander-Reiten começando em $N$ :

$$
0 \rightarrow N \rightarrow E \rightarrow M \rightarrow 0
$$

Neste caso, denotamos $M=\tau^{-1} N$.

Na verdade $\tau$ e $\tau^{-1}$ são funtores conhecidos como transladados de Auslander-Reiten. Podemos escrever uma sequência de Auslander-Reiten da seguinte forma

$$
0 \rightarrow N \stackrel{f}{\rightarrow} \bigoplus_{i=1}^{n} E_{i}^{t_{i}} \stackrel{g}{\rightarrow} M \rightarrow 0
$$

com cada $E_{i}$ indecomponível, $f=\left[\begin{array}{c}f_{1} \\ \vdots \\ f_{n}\end{array}\right]$ onde $f_{i}=\left[\begin{array}{c}f_{i 1} \\ \vdots \\ f_{i t_{i}}\end{array}\right]: N \rightarrow E_{i}^{t_{i}}$ e $g=\left[\begin{array}{lll}g_{1} & \cdots & g_{n}\end{array}\right]$ onde $g_{i}:\left[\begin{array}{lll}g_{i 1} & \cdots & g_{i t_{i}}\end{array}\right]: E_{i}^{t_{i}} \rightarrow M$. Nesse caso, cada $f_{i j}$ e cada $g_{i j}$ é um morfismo irredutível.

Se $M$ é um $\Lambda$-módulo indecomponível e não projetivo, então o número de flechas que saem de $[\tau M]$ é igual ao número de flechas que chegam em $[M]$. Por outro lado, se $M$ é um $\Lambda$-módulo indecomponível e não injetivo então o número de flechas que saem de $[M]$ é igual ao número de flechas que chegam em $\left[\tau^{-1} M\right]$.

$\mathrm{Na}$ aljava de Auslander-Reiten tal sequência corresponderia a um diagrama da forma:

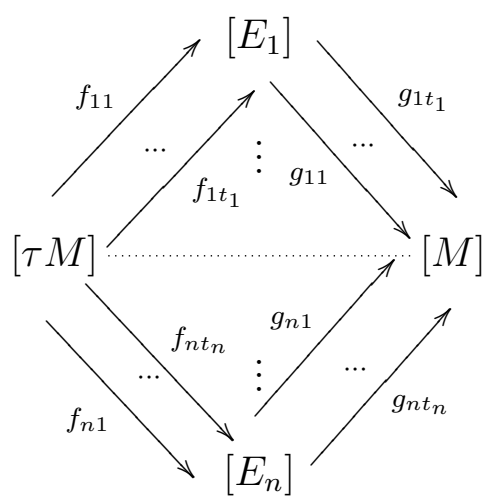


Exemplo 1.2.2 Sejam $k$ um corpo algebricamente fechado, $Q$ a aljava $1 \stackrel{\alpha}{\leftarrow} 2 \stackrel{\beta}{\leftarrow} 3$ e $\mathcal{I}$ o ideal de $k Q$ gerado por $\beta \alpha$. A aljava de Auslander-Reiten de $\frac{k Q}{\mathcal{I}}$ tem a seguinte forma:

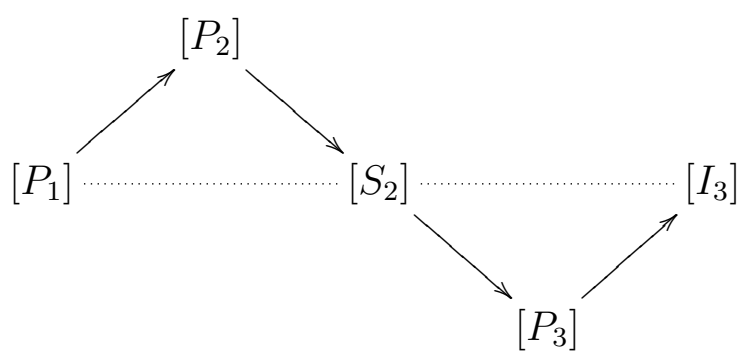

\subsection{Subcategorias especiais}

Por todo o trabalho utilizaremos algumas subcategorias de $\bmod \Lambda$ e de ind $\Lambda$. Essa seção trata de fixar notações e algumas propriedades de tais categorias.

Seja $\mathcal{C}$ uma subcategoria $\operatorname{de} \bmod \Lambda$. Denotamos por add $\mathcal{C}$ a subcategoria plena de $\bmod \Lambda$ cujos objetos são somas diretas de somandos diretos que estão em $\mathcal{C}$. Para um $\Lambda$-módulo $M$ denotaremos add $\{M\}$ por add $M$. Denotamos por Gen $M$ a subcategoria de $\bmod \Lambda$ cujos objetos são os módulos $X$ tais que existe um epimorfismo $M_{0} \rightarrow X \operatorname{com} M_{0}$ em add $M$. Dualmente, denotamos por $\operatorname{Cogen} M$ a subcategoria de $\bmod \Lambda$ cujos objetos são os módulos $X$ tais que existe um monomorfismo $X \rightarrow M_{0}$ com $M_{0}$ em add $M$. Observe que Gen $\Lambda=$ $\bmod \Lambda=\operatorname{Cogen} D \Lambda$.

Dados dois $\Lambda$-módulos indecomponíveis $M$ e $N$, um caminho de $M$ para $N$, denotado por $M \rightsquigarrow N$, é uma sequência de morfismos não nulos

$$
M=X_{0} \rightarrow X_{1} \rightarrow \cdots \rightarrow X_{t-1} \rightarrow X_{t}=N
$$

onde $t \geq 1$ e cada $X_{i}$ está em ind $\Lambda$. Neste caso, dizemos que $M$ é um predecessor de $N$ e que $N$ é um sucessor de $M$. Dado $M$ em $\bmod \Lambda$ denotamos por $\operatorname{Pred} M$ a subcategoria plena de ind $\Lambda$ cujos objetos são os predecessores de algum somando indecomponível de $M$. Dualmente, definimos Succ $M$ para os sucessores dos somandos de $M$. Uma subcategoria $\mathcal{C}$ de ind $\Lambda$ é dita fechada para predecessores se, sempre que existir um caminho $M \rightsquigarrow N$ com $M$ em $\mathcal{C}$ então $N$ também está em $\mathcal{C}$. Dualmente, $\mathcal{C}$ é dita fechada para sucessores se, sempre que existir um caminho de $M$ para $N \operatorname{com} N$ em $\mathcal{C}$ então $M$ também está em $\mathcal{C}$.

\section{Partes direita e esquerda}

Denotemos por $\operatorname{dp} M$ a dimensão projetiva do módulo $M$ e por $\operatorname{di} M$ a dimensão injetiva de $M$.

As partes direita e esquerda da categoria de módulos de uma álgebra $\Lambda$ são definidas como as subcategorias plenas de ind $\Lambda$ dadas, respectivamente, por 


$$
\mathcal{R}_{\Lambda}=\{M \in \operatorname{ind} \Lambda \mid \operatorname{di} N \leq 1 \text { para cada sucessor } N \text { de } M\}
$$

$\mathrm{e}$

$$
\mathcal{L}_{\Lambda}=\{M \in \operatorname{ind} \Lambda \mid \operatorname{dp} N \leq 1 \text { para cada predecessor } N \text { de } M\} .
$$

É fácil ver que a parte direita $\mathcal{R}_{\Lambda}$ é fechada para sucessores e que a parte esquerda $\mathcal{L}_{\Lambda}$ é fechada para predecessores.

\subsection{Algumas classes de álgebras}

Algumas classes de álgebras serão constantemente citadas nesse trabalho, por isso faremos aqui um pequeno apanhado de suas definições contemplando algumas equivalências e alguns resultados. Note que elas podem ser caracterizadas através de propriedades das partes direita e esquerda da categoria de módulos. Para mais informações sobre algumas das álgebras aqui citadas sugerimos $\left[\mathrm{ACL}^{+} 05\right]$.

\section{Álgebras hereditárias}

Uma álgebra de Artin $\Lambda$ é dita hereditária quando todo ideal de $\Lambda$ é um $\Lambda$-módulo projetivo. O teorema a seguir nos dá outras formas equivalentes para essa definição. Para mais detalhes ver, por exemplo, [ASS06] (VII.1).

Teorema 1.4.1 Para uma álgebra de artin $\Lambda$ são equivalentes:

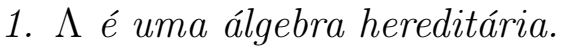

2. A dimensão global de $\Lambda$, dim.gl $\Lambda$, é no máximo 1 .

3. Todo submódulo de uma $\Lambda$-módulo projetivo é projetivo.

4. Todo quociente de um $\Lambda$-módulo injetivo é injetivo.

Como a dimensão global de uma álgebra hereditária é no máximo 1, é fácil ver também que uma álgebra $\Lambda$ é hereditária se, e somente se, a categoria dos módulos indecomponíveis $\operatorname{ind} \Lambda$ coincide com a parte esquerda $\mathcal{L}_{\Lambda}$, ou equivalentemente, com a parte direita $\mathcal{R}_{\Lambda}$.

No caso de álgebras de dimensão finita sobre um corpo $k$ algebricamente fechado, as álgebras hereditárias $\Lambda$ são aquelas tais que o ideal admissível $I$ com $\Lambda \cong k Q / I$ é nulo, ou seja, são justamente as álgebras de caminho da forma $k Q$ onde $Q$ é uma aljava sem ciclos. 


\section{Álgebras inclinadas}

Para teoria de inclinação sugerimos [HR82], [Ass90] e [ACPV08].

Lembremos que um $\Lambda$-módulo $T$ é dito inclinante se ele satisfaz as três condições seguintes: (i) a dimensão projetiva de $T$ é no máximo 1; (ii) $\operatorname{Ext}^{1}(T, T)=0$ e (iii) o número de somandos indecomponíveis de $T$ é igual ao número de $\Lambda$-módulos simples. Se $H$ é uma álgebra hereditária e $T$ é um $H$-módulo inclinante então a álgebra $\operatorname{End}_{H} T$ é chamada de álgebra inclinada. Equivalentemente, $\Lambda$ é inclinada se existir um módulo inclinante $T$ tal que a álgebra de endomorfismos $\operatorname{End}_{\Lambda} T$ é hereditária.

\section{Álgebras quase inclinadas}

Em [HRS96], Happel-Reiten-Smalø, generalizaram a noção de álgebra inclinada, definindo as quase inclinadas. A definição original era outra mas elas podem ser caracterizadas da seguinte maneira. Uma álgebra $\Lambda$ é quase inclinada se (i) a dimensão global é no máximo 2 e (ii) para cada $\Lambda$-módulo $M$ tem-se que a dimensão projetiva de $M$ é no máximo 1 ou a dimensão injetiva é no máximo 1.

É bem conhecido que uma álgebra inclinada satisfaz essas duas condições e portanto, como o nome sugere, a classe das álgebras quase inclinadas contém a classe das inclinadas.

Para uma álgebra quase inclinada tem-se a seguinte trisecção na categoria de módulos indecomponíveis:

$$
\left(\mathcal{L}_{\Lambda} \backslash \mathcal{R}_{\Lambda}, \mathcal{L}_{\Lambda} \cap \mathcal{R}_{\Lambda}, \mathcal{R}_{\Lambda} \backslash \mathcal{L}_{\Lambda}\right)
$$

ou seja,

$\operatorname{Hom}_{\Lambda}\left(\mathcal{L}_{\Lambda} \cap \mathcal{R}_{\Lambda}, \mathcal{L}_{\Lambda} \backslash \mathcal{R}_{\Lambda}\right)=0, \operatorname{Hom}_{\Lambda}\left(\mathcal{R}_{\Lambda} \backslash \mathcal{L}_{\Lambda}, \mathcal{L}_{\Lambda} \cap \mathcal{R}_{\Lambda}\right)=0$ e $\operatorname{Hom}_{\Lambda}\left(\mathcal{R}_{\Lambda} \backslash \mathcal{L}_{\Lambda}, \mathcal{L}_{\Lambda} \backslash \mathcal{R}_{\Lambda}\right)=0$

Além disso, $\Lambda$ é quase inclinada se, e somente se, a parte esquerda $\mathcal{L}_{\Lambda}$ contém todos os $\Lambda$-módulos projetivos indecomponíveis.

Um resultado que nos será útil é o seguinte.

Teorema 1.4.2 ( [HRS96] (II. 3.4)) Para uma álgebra quase inclinada $\Lambda$ são equivalentes:

1. $\Lambda$ é uma álgebra inclinada.

2. $\mathcal{R}_{\Lambda}$ contém um $\Lambda$-módulo projetivo.

3. $\mathcal{L}_{\Lambda}$ contém um $\Lambda$-módulo injetivo.

\section{Álgebras shod}

Em [CL99], Coelho-Lanzilotta introduziram o conceito de álgebras shod, que engloba a classe das álgebras quase inclinadas. 
Uma álgebra $\Lambda$ é dita shod se cada $\Lambda$-módulo indecomponível $M$ tem dimensão projetiva no máximo 1 ou dimensão injetiva no máximo 1. Equivalentemente, a álgebra $\Lambda$ é shod se $\operatorname{ind} \Lambda=\mathcal{L}_{\Lambda} \cup \mathcal{R}_{\Lambda}$.

Temos então, que uma álgebra quase inclinada é uma álgebra shod com dimensão global 2. Uma álgebra shod que não é quase inclinada é chamada de shod estrita.

\section{Álgebras colagens}

Em [AC94], Assem-Coelho introduziram o conceito de colagens laterais de álgebras inclinadas. Estas são álgebras cujas aljavas ordinárias podem ser vistas como colagens de uma aljava ordinária de uma álgebra inclinada com uma aljava ordinária de uma álgebra de tipo finito. Utilizando as partes direita e esquerda podemos definir tais álgebras como segue: $\Lambda$ é uma colagem à direita se $\mathcal{L}_{\Lambda}$ é cofinita em ind $\Lambda$ e $\Lambda$ é uma colagem à esquerda se $\mathcal{R}_{A}$ é cofinita em ind $\Lambda$.

\section{Álgebras laura}

Em [AC03], Assem-Coelho introduziram o conceito de álgebras colagens bilaterais de álgebras inclinadas, também conhecidas como álgebras laura, que podem ser definidas como sendo àquelas em que a união $\mathcal{L}_{\Lambda} \cup \mathcal{R}_{\Lambda}$ é cofinita em ind $\Lambda$.

Observe que esta classe de álgebras contém as álgebras shod e consequentemente as quase inclinadas e inclinadas. É fácil ver também que as colagens à direita ou à esquerda também são lauras.

\section{Álgebras ada}

As álgebras ada foram introduzidas recentemente em [ACLV12]. Uma álgebra $\Lambda$ é dita ada se todos os projetivos indecomponíveis e todos os injetivos indecomponíveis estão na união $\mathcal{L}_{\Lambda} \cup \mathcal{R}_{\Lambda}$. A álgebra $\Lambda$ é dita ada estrita se for ada mas não for quase inclinada. 


\section{Capítulo 2}

\section{Dynkin com um único poço}

Para a álgebra $k Q$, onde $Q$ é uma aljava da forma $1 \longleftarrow 2<\cdots<n-1 \longleftarrow n$, é conhecido o fato seguinte, utilizado por exemplo em [IPTZ87] $(3.5)$ : se $\left(\left(V_{i}\right)_{i \in Q_{0}},\left(f_{\alpha}\right)_{\alpha \in Q_{1}}\right)$ é uma representação do $k Q$-módulo indecomponível $M$ e cada $f_{\alpha}$ é uma sobrejeção então $M$ é um $k Q$-módulo injetivo.

O objetivo desse capítulo é mostrar que esse fato também vale para uma álgebra $k Q$ com $Q$ sendo uma aljava de tipo Dynkin com um único poço. Observe que isso inclui o caso citado acima que trata de uma aljava de tipo $\mathbb{A}_{n}$ linearmente orientada. No próximo capítulo, esse resultado será de suma importância para uma generalização de [IPTZ87](3.5).

Começaremos com uma lista com todas as aljavas de tipo Dynkin com um único poço. Em seguida, lembraremos a definição de forma quadrática associada a uma aljava e alguns resultados que serão utilizados para a demontração do resultado enunciado.

\subsection{Lista das aljavas de tipo Dynkin com um único poço}

Nessa seção faremos uma lista das aljavas de tipo Dynkin que possuem um único poço. Tipo $\mathbb{A}_{n}$

1.

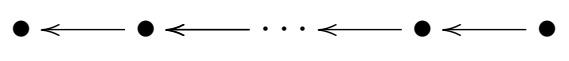

Tipo $\mathbb{D}_{n}$

1.

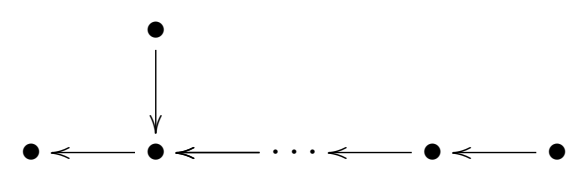

2 .

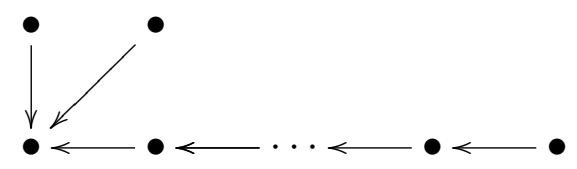

2.

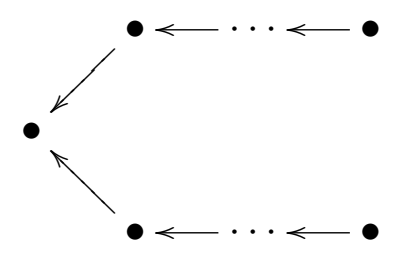

3.

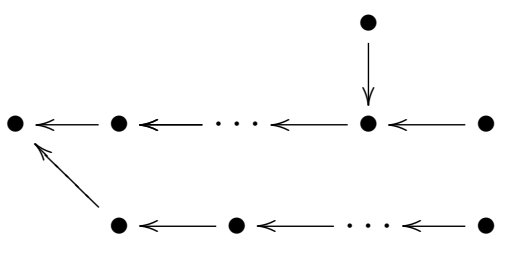

4.

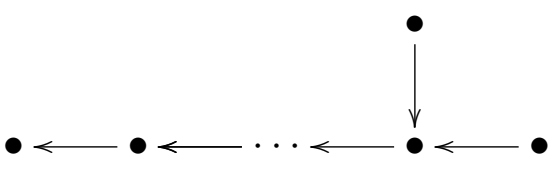


Tipo $\mathbb{E}_{6}$

1.
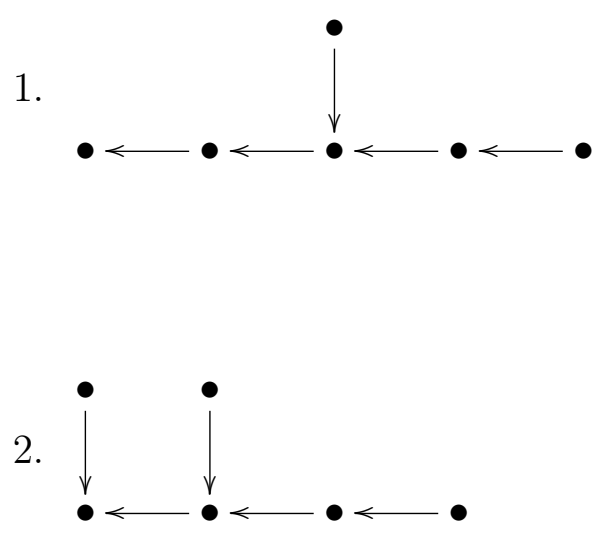

Tipo $\mathbb{E}_{7}$

1.

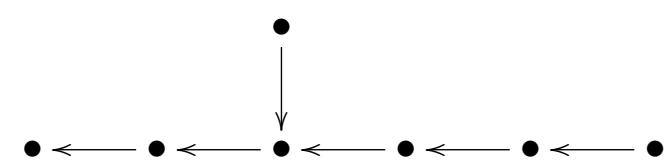
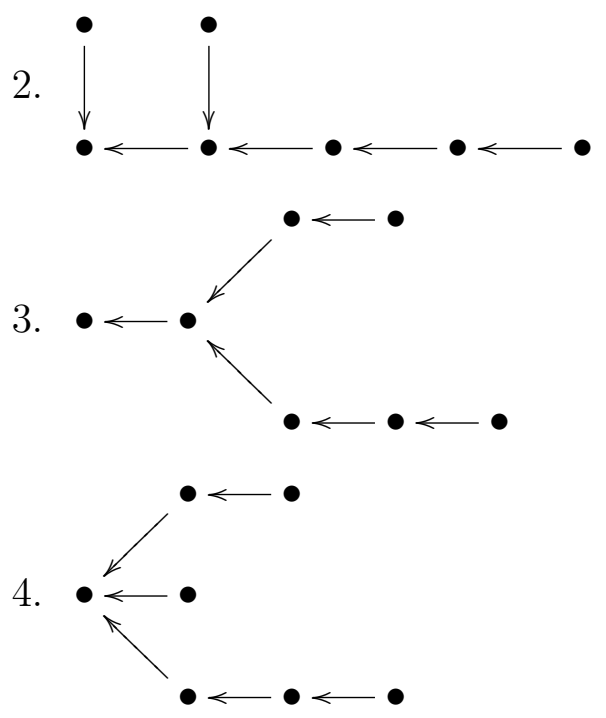

Tipo $\mathbb{E}_{8}$
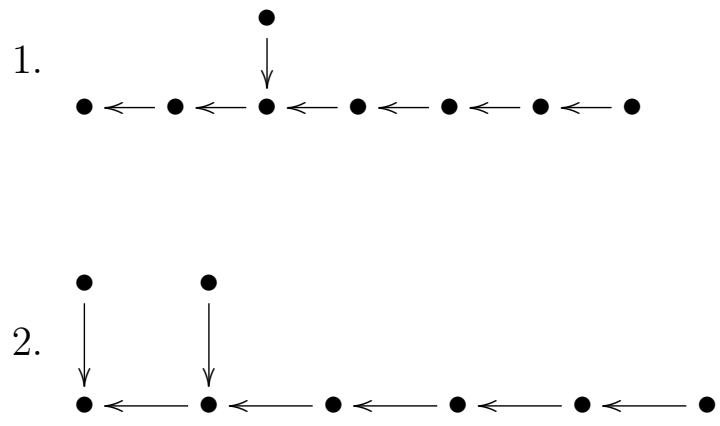

3.

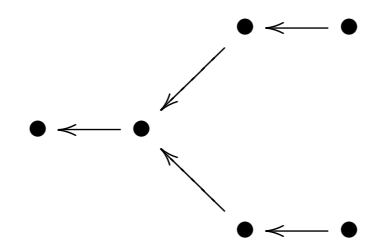

4.

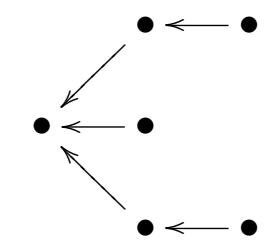

5.

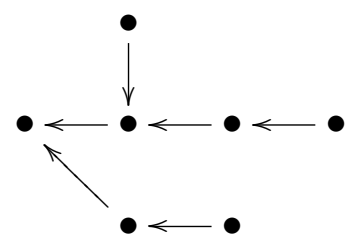

6.

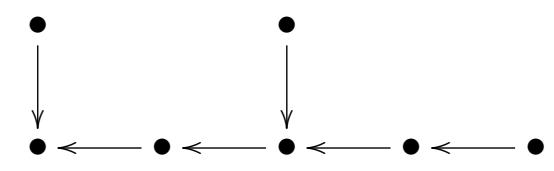

7.

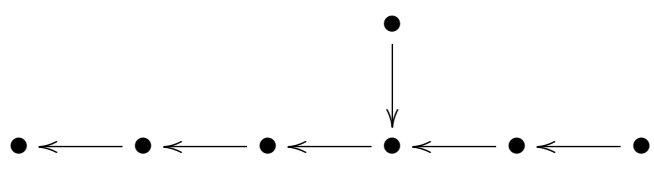

3.

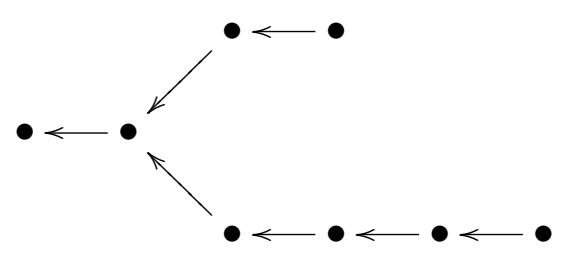


4.

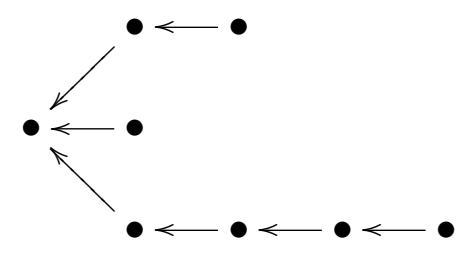

5.

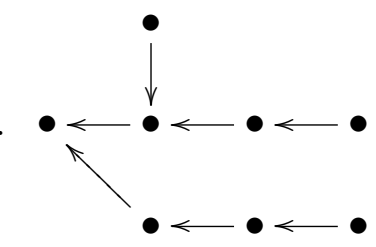

6.

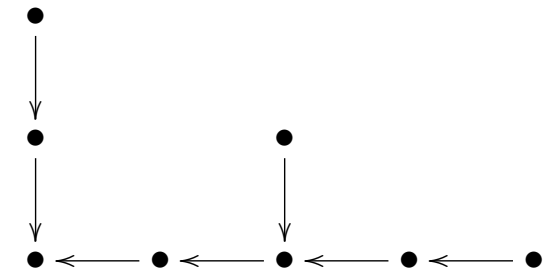

7 .

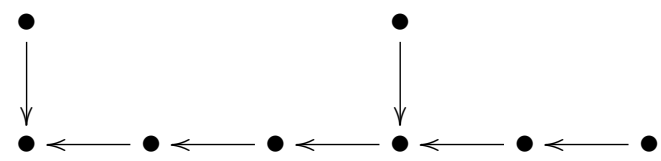

8.

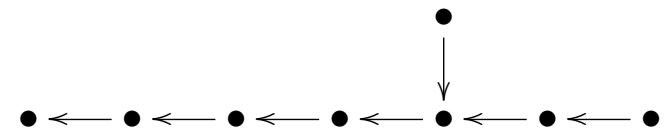

\subsection{Dynkin com um único poço e módulos injetivos}

Seja $Q$ uma aljava finita, conexa e acíclica e $n$ o número de vértices de $Q$. A forma quadrática da aljava $Q$ é definida por

$$
q_{Q}\left(x_{1}, \ldots, x_{n}\right)=\sum_{i \in Q_{0}} x_{i}^{2}-\sum_{\alpha \in Q_{1}} x_{s(\alpha)} x_{e(\alpha)}
$$

Note que essa definição não depende da orientação da aljava $Q$.

Dizemos que o vetor $\left(x_{1}, x_{2}, \ldots, x_{n}\right) \in \mathbb{Z}^{n}$ é uma raiz da forma quadrática $q_{Q}$ quando $q_{Q}\left(x_{1}, x_{2}, \ldots, x_{n}\right)=1$. Tal raiz é dita positiva se $x_{i} \geq 0$ para cada $i \in\{1,2, \ldots, n\}$. No caso em que $Q$ é de tipo Dynkin, existe uma bijeção entre os módulos indecomponíveis em indkQ e as raízes positivas de $q_{Q}$. Tal bijeção é dada por $M \mapsto \underline{\operatorname{dim}} M$ (Ver [ASS06](VII.5.10)).

Se $\left(\left(V_{i}\right)_{i \in Q_{0}},\left(f_{\alpha}\right)_{\alpha \in Q_{1}}\right)$ é uma representação do $k Q$-módulo indecomponível $M$ então o vetor dimensão de $M$ é $\underline{\operatorname{dim}} M=\left(\operatorname{dim}_{k} V_{1}, \operatorname{dim}_{k} V_{2}, \ldots, \operatorname{dim}_{k} V_{n}\right)$.

Observe que, portanto, se $M, N \in \operatorname{ind} k Q$ são módulos tais que $\underline{\operatorname{dim}} M=\underline{\operatorname{dim}} N$ então $M \cong N$.

Lembremos também como se pode construir a representação $\left(\left(V_{i}\right)_{i \in Q_{0}},\left(f_{\alpha}\right)_{\alpha \in Q_{1}}\right)$ de um $k Q$-módulo injetivo indecomponível associado ao vértice $a \in\{1,2, \ldots, n\}$. Para cada $i$, a $\operatorname{dim}_{k} V_{i}$ é igual ao número de caminhos em $Q$ de $i$ para $a$. Para mais detalhes, ver por exemplo, [ASS06] (III.2). Para uma álgebra $\Lambda$ denotaremos por $I_{\Lambda}(i)$ o injetivo indecomponível associado ao vértice $i \in Q_{0}$.

Observe que dada uma aljava $Q$ com poço no vértice 1 e uma subaljava plena $Q^{\prime}$ de $Q$ obtida excluíndo o poço 1 , ou seja, $Q_{0}^{\prime}=Q_{0} \backslash\{1\}$, então para cada $j$ em $Q_{0}^{\prime}$, o injetivo indecomponível $I_{k Q^{\prime}}(j)$ de $k Q^{\prime}$ pode ser visto como o injetivo indecomponível $I_{k Q}(j)$ de $k Q$, pois na representação desse último temos que $V_{1}=0$, uma vez que não existem caminhos de 1 para $j$ e os outros caminhos são os mesmos pela plenitude de $Q^{\prime}$. 


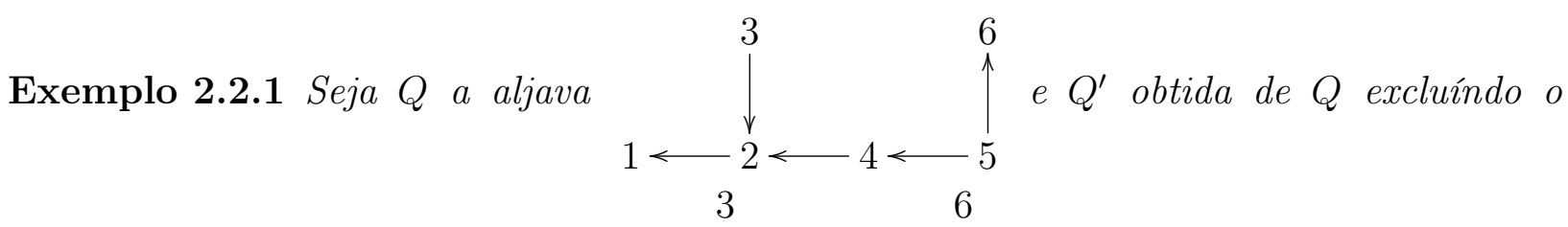

vértice 1, ou seja, $Q^{\prime}$ é a aljava
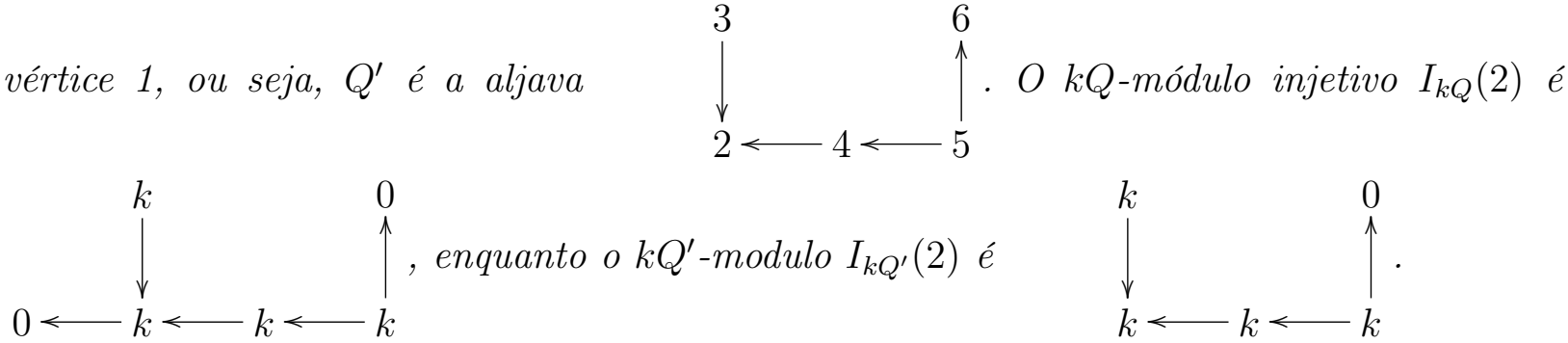

Observemos que se $M=\left(\left(V_{i}\right)_{i \in Q_{0}},\left(f_{\alpha}\right)_{\alpha \in Q_{1}}\right)$ é uma $Q$-representação com $f_{\alpha}$ sobrejetora para todo $\alpha$ em $Q_{1}$ então cada somando $\left(\left(W_{i}\right)_{i \in Q_{0}},\left(g_{\alpha}\right)_{\alpha \in Q_{1}}\right)$ de $M$ também tem a mesma propriedade, ou seja, cada $g_{\alpha}$ é sobrejetora.

De fato, basta olhar, para cada $\alpha: i \rightarrow j$ em $Q_{1}$, o diagrama comutativo

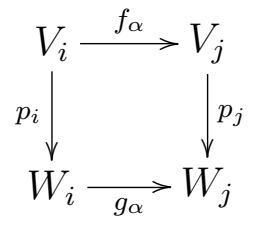

onde $p_{i}$ e $p_{j}$ são as projeções. Como $f_{\alpha}$ é sobrejetora então $p_{j} f_{\alpha}=g_{\alpha} p_{i}$ também é sobrejetora e portanto $g_{\alpha}$ também é.

Segue dessa observação que basta olharmos o que acontece com os módulos indecomponíveis.

Finalmente, iremos demonstrar o seguinte teorema.

Teorema 2.2.2 Seja $Q$ uma aljava de tipo Dynkin com um único poço.

Se $M=\left(\left(V_{i}\right)_{i \in Q_{0}},\left(f_{\alpha}\right)_{\alpha \in Q_{1}}\right)$ é uma $Q$-representação com $f_{\alpha}$ sobrejetora para todo $\alpha$ em $Q_{1}$, então $M$ é a representação de um módulo injetivo.

Prova. Seja $Q$ uma aljava de tipo Dynkin com um único poço e suponha $Q_{0}=\{1,2, \ldots, n\}$. Se $M=\left(\left(V_{i}\right)_{i \in Q_{0}},\left(f_{\alpha}\right)_{\alpha \in Q_{1}}\right)$ é uma $Q$-representação de um $k Q$-módulo indecomponível então o vetor dimensão de $M, \underline{\operatorname{dim}} M=\left(x_{1}, x_{2}, \ldots, x_{n}\right)$, é uma raiz da forma quadrática $q_{Q}$ associada à $Q$. Suponhamos que para cada $\alpha$ em $Q_{1}$ a aplicação $f_{\alpha}$ é sobrejetora. Nesse caso temos que $x_{t(\alpha)} \leq x_{s(\alpha)}$ para cada $\alpha$ em $Q_{1}$. Vejamos que, nesse caso, o vetor $\left(x_{1}, x_{2}, \ldots, x_{n}\right)$ é o vetor dimensão de um módulo injetivo. Faremos a prova caso a caso.

Caso $\mathbb{A}_{n}$

Fixemos a seguinte numeração para os vértices do grafo associado à $Q$ :

$$
1-2-\cdots-n-1-n
$$

A forma quadrática de $Q$ é $q_{Q}\left(y_{1}, y_{2}, \ldots, y_{n}\right)=\sum_{i=1}^{n} y_{i}^{2}-\sum_{i=1}^{n-1} y_{i} y_{i+1}$. Como $\left(x_{1}, x_{2}, \ldots, x_{n}\right)$ é uma raiz de $q_{Q}$ então $\sum_{i=1}^{n} x_{i}^{2}-\sum_{i=1}^{n-1} x_{i} x_{i+1}=1$ e multiplicando por 2 e reorganizando 
os termos como uma soma de quadrados temos que $x_{1}^{2}+x_{n}^{2}+\sum_{i=1}^{n-1}\left(x_{i}-x_{i+1}\right)^{2}=2$. Segue que $x_{1}$ e $x_{n}$ são no máximo 1 .

Seja $i$ o único poço de $Q$ então como cada $f_{\alpha}$ é sobrejetora temos as seguintes desigualdades: $x_{i} \leq x_{i+1} \leq \ldots \leq x_{n}$ e $x_{i} \leq x_{i-1} \leq \ldots \leq x_{1}$. Portanto, cada $x_{j}$ é também no máximo 1 . Se $x_{i}=1$ então $x_{j}=1$ para todo $j \in\{1,2, \ldots, n\}$ e nesse caso $M$ é o injetivo $I(i)$ associado ao vértice $i$. Caso contrário como $M$ é indecomponível temos que $x_{j}=0$ para todo $j>i$ ou $x_{j}=0$ para todo $j<i$. Seja $t>i$ tal que $x_{t}=1$ e $x_{t-1}=0$. Neste caso, $x_{j}=1$ para todo $j \geq t$ e $x_{j}=0$ para todo $j<t$ e, portanto, $M=I(t)$. Analogamente para $t<i$.

Caso $\mathbb{D}_{n}$

Fixemos a seguinte numeração para os vértices do grafo associado à $Q$ :

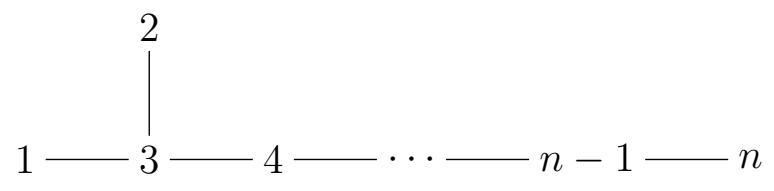

Nesse caso, multiplicando por 4 a expressão $q_{Q}\left(x_{1}, \ldots, x_{n}\right)=1$ e reorganizando os termos de $4 q_{Q}\left(x_{1}, \ldots, x_{n}\right)=4$ temos que $\left(2 x_{1}-x_{3}\right)^{2}+\left(2 x_{2}-x_{3}\right)^{2}+2 \sum_{i=3}^{n-1}\left(x_{i}-x_{i+1}\right)^{2}+2 x_{n}^{2}=4$. Nesse caso, $x_{n}$ é no máximo 1.

\section{Poço em 1}

Temos as seguintes desigualdades: $x_{1} \leq x_{3} \leq x_{4} \leq \ldots \leq x_{n} \leq 1$ e $x_{1} \leq x_{3} \leq x_{2}$.

Se $x_{1}$ é não nulo então $x_{i}=1$ para $i \in\{1,3,4, \ldots, n\}$. Substituindo esses termos em $4 q_{Q}\left(x_{1}, \ldots, x_{n}\right)=4$ concluímos que $x_{2}=1 \mathrm{e}$, portanto, $M=I(1)$.

Suponha agora que $x_{1}=0$. Nesse caso seja $Q^{\prime}$ a subaljava plena de $Q$ obtida excluíndo o vértice 1. Então, $Q^{\prime}$ é de tipo $\mathbb{A}_{n-1}$ com um único poço em 3 . Podemos olhar $M$ como um $k Q^{\prime}$-módulo e pela parte anterior segue que $M$ é um $k Q^{\prime}$-módulo injetivo. Basta observar que para $i \geq 2$ temos que $I_{k Q^{\prime}}(i) \cong I_{k Q}(i)$ concluíndo o resultado para esse caso.

\section{Poço em 2}

Simétrico ao caso anterior.

\section{Poço em 3}

Temos as seguintes desigualdades: $x_{3} \leq x_{4} \leq \ldots \leq x_{n} \leq 1, x_{3} \leq x_{1}$ e $x_{3} \leq x_{2}$.

Suponhamos inicialmente que $x_{3}$ é não nulo. Então $x_{i}=1$ para $i \geq 3$. Falta encontrar $x_{2}$ e $x_{1}$. Temos nesse caso, $\left(2 x_{1}-x_{3}\right)^{2}+\left(2 x_{2}-x_{3}\right)^{2}+2=4$. Como $x_{1}, x_{2} \geq 1$ segue que $x_{1}=x_{2}=1$ e, portanto, $M=I(3)$.

Se $x_{3}=0$, como $M$ é indecomponível então $M=S(1)=I(1)$ ou $M=S(2)=I(2)$ ou $M$ é um $k Q^{\prime}$-módulo onde $Q^{\prime}$ é a subaljava plena de $Q$ tal que $Q_{0}^{\prime}=\{4,5, \ldots, n\}$. Nesse caso $Q^{\prime}$ é de tipo $\mathbb{A}_{n-3}$ com um único vértice em 4 e para os vértices de $Q^{\prime}$ os injetivos de $k Q$ e $k Q^{\prime}$ coincidem. Segue que $M$ é injetivo. 
Poço em $4 \leq t \leq n$

Temos as seguintes desigualdades: $x_{t} \leq x_{t+1} \leq \ldots \leq x_{n} \leq 1, x_{t} \leq x_{t-1} \leq \ldots \leq x_{4} \leq x_{3} \leq$ $x_{2}$ e $x_{3} \leq x_{1}$.

Se $x_{t}$ é não nulo então $x_{i}=1$ para $i \geq t$ e $x_{i} \geq 1$ para $i<t$. Em $4 q_{Q}\left(x_{1}, \ldots, x_{n}\right)=4$ temos $(*):\left(2 x_{1}-x_{3}\right)^{2}+\left(2 x_{2}-x_{3}\right)^{2}+2 \sum_{i=3}^{t-1}\left(x_{i}-x_{i+1}\right)^{2}=2$. Então $\left(x_{t-1}-1\right)^{2} \leq 2 \mathrm{e}$, portanto, $1 \leq x_{t-1} \leq 2$. Vejamos que $x_{t-1} \neq 2$. Se não fosse esse o caso, substituindo em $(*)$ teríamos $x_{t-1}=x_{t-2}=\ldots=x_{4}=x_{3}=2$ e $x_{2}=1$, uma contradição pois $x_{3} \leq x_{2}$. Logo, $x_{t-1}=1$. Repetindo o argumento temos que $x_{i}=1$ para todo $i$ e nesse caso $M=I(t)$.

Se $x_{t}=0$ então como $M$ é indecomponível temos que $M$ é um $k Q^{\prime}$-módulo onde $Q^{\prime}$ é a subaljava plena de $Q$ com $Q_{0}^{\prime}=\{t+1, t+2, \ldots, n\}$ ou $M$ é um $k Q^{\prime \prime}$-módulo onde $Q^{\prime \prime}$ é a subaljava plena de $Q$ com $Q_{0}^{\prime \prime}=\{1,2, \ldots, t-1\}$. Então, $Q^{\prime}$ é de tipo $\mathbb{A}_{n-t}$ com único poço em $t+1$ e nesse caso a conclusão segue de um caso anterior, pois os injetivos de $k Q^{\prime}$ são $k Q$-injetivos.

Se $M$ é um $k Q^{\prime \prime}$-módulo, suponha que $x_{t-1}=1$. Em $(*)$ concluímos que $1 \leq x_{t-2} \leq 2$ Se $x_{t-2}=2$ segue que $x_{t-2}=\ldots=x_{4}=x_{3}=2$ e $x_{2}=1$, uma contradição pois $x_{3} \leq x_{2}$. Logo, $x_{t-2}=1$. Repetindo o argumento chegamos a $M=I(t-1)$. Analogamente, se $x_{l}=1$ e $x_{l-1}=0$ então $M=I(l)$ concluíndo esse caso.

Caso $\mathbb{E}_{6}$

Fixemos a seguinte numeração para os vértices do grafo associado à $Q$ :

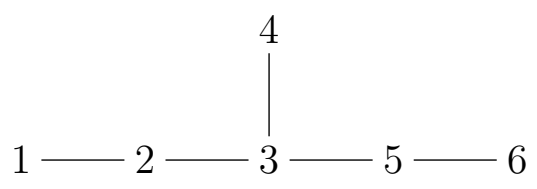

Fazendo $36 q_{Q}\left(x_{1}, \ldots, x_{6}\right)=36$ temos $\left(6 x_{1}-3 x_{2}\right)^{2}+\left(6 x_{6}-3 x_{5}\right)^{2}+3\left(3 x_{2}-2 x_{3}\right)^{2}+$ $3\left(3 x_{4}-2 x_{3}\right)^{2}+3\left(3 x_{5}-2 x_{3}\right)^{2}+9 x_{4}^{2}=36$. Nesse caso temos que $x_{4} \leq 2$. Se $x_{4}=2$ então $\underline{\operatorname{dim}} M=(1,2,3,2,2,1)$ uma contradição com a propriedade de $M$ que cada $f_{\alpha}$ é sobrejetora e que $Q$ tem um único poço. Portanto, temos que $x_{4} \leq 1$.

\section{Poço em 1}

Temos as seguintes desigualdades: $x_{3} \leq x_{5} \leq x_{6}$ e $x_{1} \leq x_{2} \leq x_{3} \leq x_{4} \leq 1$.

Se $x_{1}=1$ então $x_{1}=x_{2}=x_{3}=x_{4}=1$. Substituindo em $36 q_{Q}\left(x_{1}, \ldots, x_{6}\right)=36$ temos que $x_{5}=x_{6}=1$ e, portanto, $M=I(1)$.

Se $x_{1}=0$ então $M$ é um $k Q^{\prime}$-módulo onde $Q^{\prime}$ é a subaljava plena de $Q$ cujo conjunto de vértices é $\{2,3,4,5,6\}$ que é uma aljava de tipo $\mathbb{D}_{5}$ com um único poço em 2 . Logo, $M$ é um $k Q^{\prime}$-módulo injetivo e, portanto, é também um $k Q$-módulo injetivo.

\section{Poço em 2}

Temos as seguintes desigualdades: $x_{2} \leq x_{1}, x_{2} \leq x_{3} \leq x_{4} \leq 1$ e $x_{3} \leq x_{5} \leq x_{6}$.

Se $x_{2}=1$ então $x_{2}=x_{3}=x_{4}=1$. Substituíndo em $36 q_{Q}\left(x_{1}, \ldots, x_{6}\right)=36$ temos que 
$x_{1}=x_{5}=x_{6}=1$ e, portanto, $M=I(2)$.

Se $x_{2}=0$ então, como $M$ é indecomponível, $M$ é o simples $S(1)$ que é injetivo ou $M$ é um $k Q^{\prime}$-módulo onde $Q^{\prime}$ é a subaljava plena de $Q$ cujo conjunto de vértices é $\{3,4,5,6\}$ que é uma aljava de tipo $\mathbb{A}_{4}$ com um único poço em 3. Logo, $M$ é um $k Q^{\prime}$-módulo injetivo e, portanto, é também um $k Q$-módulo injetivo.

\section{Poço em 3}

Temos as seguintes desigualdades: $x_{3} \leq x_{4} \leq 1, x_{3} \leq x_{2} \leq x_{1}$ e $x_{3} \leq x_{5} \leq x_{6}$.

Se $x_{3}=1$ então $x_{4}=1$. Substituíndo em $36 q_{Q}\left(x_{1}, \ldots, x_{6}\right)=36$ concluímos que $x_{1}=x_{6}=$ 1 e, portanto, $M=I(3)$.

Se $x_{3}=0$, como $M$ é indecomponível, então $M=S(4)=I(4)$ ou $M$ é um $k Q^{\prime}$-módulo ou $M$ é um $k Q^{\prime \prime}$-módulo onde $Q^{\prime}$ e $Q^{\prime \prime}$ são subaljavas plenas de $Q$ cujos conjuntos de vértices são, respectivamente, $Q_{0}^{\prime}=\{1,2\}$ e $Q_{0}^{\prime \prime}=\{5,6\}$. Então, $Q^{\prime}$ e $Q^{\prime \prime}$ são aljavas de tipo $\mathbb{A}_{2}$ com um único poço, respectivamente, em 2 e 5 . Segue que $M$ é um $k Q^{\prime}$-módulo injetivo ou um $k Q^{\prime \prime}$-módulo injetivo. Em qualquer caso, $M$ é injetivo em $k Q$.

\section{Poço em 4}

Temos as seguintes desigualdades: $x_{4} \leq x_{3} \leq x_{5} \leq x_{6}$ e $x_{3} \leq x_{2} \leq x_{1}$.

Se $x_{4}=1$, então $\left(6 x_{1}-3 x_{2}\right)^{2}+\left(6 x_{6}-3 x_{5}\right)^{2}+3\left(3 x_{2}-2 x_{3}\right)^{2}+3\left(3-2 x_{3}\right)^{2}+3\left(3 x_{5}-2 x_{3}\right)^{2}=27$ e, portanto, $\left(3-2 x_{3}\right)^{2} \leq 9$, ou seja, $x_{3} \leq 3$. Denote por $(*)$ a igualdade $\left(6 x_{1}-3 x_{2}\right)^{2}+\left(6 x_{6}-\right.$ $\left.3 x_{5}\right)^{2}+3\left(3 x_{2}-2 x_{3}\right)^{2}+3\left(3 x_{5}-2 x_{3}\right)^{2}=27-3\left(3-2 x_{3}\right)^{2}$

Se $x_{3}=3$ então em $(*)$ temos $\left(6 x_{1}-3 x_{2}\right)^{2}+\left(6 x_{6}-3 x_{5}\right)^{2}+3\left(3 x_{2}-2 x_{3}\right)^{2}+3\left(3 x_{5}-2 x_{3}\right)^{2}=0$ e, portanto, $x_{5}=2$ uma contradição. Se $x_{3}=2$ então $\left(6 x_{1}-3 x_{2}\right)^{2}+\left(6 x_{6}-3 x_{5}\right)^{2}+3\left(3 x_{2}-\right.$ $\left.2 x_{3}\right)^{2}+3\left(3 x_{5}-2 x_{3}\right)^{2}=24$. Para $i=2,5$ segue que $3\left(3 x_{i}-4\right)^{2} \leq 24$ e, portanto, como $x_{i} \geq x_{3}=2$, segue que $x_{i}=2$. Mas, daí teríamos $x_{1}=1$ uma contradição. Logo, $x_{3}=1$. Em $(*)$ temos, para $i=2,5$ temos $3\left(3 x_{i}-2\right)^{2} \leq 24$ e, portanto, $x_{2}=x_{5}=1$. Substituíndo em $(*)$ concluímos que $x_{1}=x_{6}=1$ e, portanto, $M=I(4)$.

Se $x_{4}=0$ então $M$ é um $k Q^{\prime}$-módulo onde $Q^{\prime}$ é a subaljava de $Q$ cujo conjunto de vértices é $Q_{0}^{\prime}=\{1,2,3,5,6\}$ que é uma aljava de tipo $\mathbb{A}_{5}$ com único poço em 3 . Logo, $M$ é injetivo em $k Q^{\prime}$ e, portanto, é injetivo em $k Q$.

\section{Poço em 5 ou em 6}

Esses casos são simétricos aos casos em que o poço está em 2 ou em 1.

\section{Caso $\mathbb{E}_{7}$}

Fixemos a seguinte numeração para os vértices do grafo associado à $Q$ :

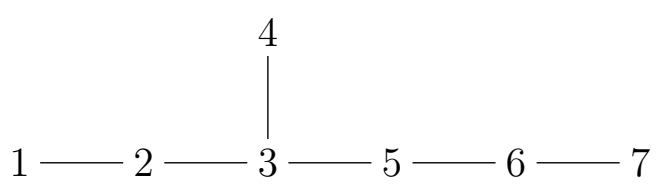

Fazendo $24 q_{Q}\left(x_{1}, \ldots, x_{n}\right)=24$ temos $6 x_{1}^{2}+6\left(2 x_{7}-x_{6}\right)^{2}+2\left(3 x_{1}-2 x_{2}\right)^{2}+2\left(3 x_{6}-2 x_{5}\right)^{2}+$ 
$\left(4 x_{2}-3 x_{3}\right)^{2}+\left(4 x_{5}-3 x_{3}\right)^{2}+6\left(2 x_{4}-x_{3}\right)^{2}=24$. Segue que $x_{1} \leq 2$. Se $x_{1}=2$, então chegamos a $\underline{\operatorname{dim}} M=(2,3,4,2,3,2,1)$, o que é uma contradição com a propriedade de $M$ em que cada $f_{\alpha}$ é sobrejetora e que $Q$ tem um único poço. Portanto, $x_{1} \leq 1$.

\section{Poço em 1}

Temos as seguintes desigualdades: $x_{1} \leq x_{2} \leq x_{3} \leq x_{5} \leq x_{6} \leq x_{7}$ e $x_{3} \leq x_{4}$.

Se $x_{1}=1$, então temos $6\left(2 x_{7}-x_{6}\right)^{2}+2\left(3-2 x_{2}\right)^{2}+2\left(3 x_{6}-2 x_{5}\right)^{2}+\left(4 x_{2}-3 x_{3}\right)^{2}+\left(4 x_{5}-\right.$ $\left.3 x_{3}\right)^{2}+6\left(2 x_{4}-x_{3}\right)^{2}=18$. Segue que $2\left(3-2 x_{2}\right)^{2} \leq 18$ e, portanto, $x_{2} \leq 3$.

- Se $x_{2}=3$ então $x_{3}=4$ e $x_{5}=3$, o que contradiz $x_{3} \leq x_{5}$.

- Se $x_{2}=2$ então $6\left(2 x_{7}-x_{6}\right)^{2}+2\left(3 x_{6}-2 x_{5}\right)^{2}+\left(8-3 x_{3}\right)^{2}+\left(4 x_{5}-3 x_{3}\right)^{2}+6\left(2 x_{4}-x_{3}\right)^{2}=16$. Então $\left(8-3 x_{3}\right)^{2} \leq 16$ e, portanto, $2 \leq x_{3} \leq 4$. Vejamos que nenhum desses casos pode acotecer.

- Se $x_{3}=4$ então $x_{4}=2$, que é uma contradição pois $x_{3} \leq x_{4}$.

- Se $x_{3}=3$ então $x_{4} \leq 2$, que é uma contradição pois $x_{3} \leq x_{4}$

- Se $x_{3}=2$ então $x_{4}=1$, o que é novamente uma contradição.

Portanto, temos que $x_{2}=1$. Substituindo em $24 q_{Q}\left(x_{1}, \ldots, x_{n}\right)=24$ chegamos em $6\left(2 x_{7}-\right.$ $\left.x_{6}\right)^{2}+2\left(3 x_{6}-2 x_{5}\right)^{2}+\left(4-3 x_{3}\right)^{2}+\left(4 x_{5}-3 x_{3}\right)^{2}+6\left(2 x_{4}-x_{3}\right)^{2}=16$ e daí $\left(4-3 x_{3}\right)^{2} \leq 16$, o que implica em $x_{3} \leq 2$. Vejamos que $x_{3} \neq 2$.

- Se $x_{3}=2$, então teríamos $6\left(2 x_{7}-x_{6}\right)^{2}+2\left(3 x_{6}-2 x_{5}\right)^{2}+\left(4 x_{5}-6\right)^{2}+6\left(2 x_{4}-2\right)^{2}=12$ e de $6\left(2 x_{4}-2\right)^{2} \leq 12$ segue que $x_{4} \leq 1$, uma contradição pois $x_{3} \leq x_{4}$.

Portanto, $x_{3}=1$. Agora temos $6\left(2 x_{7}-x_{6}\right)^{2}+2\left(3 x_{6}-2 x_{5}\right)^{2}+\left(4 x_{5}-3\right)^{2}+6\left(2 x_{4}\right)^{2}=15 \mathrm{e}$ disto segue que $x_{4}=x_{5}=x_{7}=1 \mathrm{e}$, portanto, $M=I(1)$.

Se $x_{1}=0$ então $M$ é um $k Q^{\prime}$-módulo onde $Q^{\prime}$ é a subaljava plena de $Q$ cujo conjunto de vértices é $Q_{0}^{\prime}=\{2,3,4,5,6,7\}$, que é uma aljava de tipo $\mathbb{D}_{6}$ com único poço em 2 . Segue então que $M$ é um $k Q^{\prime}$-módulo injetivo e, portanto, um $k Q$-módulo injetivo.

\section{Poço em 2}

Temos as seguintes desigualdades: $x_{2} \leq x_{1} \leq 1, x_{2} \leq x_{3} \leq x_{4}$ e $x_{3} \leq x_{5} \leq x_{6} \leq x_{7}$.

Se $x_{2}=1$ então $x_{1}=1$. Em $24 q_{Q}\left(x_{1}, \ldots, x_{n}\right)=24$ temos $6\left(2 x_{7}-x_{6}\right)^{2}+2\left(3 x_{6}-2 x_{5}\right)^{2}+$ $\left(4-3 x_{3}\right)^{2}+\left(4 x_{5}-3 x_{3}\right)^{2}+6\left(2 x_{4}-x_{3}\right)^{2}=16$. De $\left(4-3 x_{3}\right)^{2} \leq 16$ temos que $x_{3} \leq 2$. Se $x_{3}=2$ então $x_{4} \leq 1$, o que é uma contradição. Logo, $x_{3}=1$. Então $6\left(2 x_{7}-x_{6}\right)^{2}+2\left(3 x_{6}-\right.$ $\left.2 x_{5}\right)^{2}+\left(4 x_{5}-3\right)^{2}+6\left(2 x_{4}-1\right)^{2}=15$ e daí segue que $x_{4}=x_{5}=x_{6}=x_{7}=1$ e, portanto, $M=I(2)$.

Se $x_{2}=0$ então, como $M$ é indecomponível, $M=S(1)=I(1)$ ou $M$ é um $k Q^{\prime}$-módulo onde $Q^{\prime}$ é a subaljava plena de $Q$ cujo conjunto de vértices é $Q_{0}^{\prime}=\{3,4,5,6,7\}$, que é uma aljava de tipo $\mathbb{A}_{5}$ com único poço em 3. Segue então que $M$ é um $k Q^{\prime}$-módulo injetivo e, portanto, um $k Q$-módulo injetivo. 


\section{Poço em 3}

Temos as seguintes desigualdades: $x_{3} \leq x_{4}, x_{3} \leq x_{2} \leq x_{1} \leq 1$ e $x_{3} \leq x_{5} \leq x_{6} \leq x_{7}$.

Se $x_{3}=1$ então $x_{2}=x_{1}=1$. Substituindo em $24 q_{Q}\left(x_{1}, \ldots, x_{n}\right)=24$ temos que $6\left(2 x_{7}-\right.$ $\left.x_{6}\right)^{2}+2\left(3 x_{6}-2 x_{5}\right)^{2}+\left(4 x_{5}-3\right)^{2}+6\left(2 x_{4}-1\right)^{2}=15$ e daí segue que $x_{4}=x_{5}=x_{6}=x_{7}=1$ e, portanto, $M=I(3)$.

Se $x_{3}=0$, como $M$ é indecomponível, então $M=S(4)=I(4)$ ou $M$ é um $k Q^{\prime}$-módulo ou $M$ é um $k Q^{\prime \prime}$-módulo, onde $Q^{\prime}$ e $Q^{\prime \prime}$ são subaljavas plenas de $Q$ cujos conjuntos de vértices são $Q_{0}^{\prime}=\{1,2\}$ e $Q_{0}^{\prime \prime}=\{5,6,7\}$. Então $Q^{\prime}$ e $Q^{\prime \prime}$ são aljavas de tipo $\mathbb{A}_{2}$ com único poço em 2 e $\mathbb{A}_{3}$ com único poço em 5 , respectivamente. Segue que $M$ é injetivo em $k Q^{\prime}$ ou injetivo em $k Q^{\prime \prime}$. Em qualquer caso, $M$ é injetivo em $k Q$.

\section{Poço em 4}

Temos as seguintes desigualdades: $x_{4} \leq x_{3} \leq x_{2} \leq x_{1} \leq 1$ e $x_{3} \leq x_{5} \leq x_{6} \leq x_{7}$.

Se $x_{4}=1$ então $x_{4}=x_{3}=x_{2}=x_{1}=1$ e de $24 q_{Q}\left(x_{1}, \ldots, x_{n}\right)=24$ concluímos também que $x_{5}=x_{6}=x_{7}=1 \mathrm{e}$, portanto, $M=I(4)$.

Se $x_{4}=0$ então $M$ é um $k Q^{\prime}$-módulo onde $Q^{\prime}$ é a subaljava plena de $Q$ cujo conjunto de vértices é $Q_{0}^{\prime}=\{1,2,3,5,6,7\}$, que é uma aljava de tipo $\mathbb{A}_{6}$ com único poço em 3 . Segue então que $M$ é um $k Q^{\prime}$-módulo injetivo e, portanto, um $k Q$-módulo injetivo.

\section{Poço em 5}

Temos as seguintes desigualdades: $x_{5} \leq x_{6} \leq x_{7}, x_{5} \leq x_{3} \leq x_{2} \leq x_{1} \leq 1$ e $x_{3} \leq x_{4}$.

Se $x_{5}=1$ então $x_{5}=x_{3}=x_{2}=x_{1}=1$ e de $24 q_{Q}\left(x_{1}, \ldots, x_{n}\right)=24$ segue que $x_{4}=x_{6}=$ $x_{7}=1$ e, portanto, $M=I(5)$.

Se $x_{5}=0$ então $M$ é um $k Q^{\prime}$-módulo ou $M$ é um $k Q^{\prime \prime}$-módulo, onde $Q^{\prime}$ e $Q^{\prime \prime}$ são subaljavas plenas de $Q$ cujos conjuntos de vértices são $Q_{0}^{\prime}=\{6,7\}$ e $Q_{0}^{\prime \prime}=\{1,2,3,4\}$. Então $Q^{\prime}$ e $Q^{\prime \prime}$ são aljavas de tipo $\mathbb{A}_{2}$ com único poço em 6 e $\mathbb{A}_{4}$ com único poço em 3 , respectivamente. Segue que $M$ é injetivo em $k Q^{\prime}$ ou injetivo em $k Q^{\prime \prime}$. Em qualquer caso, $M$ é injetivo em $k Q$.

\section{Poço em 6}

Temos as seguintes desigualdades: $x_{6} \leq x_{7}, x_{6} \leq x_{5} \leq x_{3} \leq x_{2} \leq x_{1} \leq 1$ e $x_{3} \leq x_{4}$.

Se $x_{6}=1$ então $x_{6}=x_{5}=x_{3}=x_{2}=x_{1}=1 \mathrm{e}$, portanto, $x_{4}=x_{6}=x_{7}=1$. Nesse caso $M=I(6)$.

Se $x_{6}=0$ então $M=S(7)=I(7)$ ou $M$ é um $k Q^{\prime}$-módulo onde $Q^{\prime}$ é a subaljava plena de $Q$ cujo conjunto de vértices é $Q_{0}^{\prime}=\{1,2,3,4,5\}$, que é uma aljava de tipo $\mathbb{D}_{5}$ com único poço em 5. Segue então que $M$ é um $k Q^{\prime}$-módulo injetivo e, portanto, um $k Q$-módulo injetivo.

\section{Poço em 7}

Temos as seguintes desigualdades: $x_{7} \leq x_{6} \leq x_{5} \leq x_{3} \leq x_{2} \leq x_{1} \leq 1$ e $x_{3} \leq x_{4}$.

Se $x_{7}=1$ então $x_{7}=x_{6}=x_{5}=x_{3}=x_{2}=x_{1}=1$. Segue que $x_{4}=1$ e, portanto, $M=I(7)$. 
Se $x_{7}=0$ então $M$ é um $k Q^{\prime}$-módulo onde $Q^{\prime}$ é a subaljava plena de $Q$ cujo conjunto de vértices é $Q_{0}^{\prime}=\{1,2,3,4,5,6\}$ que é uma aljava de tipo $\mathbb{E}_{6}$ com único poço em 6 . Segue então que $M$ é um $k Q^{\prime}$-módulo injetivo e, portanto, um $k Q$-módulo injetivo.

Caso $\mathbb{E}_{8}$

Fixemos a seguinte numeração para os vértices do grafo associado à $Q$ :

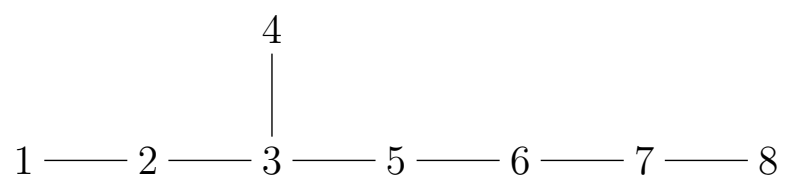

Fazendo $120 q_{Q}\left(x_{1}, \ldots, x_{n}\right)=120$ temos $30 x_{8}^{2}+10\left(3 x_{8}-2 x_{7}\right)^{2}+5\left(4 x_{7}-3 x_{6}\right)^{2}+3\left(5 x_{6}-\right.$ $\left.4 x_{5}\right)^{2}+30\left(2 x_{1}-x_{2}\right)^{2}+2\left(6 x_{5}-5 x_{3}\right)^{2}+10\left(3 x_{2}-2 x_{3}\right)^{2}+30\left(2 x_{4}-x_{3}\right)^{2}=120$. Então, $x_{8} \leq 2$. Suponha $x_{8}=2$. Então $\underline{\operatorname{dim}} M=(2,4,6,3,5,4,3,2)$, o que é uma contradição com a propriedade de $M$ em que cada $f_{\alpha}$ é sobrejetora e que $Q$ tem um único poço. Portanto $x_{8} \leq 1$.

\section{Poço em 1}

Temos as seguintes desigualdades: $x_{1} \leq x_{2} \leq x_{3} \leq x_{5} \leq x_{6} \leq x_{7} \leq x_{8} \leq 1$ e $x_{3} \leq x_{4}$.

Se $x_{1}=1$ então $x_{i}=1$ para todo $i=1, \ldots, 8$ e nesse caso $M=I(1)$.

Se $x_{1}=0$ então $M$ é um $k Q^{\prime}$-módulo, onde $Q^{\prime}$ é a subaljava plena de $Q$ cujo conjunto de vértices é $Q_{0}^{\prime}=\{2,3,4,5,6,7,8\}$, que é uma aljava de tipo $\mathbb{D}_{7}$ com único poço em 2 . Segue então que $M$ é um $k Q^{\prime}$-módulo injetivo e, portanto, um $k Q$-módulo injetivo.

\section{Poço em 2}

Temos as seguintes desigualdades: $x_{2} \leq x_{1}, x_{2} \leq x_{3} \leq x_{5} \leq x_{6} \leq x_{7} \leq x_{8} \leq 1$ e $x_{3} \leq x_{4}$.

Se $x_{2}=1$ então $x_{2}=x_{3}=x_{5}=x_{6}=x_{7}=x_{8}=1$. Substituindo em $120 q_{Q}\left(x_{1}, \ldots, x_{n}\right)=$ 120 chegamos em $x_{4}=1$ e, portanto, $M=I(2)$.

Se $x_{2}=0$ então, como $M$ é indecomponível, temos que $M=S(1)=I(1)$ ou $M$ é um $k Q^{\prime}$ módulo onde $Q^{\prime}$ é a subaljava plena de $Q$ cujo conjunto de vértices é $Q_{0}^{\prime}=\{3,4,5,6,7,8\}$, que é uma aljava de tipo $\mathbb{A}_{6}$ com único poço em 3 . Segue então que $M$ é um $k Q^{\prime}$-módulo injetivo e, portanto, um $k Q$-módulo injetivo.

\section{Poço em 3}

Temos as seguintes desigualdades: $x_{3} \leq x_{2} \leq x_{1}, x_{3} \leq x_{5} \leq x_{6} \leq x_{7} \leq x_{8} \leq 1$ e $x_{3} \leq x_{4}$.

Se $x_{3}=1$ então $x_{3}=x_{5}=x_{6}=x_{7}=x_{8}=1$. Daí é fácil verfificar que $x_{4}=x_{2}=x_{1}=1$ e, portanto, $M=I(3)$.

Se $x_{3}=0$ então, como $M$ é indecomponível, temos que $M=S(4)=I(4)$ ou $M$ é um $k Q^{\prime}$-módulo ou um $k Q^{\prime \prime}$-módulo onde $Q^{\prime}$ e $Q^{\prime \prime}$ são subaljavas plenas de $Q$ cujos conjuntos de vértices são $Q_{0}^{\prime}=\{1,2\}$ e $Q_{0}^{\prime \prime}=\{5,6,7,8\}$. Então $Q^{\prime}$ e $Q^{\prime \prime}$ são aljavas de tipo $\mathbb{A}_{2}$ com único poço em 2 e $\mathbb{A}_{4}$ com único poço em 5 , respectivamente. Segue que $M$ é injetivo em $k Q^{\prime}$ ou injetivo em $k Q^{\prime \prime}$. Em qualquer caso, $M$ é injetivo em $k Q$. 


\section{Poço em 4}

Temos as seguintes desigualdades: $x_{4} \leq x_{3} \leq x_{5} \leq x_{6} \leq x_{7} \leq x_{8} \leq 1$ e $x_{3} \leq x_{2} \leq x_{1}$.

Se $x_{4}=1$ então $x_{4}=x_{3}=x_{5}=x_{6}=x_{7}=x_{8}=1$ e segue que $x_{1}=x_{2}=1$. Portanto, $M=I(4)$.

Se $x_{4}=0$ então $M$ é um $k Q^{\prime}$-módulo onde $Q^{\prime}$ é a subaljava plena de $Q$ cujo conjunto de vértices é $Q_{0}^{\prime}=\{1,2,3,5,6,7,8\}$ que é uma aljava de tipo $\mathbb{A}_{7}$ com único poço em 3 . Segue então que $M$ é um $k Q^{\prime}$-módulo injetivo e, portanto, um $k Q$-módulo injetivo.

\section{Poço em 5}

Temos as seguintes desigualdades: $x_{5} \leq x_{6} \leq x_{7} \leq x_{8} \leq 1$ e $x_{5} \leq x_{3} \leq x_{2} \leq x_{1}$ e $x_{3} \leq x_{4}$.

Se $x_{5}=1$ então $x_{5}=x_{6}=x_{7}=x_{8}=1$. Substituindo em $120 q_{Q}\left(x_{1}, \ldots, x_{n}\right)=120$ temos $30\left(2 x_{1}-x_{2}\right)^{2}+2\left(6-5 x_{3}\right)^{2}+10\left(3 x_{2}-2 x_{3}\right)^{2}+30\left(2 x_{4}-x_{3}\right)^{2}=72$. Então $2\left(6-5 x_{3}\right)^{2} \leq 72$ e, portanto, $x_{3} \leq 2$. Se $x_{3}=2$ então $30\left(2 x_{1}-x_{2}\right)^{2}+10\left(3 x_{2}-4\right)^{2}+30\left(2 x_{4}-2\right)^{2}=40$ e então $x_{2}=2$ e $x_{1}=1$, que é uma contradição, pois $x_{2} \leq x_{1}$. Logo $x_{3}=1$. Daí $30\left(2 x_{1}-x_{2}\right)^{2}+$ $10\left(3 x_{2}-2\right)^{2}+30\left(2 x_{4}-1\right)^{2}=70$ e disto segue que $x_{4}=x_{2}=x_{1}=1$ e, portanto, $M=I(5)$.

Se $x_{5}=0$ então, como $M$ é indecomponível, temos que $M$ é um $k Q^{\prime}$-módulo ou um $k Q^{\prime \prime}$-módulo onde $Q^{\prime}$ e $Q^{\prime \prime}$ são subaljavas plenas de $Q$ cujos conjuntos de vértices são $Q_{0}^{\prime}=$ $\{1,2,3,4\}$ e $Q_{0}^{\prime \prime}=\{6,7,8\}$. Então $Q^{\prime}$ e $Q^{\prime \prime}$ são aljavas de tipo $\mathbb{A}_{4}$ com único poço em 3 e $\mathbb{A}_{3}$ com único poço em 6 , respectivamente. Segue que $M$ é injetivo em $k Q^{\prime}$ ou injetivo em $k Q^{\prime \prime}$. Em qualquer caso, $M$ é injetivo em $k Q$.

\section{Poço em 6}

Temos as seguintes desigualdades: $x_{6} \leq x_{7} \leq x_{8} \leq 1, x_{6} \leq x_{5} \leq x_{3} \leq x_{2} \leq x_{1}$ e $x_{3} \leq x_{4}$.

Se $x_{6}=1$ então $x_{6}=x_{7}=x_{8}=1$. Em $120 q_{Q}\left(x_{1}, \ldots, x_{n}\right)=120$ temos $3\left(5-4 x_{5}\right)^{2}+$ $30\left(2 x_{1}-x_{2}\right)^{2}+2\left(6 x_{5}-5 x_{3}\right)^{2}+10\left(3 x_{2}-2 x_{3}\right)^{2}+30\left(2 x_{4}-x_{3}\right)^{2}=75$. De $3\left(5-4 x_{5}\right)^{2} \leq 75$ segue que $x_{5} \leq 2$. Se $x_{5}=2$ então teríamos $x_{2}=2$ e daí $x_{5}=1$, que é uma contradição pois $x_{5} \leq x_{2}$. Logo, $x_{5}=1$. Disto segue que $x_{2}=x_{3}=x_{4}=1 \mathrm{e}$, portanto, $M=I(6)$.

Se $x_{6}=0$ então $M$ é um $k Q^{\prime}$-módulo ou um $k Q^{\prime \prime}$-módulo onde $Q^{\prime}$ e $Q^{\prime \prime}$ são subaljavas plenas de $Q$ cujos conjuntos de vértices são $Q_{0}^{\prime}=\{7,8\}$ e $Q_{0}^{\prime \prime}=\{1,2,3,4,5\}$. Então $Q^{\prime}$ e $Q^{\prime \prime}$ são aljavas de tipo $\mathbb{A}_{2}$ com único poço em 7 e $\mathbb{D}_{5}$ com único poço em 5 , respectivamente. Segue que $M$ é injetivo em $k Q^{\prime}$ ou injetivo em $k Q^{\prime \prime}$. Em qualquer caso, $M$ é injetivo em $k Q$.

\section{Poço em 7}

Temos as seguintes desigualdades: $x_{7} \leq x_{6} \leq x_{5} \leq x_{3} \leq x_{2} \leq x_{1}, x_{3} \leq x_{4}$ e $x_{7} \leq x_{8} \leq 1$.

Se $x_{7}=1$ então $x_{8}=1$. Em $120 q_{Q}\left(x_{1}, \ldots, x_{n}\right)=120$ temos $5\left(4-3 x_{6}\right)^{2}+3\left(5 x_{6}-4 x_{5}\right)^{2}+$ $30\left(2 x_{1}-x_{2}\right)^{2}+2\left(6 x_{5}-5 x_{3}\right)^{2}+10\left(3 x_{2}-2 x_{3}\right)^{2}+30\left(2 x_{4}-x_{3}\right)^{2}=80$. Então de $5\left(4-3 x_{6}\right)^{2} \leq 80$ temos que $x_{6} \leq 2$.

- Se $x_{6}=2$ temos $3\left(10-4 x_{5}\right)^{2}+30\left(2 x_{1}-x_{2}\right)^{2}+2\left(6 x_{5}-5 x_{3}\right)^{2}+10\left(3 x_{2}-2 x_{3}\right)^{2}+30\left(2 x_{4}-\right.$ $\left.x_{3}\right)^{2}=60$. De $3\left(10-4 x_{5}\right)^{2} \leq 60$ e $x_{5} \geq x_{6}$ temos $x_{5}=2$ ou $x_{5}=3$. 
- Se $x_{5}=3$ então $30\left(2 x_{1}-x_{2}\right)^{2}+2\left(18-5 x_{3}\right)^{2}+10\left(3 x_{2}-2 x_{3}\right)^{2}+30\left(2 x_{4}-x_{3}\right)^{2}=48$. Daí $2\left(18-5 x_{3}\right)^{2} \leq 48$ e, portanto, $x_{3}=3$ ou $x_{3}=4$. Em qualquer caso teríamos $2\left(18-5 x_{3}\right)^{2} \geq 8$ e, portanto, $\left(2 x_{4}-x_{3}\right)^{2} \leq 1$, que é uma contradição.

- Se $x_{5}=2$ então $30\left(2 x_{1}-x_{2}\right)^{2}+2\left(12-5 x_{3}\right)^{2}+10\left(3 x_{2}-2 x_{3}\right)^{2}+30\left(2 x_{4}-x_{3}\right)^{2}=$ 48. De $2\left(12-5 x_{3}\right)^{2} \leq 48$ segue que $x_{3}=2$ ou $x_{3}=3$. Novamente, teríamos $2\left(18-5 x_{3}\right)^{2} \geq 8$ e, portanto, $\left(2 x_{4}-x_{3}\right)^{2} \leq 1$, que é uma contradição.

Portanto, $x_{6}=1$. Agora, $3\left(5-4 x_{5}\right)^{2}+30\left(2 x_{1}-x_{2}\right)^{2}+2\left(6 x_{5}-5 x_{3}\right)^{2}+10\left(3 x_{2}-2 x_{3}\right)^{2}+$ $30\left(2 x_{4}-x_{3}\right)^{2}=75$. De $3\left(5-4 x_{5}\right)^{2} \leq 75$ segue $x_{5} \leq 2$.

- Se $x_{5}=2$ então $30\left(2 x_{1}-x_{2}\right)^{2}+2\left(12-5 x_{3}\right)^{2}+10\left(3 x_{2}-2 x_{3}\right)^{2}+30\left(2 x_{4}-x_{3}\right)^{2}=48$ e então $2\left(12-5 x_{3}\right)^{2} \leq 48$, o que implica em $x_{3}=2$ ou $x_{3}=3$.

- Se $x_{3}=3$ então $30\left(2 x_{1}-x_{2}\right)^{2}+10\left(3 x_{2}-6\right)^{2}+30\left(2 x_{4}-3\right)^{2}=30$ e então $\left(2 x_{4}-3\right)^{2}=1$, que é uma contradição pois $x_{4} \geq x_{3}=3$.

- Se $x_{3}=2$ então $30\left(2 x_{1}-x_{2}\right)^{2}+10\left(3 x_{2}-4\right)^{2}+30\left(2 x_{4}-2\right)^{2}=40$ e então $3\left(2 x_{4}-2\right)^{2} \leq 4$, que é uma contradição pois $x_{4} \geq x_{3}=2$.

Portanto, $x_{5}=1$ e $30\left(2 x_{1}-x_{2}\right)^{2}+2\left(6-5 x_{3}\right)^{2}+10\left(3 x_{2}-2 x_{3}\right)^{2}+30\left(2 x_{4}-x_{3}\right)^{2}=72$. De $2\left(6-5 x_{3}\right)^{2} \leq 72$ segue que $x_{3} \leq 2$. Se $x_{3}=2$ então $30\left(2 x_{4}-2\right)^{2} \leq 40$, que é uma contradição pois $x_{4} \geq x_{3}$. Logo, $x_{3}=1$. Segue que $x_{4}=x_{2}=x_{1}=1$ e, portanto, $M=I(7)$.

Se $x_{7}=0$ então $M=S(8)=I(8)$ ou $M$ é um $k Q^{\prime}$-módulo onde $Q^{\prime}$ é a subaljava plena de $Q$ cujo conjunto de vértices é $Q_{0}^{\prime}=\{1,2,3,4,5,6\}$, que é uma aljava de tipo $\mathbb{E}_{6}$ com único poço em 6 . Segue então que $M$ é um $k Q^{\prime}$-módulo injetivo e, portanto, um $k Q$-módulo injetivo.

\section{Poço em 8}

Temos as seguintes desigualdades: $x_{8} \leq x_{7} \leq x_{6} \leq x_{3} \leq x_{2} \leq x_{1}$ e $x_{3} \leq x_{4}$.

Se $x_{8}=1$ então $10\left(3-2 x_{7}\right)^{2}+5\left(4 x_{7}-3 x_{6}\right)^{2}+3\left(5 x_{6}-4 x_{5}\right)^{2}+30\left(2 x_{1}-x_{2}\right)^{2}+2\left(6 x_{5}-\right.$ $\left.5 x_{3}\right)^{2}+10\left(3 x_{2}-2 x_{3}\right)^{2}+30\left(2 x_{4}-x_{3}\right)^{2}=90$. De $10\left(3-2 x_{7}\right)^{2} \leq 90$ segue que $x_{7} \leq 3$.

- Se $x_{7}=3$ então $5\left(12-3 x_{6}\right)^{2}+3\left(5 x_{6}-4 x_{5}\right)^{2}+30\left(2 x_{1}-x_{2}\right)^{2}+2\left(6 x_{5}-5 x_{3}\right)^{2}+10\left(3 x_{2}-\right.$ $\left.2 x_{3}\right)^{2}+30\left(2 x_{4}-x_{3}\right)^{2}=0$ e, portanto, $x_{3}=6$ e $x_{2}=4$, que é uma contradição pois $x_{3} \leq x_{2}$.

- Se $x_{7}=2$ então $5\left(8-3 x_{6}\right)^{2}+3\left(5 x_{6}-4 x_{5}\right)^{2}+30\left(2 x_{1}-x_{2}\right)^{2}+2\left(6 x_{5}-5 x_{3}\right)^{2}+10\left(3 x_{2}-\right.$ $\left.2 x_{3}\right)^{2}+30\left(2 x_{4}-x_{3}\right)^{2}=80$. De $5\left(8-3 x_{6}\right)^{2} \leq 80$ e $x_{6} \geq x_{7}$ segue que $2 \leq x_{6} \leq 4$.

- Se $x_{6}=4$ então $3\left(20-4 x_{5}\right)^{2}+30\left(2 x_{1}-x_{2}\right)^{2}+2\left(6 x_{5}-5 x_{3}\right)^{2}+10\left(3 x_{2}-2 x_{3}\right)^{2}+$ $30\left(2 x_{4}-x_{3}\right)^{2}=0$. Segue que $x_{3}=6$ e $x_{2}=4$, que é uma contradição.

- Se $x_{6}=3$ então $3\left(15-4 x_{5}\right)^{2}+30\left(2 x_{1}-x_{2}\right)^{2}+2\left(6 x_{5}-5 x_{3}\right)^{2}+10\left(3 x_{2}-2 x_{3}\right)^{2}+$ $30\left(2 x_{4}-x_{3}\right)^{2}=75$. De $3\left(15-4 x_{5}\right)^{2} \leq 75$ temos que $3 \leq x_{5} \leq 5$. Vejamos que não pode ocorrer nenhum dos casos. 
$*$ Se $x_{5}=5$ então $30\left(2 x_{1}-x_{2}\right)^{2}+2\left(30-5 x_{3}\right)^{2}+10\left(3 x_{2}-2 x_{3}\right)^{2}+30\left(2 x_{4}-x_{3}\right)^{2}=0$ e daí $x_{3}=6$ e $x_{2}=4$, que é uma contradição.

* Se $x_{5}=4$ então $30\left(2 x_{1}-x_{2}\right)^{2}+2\left(24-5 x_{3}\right)^{2}+10\left(3 x_{2}-2 x_{3}\right)^{2}+30\left(2 x_{4}-x_{3}\right)^{2}=$ 72 . De $2\left(24-5 x_{3}\right)^{2} \leq 72$ temos que $4 \leq x_{3} \leq 6$. Vejamos que nenhum desses casos ocorre.

- Se $x_{3}=6$ então $x_{2}=4$ contradizendo $x_{3} \leq x_{2}$.

- Se $x_{3}=5$ então $30\left(2 x_{1}-x_{2}\right)^{2}+10\left(3 x_{2}-10\right)^{2}+30\left(2 x_{4}-5\right)^{2}=70$. Daí $10\left(3 x_{2}-10\right)^{2} \leq 70$ que é uma contradição pois $x_{2} \geq 5$.

- Se $x_{3}=4$ então $30\left(2 x_{1}-x_{2}\right)^{2}+10\left(3 x_{2}-8\right)^{2}+30\left(2 x_{4}-4\right)^{2}=40$. Daí $\left(3 x_{2}-8\right)^{2} \leq 4$ que é uma contradição pois $x_{2} \geq 5$.

Portanto, $x_{5} \neq 4$.

* Se $x_{5}=3$ então $30\left(2 x_{1}-x_{2}\right)^{2}+2\left(18-5 x_{3}\right)^{2}+10\left(3 x_{2}-2 x_{3}\right)^{2}+30\left(2 x_{4}-x_{3}\right)^{2}=$ 48. De $2\left(18-5 x_{3}\right)^{2} \leq 48$ temos que $x_{3}=3$ ou $x_{3}=4$.

- Se $x_{3}=4$ então $30\left(2 x_{4}-4\right)^{2} \leq 40$, que é uma contradição pois $x_{4} \geq 4$.

- Se $x_{3}=3$ então $30\left(2 x_{4}-3\right)^{2} \leq 30$, que é uma contradição pois $x_{4} \geq 3$.

- Se $x_{6}=2$ então $3\left(10-4 x_{5}\right)^{2}+30\left(2 x_{1}-x_{2}\right)^{2}+2\left(6 x_{5}-5 x_{3}\right)^{2}+10\left(3 x_{2}-2 x_{3}\right)^{2}+$ $30\left(2 x_{4}-x_{3}\right)^{2}=60$. De $3\left(10-4 x_{5}\right)^{2} \leq 60$ segue que $x_{5}=2$ ou $x_{5}=3$.

$*$ Se $x_{5}=3$ então $30\left(2 x_{1}-x_{2}\right)^{2}+2\left(18-5 x_{3}\right)^{2}+10\left(3 x_{2}-2 x_{3}\right)^{2}+30\left(2 x_{4}-x_{3}\right)^{2}=$ 48. De $2\left(18-5 x_{3}\right)^{2} \leq 48$ temos que $x_{3}=3$ ou $x_{3}=4$.

- Se $x_{3}=4$ então $30\left(2 x_{1}-x_{2}\right)^{2}+10\left(3 x_{2}-4\right)^{2}+30\left(2 x_{4}-4\right)^{2}=40$, o que nos leva a uma contradição.

- Se $x_{3}=3$ então $30\left(2 x_{1}-x_{2}\right)^{2}+10\left(3 x_{2}-2 x_{3}\right)^{2}+30\left(2 x_{4}-x_{3}\right)^{2}=30$,que também nos leva a uma contradição.

* Se $x_{5}=2$ então $30\left(2 x_{1}-x_{2}\right)^{2}+2\left(12-5 x_{3}\right)^{2}+10\left(3 x_{2}-2 x_{3}\right)^{2}+30\left(2 x_{4}-x_{3}\right)^{2}=$ 48. De $2\left(12-5 x_{3}\right)^{2} \leq 48$ segue que $x_{3}=2$ ou $x_{3}=3$.

- Se $x_{3}=3$ então $30\left(2 x_{1}-x_{2}\right)^{2}+10\left(3 x_{2}-6\right)^{2}+30\left(2 x_{4}-3\right)^{2}=30$. Logo, $\left(2 x_{4}-3\right)^{2} \leq 1$, o que nos leva a uma contradição pois $x_{4} \geq 3$.

- Se $x_{3}=2$ então $\left(2 x_{4}-2\right)^{2} \leq 1$, que também nos leva a uma contradição.

Portanto, $x_{7}=1$. Lembrando que até o momento temos apenas $x_{8}=x_{7}=1$. Substituindo em $120 q_{Q}\left(x_{1}, \ldots, x_{n}\right)=120$ temos $5\left(4-3 x_{6}\right)^{2}+3\left(5 x_{6}-4 x_{5}\right)^{2}+30\left(2 x_{1}-x_{2}\right)^{2}+2\left(6 x_{5}-5 x_{3}\right)^{2}+$ $10\left(3 x_{2}-2 x_{3}\right)^{2}+30\left(2 x_{4}-x_{3}\right)^{2}=80$. De $5\left(4-3 x_{6}\right)^{2} \leq 80$ temos que $x_{6} \leq 2$. Continuando o processo concluímos que $x_{6}=x_{5}=x_{4}=x_{3}=x_{2}=x_{1}=1$ e, portanto, $M=I(8)$.

Finalmente, se $x_{8}=0$ então $M$ é um $k Q^{\prime}$-módulo onde $Q^{\prime}$ é a subaljava plena de $Q$ cujo conjunto de vértices é $Q_{0}^{\prime}=\{1,2,3,4,5,6,7\}$ que é uma aljava de tipo $\mathbb{E}_{7}$ com único poço em 7. Segue então que $M$ é um $k Q^{\prime}$-módulo injetivo e, portanto, um $k Q$-módulo injetivo.

Com esse, terminamos todos os casos possíveis para aljavas de tipo Dynkin com um único poço e, portanto, concluímos a prova. 
Segundo observou o Professor Viktor Bekkert na ocasião da defesa, o mesmo resultado também pode ser obtido com uma prova mais curta utilizando indução e o fato de que uma aljava de tipo Dynkin é uma árvore. 


\section{Capítulo 3}

\section{Produto fibrado de álgebras}

Neste capítulo iremos destacar propriedades que essa construção possui quando tratamos de álgebras e, ainda mais especificamente, álgebras básicas de dimensão finita sobre um corpo algebricamente fechado, para as quais o Teorema de Gabriel (Teorema 1.1.5) garante a existência de aljavas com relações que as determina.

A partir da Seção 3.2 nos restringimos a casos especiais em que temos certo controle sobre as aljavas ordinárias das álgebras envolvidas, o que nos permite chegar a diversos resultados. O principal tipo de produto fibrado aqui estudado é o chamado produto fibrado orientado. Alguns tipos de produto fibrado orientado foram estudados em [IPTZ87] e em [Lev04]. Na Seção 3.3.1, definimos o produto fibrado Dynkin orientado que generaliza ambos e a partir do resultado mostrado no capítulo precedente conseguimos uma generalização para [IPTZ87](3.5), mostrando que os módulos sobre esse tipo de produto fibrado se restringem aos módulos já existentes sobre as álgebras envolvidas.

Além disso, para o produto fibrado orientado, estudamos algumas subcategorias especiais da categoria de módulos indecomponíveis e através delas conseguimos, por exemplo, generalizar [Lev08](2.4.7), mostrando que, sob certas condições, o produto fibrado de álgebras hereditárias é uma álgebra inclinada.

Fixemos $k$ um corpo algebricamente fechado. Além disso, por todo o capítulo, $A, B, C$, $S$ e $R$ denotam álgebras com unidade e de dimensão finita sobre $k$ e todos os módulos são finitamente gerados à direita.

\subsection{Produto fibrado de álgebras e de módulos}

Começaremos com a definição geral de produto fibrado em uma categoria.

Definição 3.1.1 Sejam $\mathcal{C}$ uma categoria e dois morfismos $f_{1}: X_{1} \rightarrow X$ e $f_{2}: X_{2} \rightarrow X$ com $X, X_{1}$ e $X_{2}$ objetos de $\mathcal{C}$. Um produto fibrado do par $\left(f_{1}, f_{2}\right)$ é uma tripla $\left(W, h_{1}, h_{2}\right)$ onde $W$ é um objeto de $\mathcal{C}$ e $h_{1}: W \rightarrow X_{1}, h_{2}: W \rightarrow X_{2}$ são morfismos em $\mathcal{C}$ tais que:

(i) $f_{1} h_{1}=f_{2} h_{2} e$

(ii) para toda objeto $Z$ de $\mathcal{C}$ e todo par de morfismos $g_{1}: Z \rightarrow X_{1}$ e $g_{2}: Z \rightarrow X_{2}$ tal que $f_{1} g_{1}=f_{2} g_{2}$, existe um único morfimo $g: Z \rightarrow W$ tal que $h_{1} g=g_{1}$ e $h_{2} g=g_{2}$. 


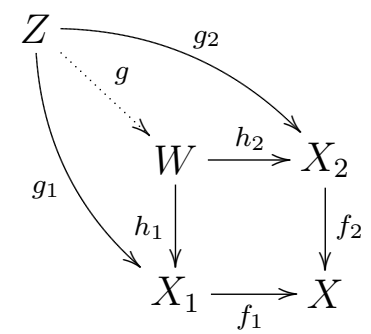

Segue da propriedade (ii) da definição que quando existe um produto fibrado, $\left(W, h_{1}, h_{2}\right)$ do par $\left(f_{1}, f_{2}\right)$, este é único a menos de isomorfismos, ou seja, se $\left(W^{\prime}, h_{1}^{\prime}, h_{2}^{\prime}\right)$ também for um produto fibrado do par $\left(f_{1}, f_{2}\right)$ então existe um isomorfismo $g: W \rightarrow W^{\prime}$ tal que $h_{1}=h_{1}^{\prime} g$ e $h_{2}=h_{2}^{\prime} g$. Por simplicidade, quando não houver ambiguidade, diremos apenas que o objeto $W$ é o produto fibrado de $f_{1}$ e $f_{2}$. Diremos nesse caso também, que o diagrama

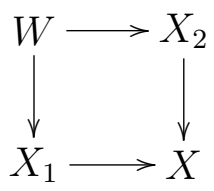

é um produto fibrado.

O produto fibrado comuta com somas diretas, ou seja, se

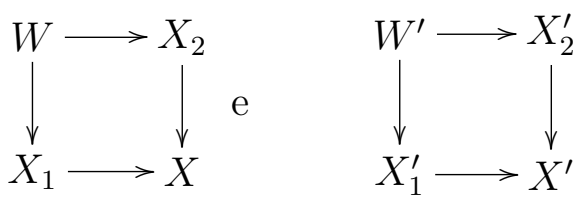

são produtos fibrados então

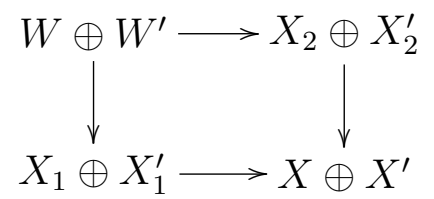

é também um produto fibrado. Tal propriedade pode ser encontrada, por exemplo, em [Rot79] (ver limites inversos).

Dados os morfismos de módulos $f_{1}: X_{1} \rightarrow X$ e $f_{2}: X_{2} \rightarrow X$, sempre existe o produto fibrado de $\left(f_{1}, f_{2}\right)$. Na verdade, é fácil mostrar que tal produto fibrado $\left(W, h_{1}, h_{2}\right)$ pode ser descrito como sendo o seguinte submódulo de $X_{1} \times X_{2}$ :

$$
W=\left\{\left(x_{1}, x_{2}\right) \in X_{1} \times X_{2} \mid f_{1}\left(x_{1}\right)=f_{2}\left(x_{2}\right)\right\}
$$

onde $h_{1}$ e $h_{2}$ são as restrições das projeções canônicas de $X_{1} \times X_{2}$ a $X_{1}$ e a $X_{2}$, respectivamente.

O mesmo resultado é válido para álgebras, ou seja, dados os morfismos de álgebras $f_{A}: A \rightarrow B$ e $f_{C}: C \rightarrow B$, o produto fibrado $\left(R, h_{A}, h_{C}\right)$ do par $\left(f_{A}, f_{C}\right)$ é a subálgebra de 
$A \times C$ dada por

$$
R=\left\{(a, c) \in A \times C \mid f_{A}(a)=f_{C}(c)\right\}
$$

e $h_{A}$ e $h_{C}$ são as restrições das projeções canônicas de $A \times C$ a $A$ e a $C$, respectivamente.

O próximo lema nos dá uma forma de verificar quando um diagrama comutativo

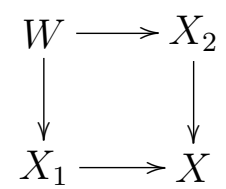

é um produto fibrado, ou seja, quando uma tripla $\left(W, h_{1}, h_{2}\right)$ que satisfaz (i) da Definição 3.1.1 é o produto fibrado de $f_{1}$ e $f_{2}$.

Enunciados semelhantes podem ser encontrados para categorias abelianas, como por exemplo [Ass97] (III.5.5). Faremos aqui, uma demonstração apenas para salientar que argumentos análogos são válidos também para álgebras.

Lema 3.1.1 Considere o diagrama comutativo de módulos

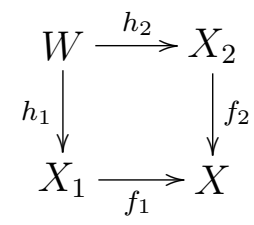

com $h_{2}$ um epimorfismo. Então, $\left(W, h_{1}, h_{2}\right)$ é o produto fibrado de $f_{1}$ e $f_{2}$ se, e somente se, a restrição de $h_{1}$ ao núcleo de $h_{2}$ é um isomorfismo sobre $\operatorname{ker} f_{1}$, isto é, $\left.h_{1}\right|_{\operatorname{ker} h_{2}}: \operatorname{ker} h_{2} \rightarrow \operatorname{ker} f_{1}$ é um isomorfismo.

Prova. Se $\left(W, h_{1}, h_{2}\right)$ é o produto fibrado de $f_{1}$ e $f_{2}$ então podemos considerar $W=$ $\left\{\left(x_{1}, x_{2}\right) \in X_{1} \times X_{2} \mid f_{1}\left(x_{1}\right)=f_{2}\left(x_{2}\right)\right\}$ com $h_{1}$ e $h_{2}$ as restrições das projeções canônicas de $X_{1} \times X_{2}$ a $X_{1}$ e a $X_{2}$, respectivamente.

Notemos, primeiramente, que $\operatorname{ker} h_{2}=\operatorname{ker} f_{1} \times 0$. Logo, é fácil ver que a restrição de $h_{1}$ ao núcleo de $h_{2}$ é de fato uma bijeção.

Para a recíproca, denotemos o produto fibrado de $f_{1}$ e $f_{2}$ por

$$
Y=\left\{\left(x_{1}, x_{2}\right) \in X_{1} \times X_{2} \mid f_{1}\left(x_{1}\right)=f_{2}\left(x_{2}\right)\right\}
$$

com $p_{1}: Y \rightarrow X_{1}$ e $p_{2}: Y \rightarrow X_{2}$ as restrições das projeções. Pelo item (ii) da Definição 3.1.1 existe um morfismo $h: W \rightarrow Y$ tal que $p_{1} h=h_{1}$ e $p_{2} h=h_{2}$. Basta agora mostrar que $h$ é um isomorfismo. Seja $w$ em $W$ tal que $h(w)=(0,0)$. Daí $h_{2}(w)=p_{2} h(w)=0$ e portanto $w$ está no núcleo de $h_{2}$. Além disso, $h_{1}(w)=p_{1} h(w)=0$ e como, por hipótese, $\left.h_{1}\right|_{\text {ker } h_{2}}$ é uma bijeção segue que $w=0$ e portanto $h$ é um monomorfismo. Para ver que $h$ é também um epimorfismo, considere $x=\left(x_{1}, x_{2}\right)$ em $Y$. Como $x_{2} \in X_{2}$ e $h_{2}$ é um epimorfismo então existe $z \in W$ tal que $h_{2}(z)=x_{2}$. Como $f_{1}\left(x_{1}\right)=f_{2}\left(x_{2}\right)$ e $f_{1} h_{1}=f_{2} h_{2}$ temos que $h_{1}(z)-x_{1}$ 
está em $\operatorname{ker} f_{1}$ pois

$$
f_{1}\left(h_{1}(z)-x_{1}\right)=f_{1} h_{1}(z)-f_{1}\left(x_{1}\right)=f_{2} h_{2}(z)-f_{2}\left(x_{2}\right)=f_{2}\left(x_{2}\right)-f_{2}\left(x_{2}\right)=0 .
$$

Portanto, existe $y \in \operatorname{ker} h_{2}$ tal que $h_{1}(y)=h_{1}(z)-x_{1}$ e segue que $h_{1}(z-y)=x_{1}$. Finalmente, como $h_{2}(z-y)=x_{2}$ segue que $h(z-y)=x$.

Note que argumentos análogos são válidos para álgebras e portanto podemos enunciar um resultado para estas.

Lema 3.1.2 Considere o diagrama comutativo de álgebras

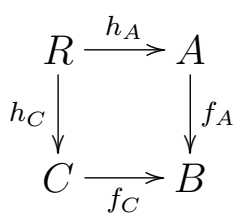

com $h_{A}$ um epimorfismo. Então, $\left(R, h_{A}, h_{C}\right)$ é o produto fibrado de $f_{A}$ e $f_{C}$ se, e somente se, a restrição de $h_{C}$ ao núcleo de $h_{A}$ é uma bijeção entre $\operatorname{ker} h_{A}$ e $\operatorname{ker} f_{C}$, isto é, $\left.h_{C}\right|_{\operatorname{ker} h_{A}}: \operatorname{ker} h_{A} \rightarrow \operatorname{ker} f_{C}$ é uma bijeção.

Consideremos agora o caso em que $f_{A}: A \rightarrow B$ e $f_{C}: C \rightarrow B$ são epimorfismos de álgebras e seja $\left(R, h_{A}, h_{C}\right)$ o produto fibrado de $f_{A}$ e $f_{C}$, ou seja, $R=\left\{(a, c) \in A \times C \mid f_{A}(a)=f_{C}(c)\right\}$ e $h_{A}$ e $h_{C}$ são as restrições das projeções. Denotemos o núcleo de $h_{A}$ por $\mathbb{I}_{A}$ e o núcleo de $h_{C}$ por $\mathbb{I}_{C}$.

A imagem de $h_{A}$ é $h_{A}(R)=\{a \in A \mid(a, c) \in R\}=\left\{a \in A \mid\right.$ existe $c \in C \operatorname{com} f_{C}(c)=$ $\left.f_{A}(a)\right\}$. Como $f_{C}$ é um epimorfismo, segue que $h_{A}(R)=A$ e portanto $h_{A}$ é também um epimorfismo. Analogamente, como $f_{A}$ é um epimorfismo, temos que $h_{C}$ também o será. Segue que $A \cong R / \mathbb{I}_{A}$ e $C \cong R / \mathbb{I}_{C}$. Além disso, temos que $f_{A} h_{A}=f_{C} h_{C}$ é também um epimorfismo e portanto $B \cong R / \operatorname{ker} f_{A} h_{A}$.

Vejamos que $\operatorname{ker} f_{A} h_{A}=\operatorname{ker} f_{C} h_{C}$ é $\mathbb{I}_{A}+\mathbb{I}_{C}$.

De fato, do lema 3.1.2 temos que $h_{C}{\mid \mathbb{I}_{A}}_{A}: \mathbb{I}_{A} \rightarrow \operatorname{ker} f_{C}$ e $\left.h_{A}\right|_{\mathbb{I}_{C}}: \mathbb{I}_{C} \rightarrow \operatorname{ker} f_{A}$ são bijeções e disto segue a sequência de igualdades:

$$
\begin{aligned}
\operatorname{ker} f_{A} h_{A} & =\left\{(a, c) \in R \mid f_{A} h_{A}(a, c)=0=f_{C} h_{C}(a, c)\right\} \\
& =\left\{(a, c) \in R \mid f_{A}(a)=0=f_{C}(c)\right\} \\
& =\left\{(a, c) \in R \mid a \in \operatorname{ker} f_{A} \text { e } c \in \operatorname{ker} f_{C}\right\} \\
& =\left\{(a, c) \in R \mid(a, 0) \in \mathbb{I}_{C} \text { e }(0, c) \in \mathbb{I}_{A}\right\} \\
& =\mathbb{I}_{A}+\mathbb{I}_{C} .
\end{aligned}
$$


Como $h_{A}$ e $h_{C}$ são as restrições das projeções então é fácil verificar que $\mathbb{I}_{A} \cap \mathbb{I}_{C}=0$ e, portanto, que $\operatorname{ker} f_{A} h_{A}$ é igual a $\mathbb{I}_{A} \oplus \mathbb{I}_{C}$. Logo, $B \cong R /\left(\mathbb{I}_{A} \oplus \mathbb{I}_{C}\right)$.

O próximo resultado pode ser encontrado em [Pog01] (2.1), mas faremos aqui uma prova utilizando o Lema 3.1.2.

Proposição 3.1.3 Sejam $R$ uma álgebra, $\mathbb{I}_{A}$ e $\mathbb{I}_{C}$ ideais de $R$. O diagrama comutativo de álgebras

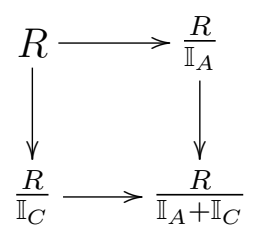

onde os morfismos são os respectivos epimorfismos naturais, é um produto fibrado se, e somente se, $\mathbb{I}_{A} \cap \mathbb{I}_{C}=0$.

Prova. A restrição à $\mathbb{I}_{A}$ do epimorfismo natural $R \rightarrow R / \mathbb{I}_{C}$ é uma bijeção entre $\mathbb{I}_{A}$ e $\left(\mathbb{I}_{A}+\mathbb{I}_{C}\right) / \mathbb{I}_{C}$ se, e somente se, $\mathbb{I}_{A} \cap \mathbb{I}_{C}=0$. O resultado segue então do Lema 3.1.2.

Veremos um mesmo exemplo em formas diferentes:

Exemplo 3.1.4 Considere $\left(\begin{array}{ll}k & 0 \\ k & k\end{array}\right)$ a álgebra das matrizes triangulares e o epimorfismo $f:\left(\begin{array}{ll}k & 0 \\ k & k\end{array}\right) \rightarrow k^{2}$ dada por $f\left(\begin{array}{ll}x & 0 \\ y & z\end{array}\right)=(x, z)$. Então o produto fibrado de $(f, f)$ é dado

$$
\begin{aligned}
&\left\{\left(\left(\begin{array}{ll}
x & 0 \\
y & z
\end{array}\right),\left(\begin{array}{ll}
r & 0 \\
s & t
\end{array}\right)\right) \mid(x, z)=(r, t), x, y, z, r, s, t \in k\right\}= \\
&\left\{\left(\left(\begin{array}{ll}
x & 0 \\
y & z
\end{array}\right),\left(\begin{array}{ll}
x & 0 \\
s & z
\end{array}\right)\right) \mid x, y, z, s \in k\right\} \cong \\
&\left\{\left(\begin{array}{cc}
x & 0 \\
(y, s) & z
\end{array}\right) \mid x, y, z, s \in k\right\}=\left(\begin{array}{cc}
k & 0 \\
k^{2} & k
\end{array}\right)
\end{aligned}
$$

que é a conhecida álgebra de Kronecker.

Exemplo 3.1.5 O exemplo anterior no contexto de álgebras de caminhos nos mostra que se $A=C$ é a álgebra de caminhos $k Q$ onde $Q$ é a aljava $\bullet \longleftarrow \bullet$ e $B$ é a álgebra semisimples cuja aljava é • $\quad$, então, o produto fibrado $R$ de $(f, f)$, onde $f: A=C \rightarrow B$ é o epimorfismo natural, é a álgebra de caminhos cuja aljava ordinária é $\bullet \longleftarrow \bullet$.

Exemplo 3.1.6 Uma outra forma de verificar o resultado desse exemplo no contexto de aljavas é utilizando a Proposição 3.1.3. Seguindo a notação do Exemplo 3.1.5, denotemos por $\alpha$ e $\beta$ as flechas da álgebra de Kronecker $R$. Então o núcleo do epimorfismo sobre $A$ e sobre $C$ é gerado por uma flecha, digamos $\mathbb{I}_{A}=\left\langle\alpha>e \mathbb{I}_{C}=\langle\beta>\right.$. Então $A \cong R /<\alpha>$, 
$C=R /<\beta>$ e $B=R /(<\alpha>+<\beta>)$. Como $\langle\alpha>\cap<\beta>=0$ segue que $R$ é o produto fibrado das aplicações naturais $A \rightarrow B$ e $C \rightarrow B$.

\subsubsection{Módulos sobre o produto fibrado}

Dado um morfismo de álgebras $\phi: \Lambda_{1} \rightarrow \Lambda_{2}$, podemos olhar um $\Lambda_{2}$-módulo $M$ como um $\Lambda_{1}$-módulo através do produto dado por $\lambda \cdot m=\phi(\lambda) m$, para $\lambda \in \Lambda_{1}$ e $m \in M$. Além disso, se $f: M \rightarrow N$ é um morfismo de $\Lambda_{2}$-módulos então $f$ é também um morfismo de $\Lambda_{1}$-módulos. Mais ainda, se $\phi$ é um epimorfismo e $g: M_{\Lambda_{2}} \rightarrow N_{\Lambda_{2}}$ é um morfismo de $\Lambda_{1}$-módulos então $g$ é também um morfismo de $\Lambda_{2}$-módulos. Ou seja, se $\phi$ é um epimorfismo então podemos olhar $\bmod \Lambda_{2}$ como uma subcategoria plena de $\bmod \Lambda_{1}$.

Consideremos a situação da Proposição 3.1.3, ou seja, $A=R / \mathbb{I}_{A}, C=R / \mathbb{I}_{C}, B=R /\left(\mathbb{I}_{A} \oplus\right.$ $\left.\mathbb{I}_{C}\right)$ e $\left(R, h_{A}, h_{C}\right)$ o produto fibrado dos epimorfismos naturais $f_{A}: A \rightarrow B$ e $f_{C}: C \rightarrow B$.

Dos epimorfismos $f_{A}$ e $f_{C}$ podemos considerar a categoria $\bmod B$ como subcategoria plena de $\bmod A$ e de $\bmod C$ e estas duas como subcategorias plenas de $\bmod R$, através dos epimorfismos $h_{A}$ e $h_{C}$.

Veremos em exemplos mais adiante que esses não são todos os módulos de $R$, ou seja, podem existir $R$-módulos que não são nem $A$-módulos e nem $C$-módulos. Por outro lado, o próximo resultado nos garante que, sob certas condições, todo $R$-módulo $M$ pode ser visto como um produto fibrado de morfismos da forma $X \rightarrow Z$ e $Y \rightarrow Z$ onde $X$ é um $A$-módulo, $Y$ é um $C$-módulo e $Z$ é um $B$-módulo. Para isso, notemos que se $M$ é um $R$-módulo então $M / M \mathbb{I}_{A}$ é um $A$-módulo, $M / M \mathbb{I}_{C}$ é um $C$-módulo e $M / M\left(\mathbb{I}_{A} \oplus \mathbb{I}_{C}\right)=M /\left(M \mathbb{I}_{A}+M \mathbb{I}_{C}\right)$ é um $B$-módulo.

Com isso, temos um resultado análogo à Proposição 3.1 .3 para módulos (ver também [Pog01] (2.3)).

\section{Proposição 3.1.7 Seja}

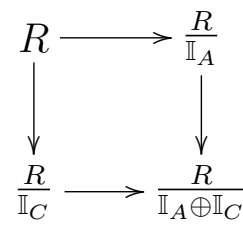
um produto fibrado de álgebras. Então o dia-

grama comutativo de $R$-módulos

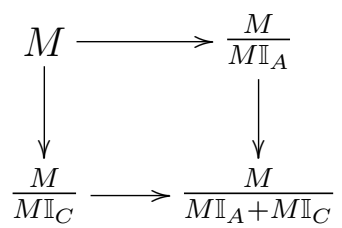

onde os morfismos são os epimorfismos naturais, é um produto fibrado de $R$-módulos se, e somente se, $M \mathbb{I}_{A} \cap M \mathbb{I}_{C}=0$.

Prova. Observemos que os morfismos são morfismos de $R$-módulos e que a restrição à $M \mathbb{I}_{C}$ do epimorfismo $M \rightarrow M / M \mathbb{I}_{C}$ é uma bijeção entre $M \mathbb{I}_{A}$ e $\left(M \mathbb{I}_{A}+M \mathbb{I}_{C}\right) / M \mathbb{I}_{C}$ se, e somente se, $M \mathbb{I}_{A} \cap M \mathbb{I}_{C}=0$. O resultado segue do Lema 3.1.1. 
É bem conhecido (por exemplo [Ass97](V.2.6)) que para uma álgebra $S$ e um ideal II de $S$ vale o seguinte isomorfismo de $S / \mathbb{I}$-módulos:

$$
M \otimes_{S} S / \mathbb{I} \cong M / M \mathbb{I} .
$$

No nosso caso, para $S=A, B$ ou $C$, tal isomorfismo é também de $R$-módulos e então podemos reenunciar a última proposição como segue:

Proposição 3.1.8 Seja $R$ o produto fibrado de $R / \mathbb{I}_{A} \rightarrow R /\left(\mathbb{I}_{A} \oplus \mathbb{I}_{C}\right)$ e $R / \mathbb{I}_{C} \rightarrow R /\left(\mathbb{I}_{A} \oplus \mathbb{I}_{C}\right)$. Um R-módulo $M$ é o produto fibrado de $M \otimes_{R} A \rightarrow M \otimes_{R} B$ e $M \otimes_{R} C \rightarrow M \otimes_{R} B$ se, e somente se, $M \mathbb{I}_{A} \cap M \mathbb{I}_{C}=0$.

Vejamos que para um $R$-módulo projetivo $P$, existem morfismos $f: X \rightarrow Z$ e $g: Y \rightarrow Z$ onde $X$ é um $A$-módulo projetivo, $Y$ é um $C$-módulo projetivo e $Z$ é um $B$-módulo projetivo, tais que $P$ é o produto fibrado de $(f, g)$.

Proposição 3.1.9 Seja $R$ o produto fibrado de $R / \mathbb{I}_{A} \rightarrow R /\left(\mathbb{I}_{A} \oplus \mathbb{I}_{C}\right)$ e $R / \mathbb{I}_{C} \rightarrow R /\left(\mathbb{I}_{A} \oplus \mathbb{I}_{C}\right)$. Cada $R$-módulo projetivo $P$ é o produto fibrado de $P \otimes_{R} A \rightarrow P \otimes_{R} B$ e $P \otimes_{R} C \rightarrow P \otimes_{R} B$.

Prova. Já vimos que $\mathbb{I}_{A} \cap \mathbb{I}_{C}=0$ e portanto, como $R \mathbb{I}_{A}=\mathbb{I}_{A}$ e $R \mathbb{I}_{C}=\mathbb{I}_{C}$, temos que $R \mathbb{I}_{A} \cap R \mathbb{I}_{C}=0$. Se $P$ é um $R$-módulo projetivo indecomponível temos $P \mathbb{I}_{A} \cap P \mathbb{I}_{C} \subseteq$ $R \mathbb{I}_{A} \cap R \mathbb{I}_{C}=0$. Pela proposição anterior segue que $P$ é o produto fibrado de $P \otimes_{R} A \rightarrow P \otimes_{R} B$ e $P \otimes_{R} C \rightarrow P \otimes_{R} B$. O resultado também é válido para qualquer projetivo pois produto fibrado comuta com somas diretas.

Para verificar se todo $R$-módulo pode ser escrito como um produto fibrado de módulos como acima, basta olharmos o que acontece com os $R$-módulos injetivos (Ver também $[\operatorname{Pog} 01](4.2))$.

Lema 3.1.10 Seja $R$ o produto fibrado de $R / \mathbb{I}_{A} \rightarrow R /\left(\mathbb{I}_{A} \oplus \mathbb{I}_{C}\right)$ e $R / \mathbb{I}_{C} \rightarrow R /\left(\mathbb{I}_{A} \oplus \mathbb{I}_{C}\right)$. Todo $R$-módulo $M$ é o produto fibrado de $M \otimes_{R} A \rightarrow M \otimes_{R} B$ e $M \otimes_{R} C \rightarrow M \otimes_{R} B$ se, e somente se, $I \mathbb{I}_{A} \cap I \mathbb{I}_{C}=0$ para todo $R$-módulo injetivo $I$.

Prova. Pela Proposição 3.1.8, basta ver que $M \mathbb{I}_{A} \cap M \mathbb{I}_{C}=0$ para todo $R$-módulo $M$ se, e somente se, $I \mathbb{I}_{A} \cap I \mathbb{I}_{C}=0$ para todo $R$-módulo injetivo $I$.

Seja $M$ um $R$-módulo e considere $\iota: M \rightarrow I(M)$ a envolvente injetiva de $M$. Temos que

$$
\iota\left(M \mathbb{I}_{A} \cap M \mathbb{I}_{C}\right) \subseteq \iota\left(M \mathbb{I}_{A}\right) \cap \iota\left(M \mathbb{I}_{C}\right)=\iota(M) \mathbb{I}_{A} \cap \iota(M) \mathbb{I}_{C} \subseteq I(M) \mathbb{I}_{A} \cap I(M) \mathbb{I}_{C} .
$$

Como $I(M) \mathbb{I}_{A} \cap I(M) \mathbb{I}_{C}=0$ segue que $\iota\left(M \mathbb{I}_{A} \cap M \mathbb{I}_{C}\right)=0$ e como $\iota$ é um monomorfismo então $M \mathbb{I}_{A} \cap M \mathbb{I}_{C}=0$. A outra implicação é óbvia. 


\subsubsection{Caso estudado: aljavas}

Para o resto desse trabalho, ao escrever que $R$ é o produto fibrado dos epimorfismos $A \rightarrow B$ e $C \rightarrow B$ estaremos considerando $A, B, C$ e $R$ como descritos nessa seção.

Como já dito, estamos interessados em estudar o produto fibrado de álgebras no contexto de aljavas, por isso, desde o início desse capítulo nossas álgebras são básicas e de dimensão finita sobre $k$. A partir de agora, e pelo resto desse capítulo, vamos considerar as álgebras da forma $S=k Q_{S} / I_{S}$ onde $Q_{S}$ é uma aljava e $I_{S}$ é um ideal admissível de $k Q_{S}$. Denotemos $e_{S}=\sum_{i \in\left(Q_{S}\right)_{0}} e_{i}$ que é a unidade da álgebra $S$.

Sejam $A=k Q_{A} / I_{A}$ e $C=k Q_{C} / I_{C}$ duas álgebras onde $Q_{A}$ e $Q_{C}$ são aljavas conexas e $I_{A}$ e $I_{C}$ são ideais admissíveis em $k Q_{A}$ e $k Q_{C}$, respectivamente. Seja também $Q_{B}$ uma subaljava plena e convexa (não necessariamente conexa) de $Q_{A}$ e de $Q_{C}$ e suponhamos que $I_{A} \cap k Q_{B}=I_{C} \cap k Q_{B}$, o que será denotado por $I_{B}$. Denotemos $k Q_{B} / I_{B}$ por $B$.

Lema 3.1.11 Nas condições acima temos que $B \cong e_{B} A e_{B} \cong e_{B} C e_{B}$.

Prova. Como $I_{B}$ está contido em $I_{A}$ podemos definir a seguinte aplicação $\Phi: B \rightarrow e_{B} A e_{B}$ dada por $\omega+I_{B} \mapsto \omega+I_{A}$, para $\omega$ um caminho em $Q_{B}$. Então, $\Phi$ é um morfismo de $k$-álgebras. Vejamos que se trata de um isomorfismo.

Seja $\omega$ em $k Q_{B}$ tal que $\omega+I_{A}$ é nulo. Então, $\omega$ está em $k Q_{B} \cap I_{A}$ que por definição é $I_{B}$, ou seja, $\omega+I_{B}=0$ e portanto a aplicação $\Phi$ é um monomorfismo. Vejamos que também é sobrejetora. Seja $\omega$ um caminho em $Q_{A}$. Se $e_{B} \omega e_{B}$ é nulo então o elemento nulo em $B$ é uma pré-imagem para $e_{B} \omega e_{B}+I_{A}$. Suponha que $e_{B} \omega e_{B}$ é não nulo. Neste caso, $s(\omega)$ e $e(\omega)$ estão em $\left(Q_{B}\right)_{0}$ e, pela convexidade de $Q_{B}$ em $Q_{A}$, segue que cada vértice que aparece em $\omega$ está ainda em $\left(Q_{B}\right)_{0}$. Pela plenitude, cada flecha que aparece em $\omega$ está em $\left(Q_{B}\right)_{1}$ e portanto $\omega$ é um caminho em $Q_{B}$. Logo, o elemento $\omega+I_{B}$ de $B$ é uma pré-imagem para $e_{B} \omega e_{B}+I_{A}$.

$\mathrm{O}$ isomorfismo entre $B$ e $e_{B} C e_{B}$ se mostra de forma análoga.

Do lema anterior vemos que $B$ é um quociente comum de $A$ e de $C$. Sejam $f_{A}: A \rightarrow B$ e $f_{C}: C \rightarrow B$ os epimorfismos naturais e denotemos $R$ o produto fibrado de $f_{A}$ e $f_{C}$. Podemos escrever $f_{A}(a)=e_{B} a e_{B}$ para $a \in A$ e $f_{C}(c)=e_{B} c e_{B}$ para $c \in C$ e, pelo que já foi discutido anteriormente, temos que $R$ é a subálgebra de $A \times C$ dada pelos pares $(a, c) \in A \times C$ tais que $e_{B} a e_{B}=e_{B} c e_{B}$.

No Exemplo 3.1.5 temos um exemplo em que $B$ não é isomorfa à $e_{B} A e_{B}$. Note que $Q_{B}$ não é plena em $Q_{A}$.

Exemplo 3.1.12 Considere A a k-álgebra com aljava ordinária $Q_{A}=1$ $B$ a algebra cuja aljava é $Q_{B}=\nwarrow_{2}^{1} \swarrow^{4}$. Neste caso $Q_{B}$ é uma subaljava plena de $Q_{A}$, 
mas não é convexa. Temos que $\operatorname{dim}_{k} B=6$ e $\operatorname{dim}_{k}\left(e_{B} A e_{B}\right)=7$ e portanto $B$ e e $e_{B} A e_{B}$ não são isomorfas.

\subsubsection{Aljava ordinária do produto fibrado}

Antes de descrever a aljava ordinária de um produto fibrado precisaremos da noção de soma amalgamada de aljavas, que é a noção dual de um produto fibrado. Em uma categoria $\mathcal{C}$, dados dois morfismos $f_{1}: X \rightarrow X_{1}$ e $f_{2}: X \rightarrow X_{2}$, uma soma amalgamada do par $\left(f_{1}, f_{2}\right)$ é uma tripla $\left(Z, q_{1}, q_{2}\right)$ onde $Z$ é um objeto de $\mathcal{C}$ e $h_{1}: X_{1} \rightarrow Z$ e $h_{2}: X_{2} \rightarrow Z$ são morfismos tais que: (i) $q_{1} f_{1}=q_{2} f_{2}$ e (ii) dados um objeto $Y$ e dois morfismos $g_{1}: X_{1} \rightarrow Y$ e $g_{2}: X_{2} \rightarrow Y$ tais que $g_{1} f_{1}=g_{2} f_{2}$, então existe um único morfismo $h: Z \rightarrow Y$ tal que $h q_{1}=g_{1}$ e $h q_{2}=g_{2}$.

Como nos interessará neste trabalho apenas um caso particular de soma amalgamada (o de aljavas), vamos utilizar uma construção conhecida para grafos (aljavas não orientadas) (Ver por exemplo [Ehr79]) e escritas no contexto de aljavas em [Lev04].

Consideremos $i: Q \rightarrow W$ e $j: Q \rightarrow V$ inclusões de aljavas, onde $Q$ é uma subaljava plena de $W$ e de $V$. Definimos a seguinte aljava que será denotada por $W \coprod_{Q} V$ :

1. Os vértices são aqueles de $W$ e aqueles de $V$ que não estão em $j(Q)$.

2. As flechas são as de $W_{1}$ e:

a) para $x$ e $y$ em $V_{0} \backslash j(Q)_{0}$, há uma flecha de $x$ para $y$ para cada flecha de $x$ para $y$ em $V$;

b) para $x$ em $V_{0} \backslash j(Q)_{0}$ e $y=i(z)$ para algum $z \in Q$, há uma flecha de $x$ para $y$ para cada flecha de $x$ para $j(x)$ em $V$;

c) para $x=i(z)$ com $z \in Q$ e $y$ em $V_{0} \backslash j(Q)_{0}$, há uma flecha de $x$ para $y$ para cada flecha de $j(z)$ para $y$ em $V$.

Proposição 3.1.13 ([Lev04] 1.1.4) Com as notações acima, a aljava $W \coprod_{Q} V$ é a soma amalgamada das aplicações $i$ e $j$.

Antes de continuar vejamos um exemplo dessa construção.

Exemplo 3.1.14 Consideremos as aljavas

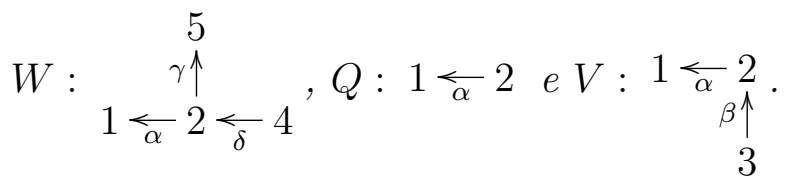

A soma amalgamada das inclusões $Q \rightarrow W$ e $Q \rightarrow V$ é $1<\frac{\alpha}{\gamma \uparrow} \underset{\beta}{2} \underset{\gamma}{\gamma} 4$ 
Passemos agora à descrição da aljava ordinária do produto fibrado $R$. Descrições análogas aparecem, por exemplo, em [IPTZ87] e [Lev04].

Consideremos novamente $R$ o produto fibrado dos epimorfismos de álgebras $A \rightarrow B$ e $C \rightarrow B$, onde $A, B$ e $C$ são álgebras de dimensão finita sobre $k$ como descritas no início da Seção 3.1.2.

Seja $Q$ a soma amalgamada das inclusões de aljavas $Q_{B} \rightarrow Q_{A}$ e $Q_{B} \rightarrow Q_{C}$. Notemos que nesse caso, os elementos de $Q$ que estão simultaneamente em $Q_{A}$ e em $Q_{C}$ são justamente os elementos de $Q_{B}$.

Suponha que $I$ é o ideal de $k Q$ gerado por $I_{A}, I_{C}$ e por todos os caminhos que ligam $\left(Q_{A}\right)_{0} \backslash\left(Q_{B}\right)_{0}$ e $\left(Q_{C}\right)_{0} \backslash\left(Q_{B}\right)_{0}$. Pela construção, vemos que $I_{A}=I \cap k Q_{A}$ e $I_{C}=I \cap k Q_{C}$. Vemos também que $\left(Q_{A}, I_{A}\right)$ e $\left(Q_{C}, I_{C}\right)$ são plenas e convexas em $(Q, I)$. Neste caso, temos que $A \cong e_{A}(k Q / I) e_{A}$ e $C \cong e_{C}(k Q / I) e_{C}$.

Denotemos $e_{C}-e_{B}$ por $e_{C}^{\prime}, e_{A}-e_{B}$ por $e_{A}^{\prime}$ e $e=e_{A}^{\prime}+e_{B}+e_{C}^{\prime}$ a identidade de $k Q / I$.

Para $S=A$ ou $C$, denotemos por $h_{S}: k Q / I \rightarrow e_{S}(k Q / I) e_{S}$ o epimorfismo dado por $h_{S}(x)=e_{S} x e_{S}$ para $x$ em $k Q / I$ e por $f_{S}: e_{S}(k Q / I) e_{S} \rightarrow e_{B}(k Q / I) e_{B}$ o epimorfismo dado por $f_{S}(x)=e_{B} x e_{B}$ para $x$ em $e_{S}(k Q / I) e_{S}$.

Temos o seguinte diagrama comutativo, visto que $e_{B} e_{A}=e_{B}=e_{B} e_{C}$

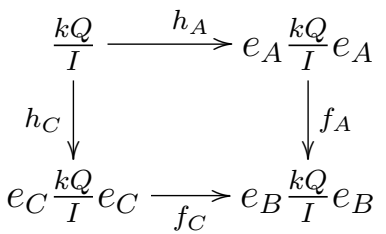

O núcleo de $h_{A}$ é dado por $\operatorname{ker} h_{A}=(k Q / I) e_{C}^{\prime}(k Q / I)$ e o núcleo de $f_{C}$ é $\operatorname{ker} f_{C}=$ $e_{C}(k Q / I) e_{C}^{\prime}(k Q / I) e_{C}$. Não é difícil ver que a restrição de $h_{C}$ ao núcleo de $h_{A}$ é uma bijeção sobre ker $f_{C}$ e, pelo Lema 3.1.2, segue que $k Q / I$ é o produto fibrado de $f_{A}$ e $f_{C}$.

Proposição 3.1.15 Sejam $R$ o produto fibrado dos epimorfismos $A \rightarrow B$ e $C \rightarrow B$, a aljava $Q$ a soma amalgamada das inclusões de aljavas $Q_{B} \rightarrow Q_{A}$ e $Q_{B} \rightarrow Q_{C}$ e $I$ o ideal de $k Q$ gerado por $I_{A}$, $I_{C}$ e por todos os caminhos que ligam $\left(Q_{A}\right)_{0} \backslash\left(Q_{B}\right)_{0}$ e $\left(Q_{C}\right)_{0} \backslash\left(Q_{B}\right)_{0}$. Então, o produto fibrado $R$ é isomorfo a $k Q / I$.

Outra forma de ver esse resultado é olhando para o seguinte diagrama comutativo, observando que o núcleo de $f_{A} h_{A}\left(=f_{C} h_{C}\right)$ é $(k Q / I)\left(e_{A}^{\prime}+e_{C}^{\prime}\right)(k Q / I)$,

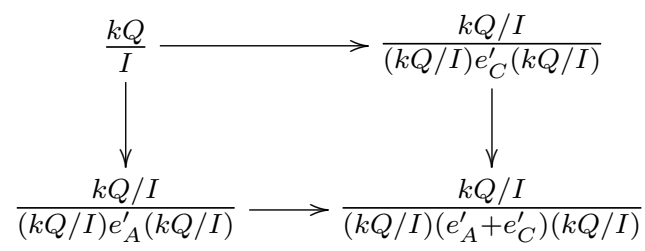


Pela Proposição 3.1.3, basta ver que

$$
(k Q / I) e_{C}^{\prime}(k Q / I) \cap(k Q / I) e_{A}^{\prime}(k Q / I)=0 .
$$

De fato, se $x$ está em $(k Q / I) e_{C}^{\prime}(k Q / I) \cap(k Q / I) e_{A}^{\prime}(k Q / I)$ então $x=e x e=e_{B} x e_{B}$ pois pela construção de $I$ temos que $e_{A}^{\prime}(k Q / I) e_{C}^{\prime}=e_{C}^{\prime}(k Q / I) e_{A}^{\prime}=0$. Mas, como $\left(Q_{B}, I_{B}\right)$ é convexa em $(Q, I)$, temos que $e_{B}(k Q / I) e_{C}^{\prime}(k Q / I) e_{B}=0=e_{B}(k Q / I) e_{A}^{\prime}(k Q / I) e_{B}$ e portanto $x=0$.

Exemplo 3.1.16 Sejam A, B e $C$ álgebras com as seguintes aljavas e relações:

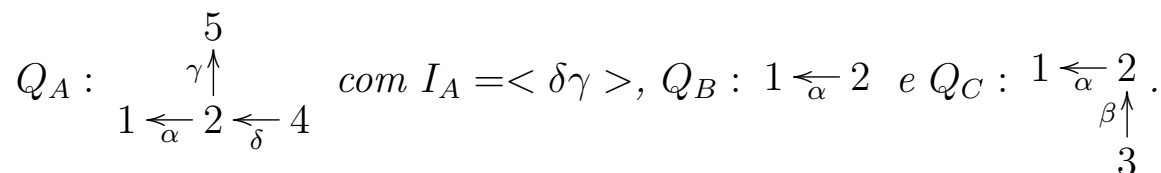

A aljava ordinária de $R$ é $Q: 1<\frac{\alpha}{\gamma \uparrow} \underset{\beta \uparrow}{2<} 4$ e $I=<\delta \gamma, \beta \gamma>$.

Por conveniência, usaremos as diferentes formas de olhar as álgebras envolvidas bem como seus módulos. Por exemplo, do epimorfismo $R \rightarrow A$ sabemos que todo $A$-módulo possui uma estrutura de $R$-módulo e podemos chegar a mesma conclusão a partir de representações dos módulos, visto que $\left(Q_{A}, I_{A}\right)$ é plena e convexa em $(Q, I)$. Faremos agora uma série de observações que serão úteis no decorrer desse trabalho. Aqui $S$ denota $A, B$ ou $C$.

- ind $S$ é uma subcategoria plena de ind $R$, bem $\operatorname{como}$ ind $B$ é uma subcategoria plena de ind $A$ e de $\operatorname{ind} C$.

- Para dois $S$-módulos $M$ e $N$ temos que $N$ é um $S$-submódulo de $M$ se e somente se $N$ for um $R$-submódulo de $M$. O mesmo vale para quocientes.

- Se $M$ é uma $S$-módulo e $N$ é um $R$-submódulo de $M$ então $N$ é também um $S$-módulo.

\subsection{Produto fibrado orientado}

A Proposição 3.1.7 nos dá uma condição para que um $R$ módulo $M$ seja o produto fibrado de epimorfismos da forma $M \otimes A \rightarrow M \otimes B$ e $M \otimes C \rightarrow M \otimes B$. Para que isso aconteça, neste caso, basta que $M R e_{C}^{\prime} R \cap M R e_{A}^{\prime} R=0$. Vejamos que essa intersecção está em $\bmod B$.

Lema 3.2.1 Seja $M$ um R-módulo, então $M \operatorname{Re}_{C}^{\prime} R \cap M \operatorname{Re}_{A}^{\prime} R=M \operatorname{Re}_{C}^{\prime} \operatorname{Re}_{B} \cap M \operatorname{Re}_{A}^{\prime} \operatorname{Re}_{B}$.

Prova. Seja $x$ em $M R e_{C}^{\prime} R \cap M R e_{A}^{\prime} R$. Basta mostrar que $x=x e_{B}$. Temos que $x=x e=$ $x e_{A}^{\prime}+x e_{B}+x e_{C}^{\prime}$. Mas $x e_{A}^{\prime} \in M R e_{C}^{\prime} R e_{A}^{\prime}=0$ pois $e_{C}^{\prime} R e_{A}^{\prime}=0$ e $x e_{C}^{\prime} \in M R e_{A}^{\prime} R e_{C}^{\prime}=0$ pois $e_{A}^{\prime} R e_{C}^{\prime}=0$. Portanto, $x=x e_{B}$ como queríamos. 
Pelo lema anterior, se $M$ é tal que $M R e_{C}^{\prime} R \cap M R e_{A}^{\prime} R \neq 0$ então existe $i$ em $\left(Q_{B}\right)_{0}$ e caminhos não nulos de $j$ para $i$, com $j$ em $\left(Q_{A}\right)_{0} \backslash\left(Q_{B}\right)_{0}$, e de $l$ para $i$, com $l$ em $\left(Q_{C}\right)_{0} \backslash\left(Q_{B}\right)_{0}$.

Exemplo 3.2.2 Considere as aljavas $Q_{A}=2 \stackrel{\alpha}{\rightarrow} 1, Q_{B}=1$ e $Q_{C}=1 \stackrel{\beta}{\leftarrow} 3$. Neste caso, o produto fibrado $R$ é dado por $Q_{R}=2 \stackrel{\alpha}{\rightarrow} 1 \stackrel{\beta}{\leftarrow} 3$. O injetivo associado ao vértice 1 é $I(1)=$ $k \stackrel{1}{\rightarrow} k \stackrel{1}{<} k$. Além disso, $e_{A}^{\prime}=e_{2}, e_{B}=e_{1}$ e $e_{C}^{\prime}=e_{3}$. Neste caso, I(1)R $e_{C}^{\prime} R \cap I(1) R e_{A}^{\prime} R \neq 0$ pois $I(1) \alpha=S(1)=I(1) \beta$.

Observe que a intersecção do Lema 3.2.1 será sempre nula para o produto fibrado definido abaixo.

Definição 3.2.1 Seja $R$ o produto fibrado dos epimorfismos $A \rightarrow B$ e $C \rightarrow B$. Dizemos que tal produto é orientado se não existir caminho não nulo de $\left(Q_{B}\right)_{0}$ para $\left(Q_{C}\right)_{0} \backslash\left(Q_{B}\right)_{0}$ e nem de $\left(Q_{A}\right)_{0} \backslash\left(Q_{B}\right)_{0}$ para $\left(Q_{B}\right)_{0}$, ou seja, $e_{B} \operatorname{Re}_{C}^{\prime}=0$ e $e_{A}^{\prime} \operatorname{Re}_{B}=0$.

O seguinte esquema é útil para vizualização dessa definição, onde as flechas denotam o sentido possível para os caminhos em $Q_{R}$ :

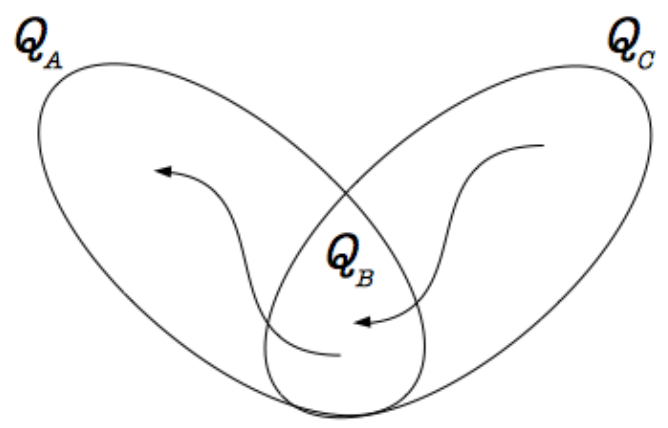

O produto fibrado do Exemplo 3.1.16 não é orientado pois $\delta$ é um caminho não nulo de $\left(Q_{A}\right)_{0} \backslash\left(Q_{B}\right)_{0}$ para $\left(Q_{B}\right)_{0}$. Vejamos alguns exemplos de produto fibrado orientado:

Exemplo 3.2.3 A álgebra hereditária de tipo infinito dada por

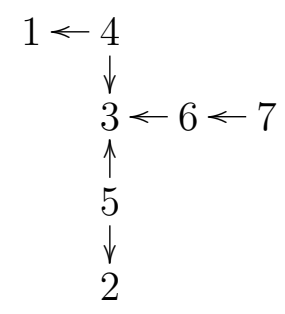

é um produto fibrado orientado das álgebras hereditárias de tipo finito:

\begin{tabular}{|c|c|c|c|}
\hline \multirow{6}{*}{$Q_{A}=$} & $1<4$ & 4 & 4 \\
\hline & & & $\downarrow$ \\
\hline & $Q_{B}=$ & $Q_{C}=$ & $\begin{array}{l}3<6<7 \\
\uparrow\end{array}$ \\
\hline & & & $\frac{1}{5}$ \\
\hline & & & $\downarrow$ \\
\hline & & & 2 \\
\hline
\end{tabular}


Exemplo 3.2.4 ([Lev04]) A álgebra cuja aljava ordinária com relações é

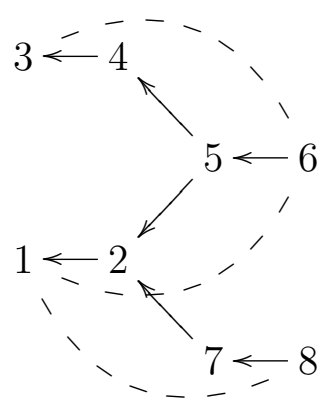

é um produto fibrado orientado de $A \rightarrow B$ e $C \rightarrow B$, onde $A, B$ e $C$ são as álgebras hereditárias dadas pelas aljavas:

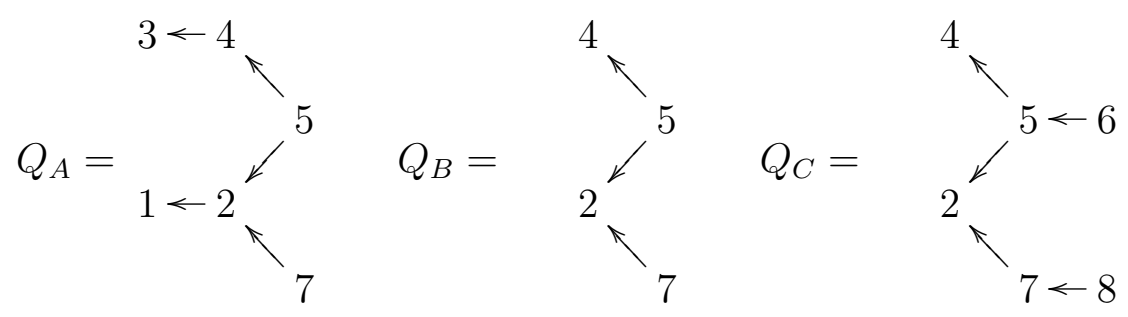

\subsubsection{Módulos sobre um produto fibrado orientado}

No último exemplo há $R$-módulos que não estão em ind $A \cup \operatorname{ind} C$ como, por exemplo, o $R$-módulo ${ }_{2}^{8}{ }_{2}^{5}{ }_{3}{ }_{3}$. Já no Exemplo 3.2.3 é fácil ver que existem infinitos desses módulos, isto é, infinitos módulos que não estão em ind $A \cup$ ind $C$. Veremos que tais $R$-módulos podem ser escritos como um produto fibrado entre $A$-módulos e $C$-módulos.

Proposição 3.2.5 Seja $R$ o produto fibrado orientado dos epimorfismos $A \rightarrow B$ e $C \rightarrow B$. Se $M$ é um R-módulo então existem epimorfismos $f: X_{A} \rightarrow Z_{B}$ e $g: Y_{C} \rightarrow Z_{B}$ tais que $M$ é o produto fibrado de $f$ e $g$.

Prova. Seja $M$ um $R$-módulo e consideremos $X_{A}=M / M e_{C}^{\prime} R, Y_{C}=M / M e_{A}^{\prime} R$ e $Z_{B}=$ $M / M\left(e_{A}^{\prime}+e_{C}^{\prime}\right) R$. Sejam $f: X_{A} \rightarrow Z_{B}$ e $g: Y_{C} \rightarrow Z_{B}$ os epimorfismos naturais. Pela Proposição 3.1.7 basta verificar que $M R e_{C}^{\prime} R \cap M R e_{A}^{\prime} R=0$. De fato, pelo Lema 3.2.1, temos $M R e_{C}^{\prime} R \cap M R e_{A}^{\prime} R=M R e_{C}^{\prime} R e_{B} \cap M R e_{A}^{\prime} R e_{B} \subseteq M R e_{A}^{\prime} R e_{B}=0$ pois pela definição de produto fibrado orientado temos $e_{A}^{\prime} R e_{B}=0$.

Corolário 3.2.6 Seja $R$ o produto fibrado orientado dos epimorfismos $A \rightarrow B$ e $C \rightarrow B$. Se $M$ é um R-módulo então $M$ é o produto fibrado de $M \otimes_{R} A \rightarrow M \otimes_{R} B$ e $M \otimes_{R} C \rightarrow M \otimes_{R} B$. 
Exemplo 3.2.7 Vejamos o que acontece no exemplo 3.2.4 com o módulo $M={ }_{7}^{8}{ }_{2}^{5}{ }_{3}$. Nesse caso, temos $e_{A}^{\prime}=e_{1}+e_{3}$ e $e_{C}^{\prime}=e_{6}+e_{8}$ e portanto $M R e_{C}^{\prime} R={ }_{2}^{8}$ e $M R e_{A}^{\prime} R=3$. Finalmente, $M_{A}={ }_{3}^{5}, M_{C}={ }_{7}^{8}{ }_{2}^{5}$ e $M_{B}={ }_{4}^{5}$.

Proposição 3.2.8 Seja $R$ o produto fibrado orientado dos epimorfismos $A \rightarrow B$ e $C \rightarrow B$. Então,

(a) $M e_{A}$ é um $R$-submódulo de $M$ e $M e_{B}$ é um $R$-submódulo de $M e_{C}$.

(b) $M e_{C}$ é um quociente de $M$ e $M e_{B}$ é um quociente de $M e_{A}$.

Prova. Para (a) basta ver que as inclusões naturais são monomorfismos de $R$-módulos. Como $M e_{A}$ é um $A$-módulo então, para $m \in M$, o produto por um elemento $r$ de $R$ é dado por $m e_{A} \cdot r=m e_{A} r e_{A}$ e como $Q_{A}$ é fechada para sucessores segue que $e_{A} r e_{A}=e_{A} r$ e portanto $m e_{A} r e_{A}=m e_{A} r$ que é o produto em $M$, isso mostra que $M e_{A}$ é submódulo de $M$. Analogamente, temos que $M e_{B}$ é submódulo de $M e_{C}$ pois $m e_{B} r e_{B}=m e_{B} e_{C} r e_{C}$.

Para (b) vejamos que o produto por $e_{C}$ de $M$ para $M e_{C}$ e o produto por $e_{B}$ de $M e_{A}$ para $M e_{B}$ são epimorfismos de $R$-módulos. Para o primeiro caso basta ver que $m r e_{C}=m e_{C} e_{C}$ para $m$ em $M$ e $r$ em $R$. De fato, como $Q_{C}$ é fechado para predecessores então vale $r e_{C}=e_{C} r e_{C}$ e portanto mre $_{C}=m_{C} r e_{C}$. Analogamente, temos que $e_{B} r e_{B}=e_{A} r e_{B}$ e portanto ambas as aplicações são morfismos de $R$-módulos que são obviamente sobrejetoras.

\subsubsection{Projetivos, Injetivos e Dimensão global}

Seja $R$ o produto fibrado de $A \rightarrow B$ e $C \rightarrow B$. Vimos nos Exemplos 3.2.3 e 3.2.4 que existem $R$-módulos indecomponíveis que não são nem $A$-módulos e nem $C$-módulos. Veremos, porém, que esse não é o caso para projetivos e injetivos quando o produto fibrado é orientado. Usaremos a seguinte notação: $P_{S}(i)$ denota o projetivo indecomponível associado ao idempotente $e_{i} \in S$ e $I_{S}(i)$ denota o injetivo indecomponível associado ao idempotente $e_{i} \in S$. Quando $S=R$ omitiremos o $R$ e escreveremos apenas $P(i)$ e $I(i)$.

Lema 3.2.9 Seja $R$ o produto fibrado orientado dos epimorfismos $A \rightarrow B$ e $C \rightarrow B$. Então,

1. $P(i) \cong P_{A}(i)$ para todo $i \in\left(Q_{A}\right)_{0}$;

2. $P(i) \cong P_{C}(i)$ para todo $i \in\left(Q_{C}\right)_{0} \backslash\left(Q_{B}\right)_{0}$;

3. $P_{C}(i) \cong P_{B}(i)$ para todo $i \in\left(Q_{B}\right)_{0}$;

4. $I(i) \cong I_{C}(i)$ para todo $i \in\left(Q_{C}\right)_{0}$;

5. $I(i) \cong I_{C}(i)$ para todo $i \in\left(Q_{A}\right)_{0} \backslash\left(Q_{B}\right)_{0}$; 
6. $I_{A}(i) \cong I_{B}(i)$ para todo $i \in\left(Q_{B}\right)_{0}$.

Prova. Para cada $i \in Q_{0}$ temos $P(i)=e_{i} R$. Se $i \in\left(Q_{A}\right)_{0}$, como o produto fibrado é orientado, temos $e_{i} R e_{C}^{\prime}=0$. Logo,

$$
e_{i} R=e_{i} e_{A} R e_{A} \cong e_{i} A=P_{A}(i) .
$$

Se $i \in\left(Q_{C}\right)_{0} \backslash\left(Q_{B}\right)_{0}$ então, como $e_{i} R e_{A}^{\prime}=0$, temos

$$
P(i)=e_{i} R=e_{i} e_{C} R e_{C} \cong e_{i} C=P_{C}(i) .
$$

Para $i \in\left(Q_{B}\right)_{0}$, como $e_{i} C e_{C}^{\prime}=0$, temos

$$
P_{C}(i)=e_{i} C=e_{i} e_{B} C e_{B} \cong e_{i} B=P_{B}(i) \text {. }
$$

Para os módulos injetivos a demonstração é simétrica.

Por simplicidade de notação, usaremos muitas vezes o sinal de igualdade no lugar de $\cong$, escrevendo, por exemplo que $P(i)=P_{A}(i)$ quando $i$ está em $\left(Q_{A}\right)_{0}$.

Segue deste último lema que o produto fibrado orientado preserva a dimensão projetiva dos $A$-módulos e a dimensão injetiva dos $C$-módulos, como veremos a seguir. Denotaremos para um $S$-módulo, $\operatorname{dp}_{S} M$ a dimensão projetiva de $M$ e $\operatorname{di}_{S} M$ a dimensão injetiva de $M$. Quando $S=R$ omitiremos o $R$ escrevendo apenas $\operatorname{dp} M$ e $\operatorname{di} M$.

Proposição 3.2.10 Seja $R$ o produto fibrado orientado dos epimorfismos $A \rightarrow B$ e $C \rightarrow B$. Então,

(a) para todo $M \in \operatorname{ind} A$ vale $\operatorname{dp} M=\operatorname{dp}_{A} M$

(b) para todo $M \in \operatorname{ind} C$ vale $\operatorname{di} M=\operatorname{di}_{C} M$

Prova. Segue do lema anterior que se $M$ é um $A$-módulo indecomponível então $M$ é projetivo como $A$-módulo se, e somente se, $M$ é projetivo como $R$-módulo.

Seja $M$ um $A$-módulo indecomponível e $0 \rightarrow K \rightarrow P_{n} \rightarrow \cdots \rightarrow P_{1} \rightarrow P_{0} \rightarrow M \rightarrow 0$ uma sequência exata minimal em $\bmod A$ com cada $P_{j}$ um $A$-módulo projetivo. Pelo lema anterior cada $P_{j}$ é um $R$-projetivo e portanto $\mathrm{dp}_{R} M \leq \mathrm{dp}_{A} M$. Por outro lado, seja $0 \rightarrow K \rightarrow P_{n} \rightarrow$ $\cdots \rightarrow P_{1} \rightarrow P_{0} \rightarrow M \rightarrow 0$ uma sequência exata minimal em $\bmod R \operatorname{com}$ cada $P_{j}$ um $R$ módulo projetivo. Como $M$ é um $A$-módulo então top $M$ é também um $A$-módulo e portanto $P_{0}$ é um $A$-módulo. Como $\operatorname{ker}\left(P_{0} \rightarrow M\right)$ também é um $A$-módulo pois é um submódulo de $P_{0}$ então por recorrência temos que cada $P_{i}$ é um $A$-módulo, portanto $A$-projetivo, e da mesma forma $K \in \bmod A$. Então tal sequência está em $\bmod A$ e portanto vale também a desigualdade $\operatorname{dp}_{A} M \leq \operatorname{dp}_{R} M$ mostrando que vale a igualdade.

A demonstração de (b) é dual. 
Denotemos a partir de agora por $C^{\prime}$ a soma direta dos projetivos $P(i)$ com $i \in\left(Q_{C}\right)_{0} \backslash$ $\left(Q_{B}\right)_{0}$ e por $D A^{\prime}$ a soma direta dos injetivos $I(i)$ com $i \in\left(Q_{A}\right)_{0} \backslash\left(Q_{B}\right)_{0}$.

Observação 3.2.11 Podemos escrever o $R$-módulo $C$ da seguinte forma

$$
\begin{aligned}
C & =\bigoplus\left\{P_{C}(i) \mid i \in\left(Q_{C}\right)_{0}\right\} \\
& =\bigoplus\left\{P_{C}(i) \mid i \in\left(Q_{B}\right)_{0}\right\} \oplus \bigoplus\left\{P_{C}(i) \mid i \in\left(Q_{C}\right)_{0} \backslash\left(Q_{B}\right)_{0}\right\} \\
& \cong \bigoplus\left\{P_{B}(i) \mid i \in\left(Q_{B}\right)_{0}\right\} \oplus \bigoplus\left\{P(i) \mid i \in\left(Q_{C}\right)_{0} \backslash\left(Q_{B}\right)_{0}\right\} \\
& =B \oplus C^{\prime}
\end{aligned}
$$

De forma análoga podemos escrever $D A=D A^{\prime} \oplus D B, R=A \oplus C^{\prime}$ e $D R=D A^{\prime} \oplus D C$.

Para a dimensão global do produto fibrado, denotada por dim.gl $R$, usaremos o seguinte resultado de Wiseman ([Wis85] (3.1)), que aqui será escrito no nosso contexto, ou seja, para álgebras de dimensão finita sobre o corpo $k$.

Lema 3.2.12 Considere o diagrama

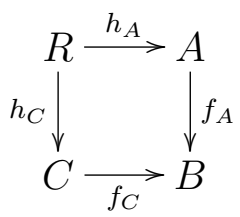

com $h_{A}$ um epimorfismo e $R$ o produto fibrado de $f_{A}$ e $f_{C}$. Suponha que $\max \{\operatorname{dp} A, \operatorname{dp} C\} \leq n$. Então

$$
\operatorname{dim} . \mathrm{gl} R \leq \max \{\operatorname{dim} . \mathrm{gl} A, \operatorname{dim} \cdot \mathrm{gl} C\}+n .
$$

Para o produto fibrado orientado, o Lema 3.2.9 e a Proposição 3.2.10 nos darão uma versão mais simplificada desse resultado.

Proposição 3.2.13 Seja $R$ o produto fibrado orientado dos epimorfismos $A \rightarrow B$ e $C \rightarrow B$. Então

$$
\operatorname{dim} . g l R \leq \max \{\operatorname{dim} . g l A, \operatorname{dim} . g l C\}+\operatorname{dp}_{A} B .
$$

Prova. Pelo Lema 3.2.9 temos que $A$ é um $R$-módulo projetivo e portanto $\operatorname{dp} A=0$. Também temos pela última observação que $C=B \oplus C^{\prime}$. Visto que $C^{\prime}$ é um $R$-módulo projetivo então $\operatorname{dp} C=\operatorname{dp} B$. Basta agora aplicar o último lema e notar que pela Proposição 3.2.10 temos $\mathrm{dp} B=\mathrm{dp}_{A} B$ pois $B$ é um $A$-módulo.

Como caso particular, se tivermos $A$ uma álgebra hereditária então $\operatorname{dp} B \leq 1$ e portanto a dimensão global do produto fibrado $R$ só depende da álgebra $C$. E no caso de ambas $A$ e $C$ serem hereditárias temos que a dimensão global de $R$ não ultrapassa 2. 
Corolário 3.2.14 Seja $R$ o produto fibrado orientado dos epimorfismos $A \rightarrow B$ e $C \rightarrow B$. Se A é uma álgebra hereditária então $\operatorname{dim} . g l R \leq \operatorname{dim} . g l C+1$. Em particular, se $C$ também é hereditária então dim.gl $R \leq 2$.

Com isso chegamos a seguinte generalização de [Lev04] (5.2.4).

Corolário 3.2.15 Seja $R$ o produto fibrado orientado dos epimorfismos $A \rightarrow B$ e $C \rightarrow B$ onde $A$ e $C$ são álgebras hereditárias. Então, $R$ é uma álgebra shod se, e somente se, $R$ é quase inclinada.

Prova. Da seção 1.4, temos que uma álgebra é quase inclinada se, e somente se, ela é shod e tem dimensão global no máximo 2. O resultado segue, portanto, do corolário anterior.

\subsection{Casos particulares do produto fibrado orientado}

Nesta seção consideramos algumas hipóteses adicionais ao produto fibrado orientado que nos permitirá concluir que todos os $R$-módulos são $A$-módulos ou $C$-módulos. O primeiro, produto fibrado com condições $*$, foi definido em [IPTZ87] e o resultado citado é devido a este trabalho. O segundo, produto fibrado Nakayama orientado, foi inspirado no primeiro e definido em [Lev04] onde mostra-se ainda que a aljava de Auslander-Reiten de $R$ é exatamente a soma amalgamada das aljavas de Auslander-Reiten de $A$ e de $C$. Finalmente, definiremos o produto fibrado Dynkin orientado que generaliza os dois primeiros. Mostraremos que para esse caso ainda vale que $\operatorname{ind} R=\operatorname{ind} A \cup \operatorname{ind} C$.

\section{Produto fibrado com "condições *"}

Definição 3.3.1 ([IPTZ87]) Seja $R$ o produto fibrado de $A \rightarrow B$ e $C \rightarrow B$. Dizemos que $R$ satisfaz as condições $*$ se:

1. não há caminho não nulo de $\left(Q_{B}\right)_{0}$ para $\left(Q_{C}\right)_{0} \backslash\left(Q_{B}\right)_{0}$ e nem de $\left(Q_{A}\right)_{0} \backslash\left(Q_{B}\right)_{0}$ para $\left(Q_{B}\right)_{0} ;$

2. $Q_{B}$ é da forma $a_{t} \leftarrow a_{t-1} \leftarrow \cdots \leftarrow a_{1}$ com $t \geq 1$, sem relações;

3. dados uma flecha $\alpha: x \rightarrow$ y em $\left(Q_{B}\right)_{1}$ e um caminho $\varphi: z \rightsquigarrow$ y com $z$ em $\left(Q_{C}\right)_{0} \backslash\left(Q_{B}\right)_{0}$ existe um caminho $\psi: z \rightsquigarrow x$ tal que $\psi \alpha-\varphi$ está em $I_{C}$.

O primeiro item nos diz justamente que se $R$ satisfaz as condições $*$ então ele é um produto fibrado orientado.

Teorema 3.3.1 ([IPTZ87](3.5)) Seja $R$ o produto fibrado de $A \rightarrow B$ e $C \rightarrow B$ satisfazendo as condições $*$. Então, $\operatorname{ind} R=\operatorname{ind} A \cup \operatorname{ind} C e \operatorname{ind} B=\operatorname{ind} A \cap \operatorname{ind} C$.

Veremos mais adiante que esse resultado pode ser estendido para outros casos de produtos fibrados orientados. 


\section{Produto fibrado Nakayama orientado}

Para esse tipo de produto fibrado J. Levesque em [Lev04] (ou [Lev08] ) mostrou que se $A$ e $C$ são álgebras hereditárias então $R$ é uma álgebra inclinada.

Para tanto ela construiu, a partir de módulos inclinantes de $A, B$ e $C$, um módulo inclinante $T$ tal que End $T$ é uma álgebra hereditária.

Chegaremos também a esse resultado com outras técnicas que serão discutidas no Capítulo 4 .

Definição 3.3 .2 ([Lev04]) Seja $R$ o produto fibrado de $A \rightarrow B$ e $C \rightarrow B$. Dizemos que $R$ é o produto fibrado Nakayama orientado se:

i) Não há caminhos de $\left(Q_{B}\right)_{0}$ para $\left(Q_{C}\right)_{0} \backslash\left(Q_{B}\right)_{0}$ e nem de $\left(Q_{A}\right)_{0} \backslash\left(Q_{B}\right)_{0}$ para $\left(Q_{B}\right)_{0}$.

ii) B é uma álgebra hereditária Nakayama e as componentes conexas $Q_{B_{1}}, Q_{B_{2}}, \ldots, Q_{B_{r}}$ de $Q_{B}$ são da forma $Q_{B_{i}}=a_{i, t_{i}} \leftarrow a_{i, t_{i-1}} \leftarrow \cdots \leftarrow a_{i, 1}$ com $1 \leq i \leq r$ e $t_{i} \geq 1$.

iii) $E m Q_{B}$ somente fontes são finais de flechas de $\left(Q_{C}\right)_{1} \backslash\left(Q_{B}\right)_{1}$ e somente poços são inicios de flechas de $\left(Q_{A}\right)_{1} \backslash\left(Q_{B}\right)_{1}$.

iv) Nenhuma relação minimal de $R$ tem origem em $\left(Q_{B}\right)_{0}$.

Notemos que o primeiro item dessa definição é o item (1) das condições $*$, ou seja, que tal produto fibrado é orientado. No item (ii) vemos que cada componente conexa de $Q_{B}$ é da forma de (2) das condições $*$. Veremos adiante que o item (3) das condições $*$ é também válido.

\subsubsection{Produto fibrado Dynkin orientado}

Inspirados na demonstração do Teorema 3.3.1 de que não há módulos indecomponíveis que não sejam nem $A$-módulos e nem $C$-módulos, chegamos em outros casos em que esse resultado também é válido e definimos o produto fibrado Dynkin orientado.

Definição 3.3.3 Seja $R$ um produto fibrado orientado de $A \rightarrow B$ e $C \rightarrow B$. Dizemos que $R$ é um produto fibrado Dynkin orientado se:

(a) Cada componente conexa de $Q_{B}$ é de tipo Dynkin com um único poço, sem relações.

(b) Dados uma flecha $\alpha: x \rightarrow$ y em $\left(Q_{B}\right)_{1}$ e um caminho $\varphi: z \rightsquigarrow$ y com z em $\left(Q_{C}\right)_{0} \backslash\left(Q_{B}\right)_{0}$ existe um caminho $\psi: z \rightsquigarrow x$ tal que $\psi \alpha-\varphi$ está em $I_{C}$.

Trivialmente o produto fibrado com condições $*$ é um produto fibrado Dynkin orientado. Veremos que o produto fibrado Nakayama orientado também é. Para isso, falta verificar que a condição (b) da última definição é satisfeita. Isso decorre do seguinte lema:

Lema 3.3.2 Se $R$ é o produto fibrado Nakayama orientado de $A \rightarrow B$ e $C \rightarrow B$ então, dados uma flecha $\alpha: x \rightarrow y$ em $\left(Q_{B}\right)_{1}$ e um caminho $\varphi: z \rightsquigarrow y \operatorname{com} z$ em $\left(Q_{C}\right)_{0} \backslash\left(Q_{B}\right)_{0}$ existe um caminho $\psi: z \rightsquigarrow x$ tal que $\psi \alpha=\varphi$. 
Prova. Sejam $\alpha: x \rightarrow y$ uma flecha em $\left(Q_{B}\right)_{1}$ e $\varphi: z \rightsquigarrow y$ um caminho com $z$ em $\left(Q_{C}\right)_{0} \backslash\left(Q_{B}\right)_{0}$. Escreva $\varphi=\psi \beta$ onde $\beta: z_{0} \rightarrow y$ é uma flecha em $\left(Q_{C}\right)_{1}$ e $\psi: z \rightsquigarrow z_{0}$ é um caminho. Como y não é uma fonte em $Q_{B}$ então pelo item (ii) da Definição 3.3.2 temos que $z_{0}$ está também em $\left(Q_{B}\right)_{0}$. Além disso pela forma da aljava $Q_{B}$ segue que $z_{0}=x$ e ainda que $\alpha=\beta$. Portanto, temos que $\varphi=\psi \alpha$.

Proposição 3.3.3 Seja $R$ o produto fibrado Dynkin orientado de $A \rightarrow B$ e $C \rightarrow B$. Se $C$ é uma álgebra hereditária então cada componente conexa de $Q_{B}$ é de tipo $\mathbb{A}_{n}$ linearmente orientada.

Prova. Fixemos $Q$ uma componente conexa de $Q_{B}$ e suponhamos que $Q$ não é de tipo $\mathbb{A}_{n}$ linearmente orientada. Da lista de Dynkin orientado com um único poço (seção 2.1) vemos que $Q$ possui um dos seguintes 4 formatos, onde "1" denota o único poço e "•" são fontes:

Caso 1 Na lista 2.1 a aljava: $\mathbb{A}_{n} \cdot 2$

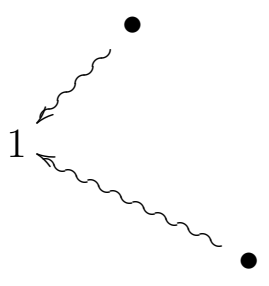

Caso 2 Na lista 2.1 as aljavas: $\mathbb{D}_{n} .2, \mathbb{E}_{6} .4, \mathbb{E}_{7} .4$ e $\mathbb{E}_{8} .4$.

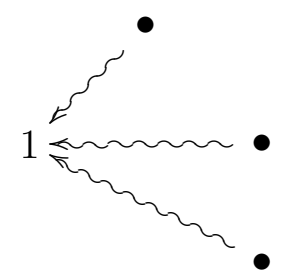

Caso $3 \mathrm{Na}$ lista 2.1 as aljavas: $\mathbb{D}_{n} .3, \mathbb{E}_{6} .2, \mathbb{E}_{7} .2, \mathbb{E}_{7} .5, \mathbb{E}_{7} \cdot 6, \mathbb{E}_{8} \cdot 2, \mathbb{E}_{8} .5, \mathbb{E}_{8} .6$ e $\mathbb{E}_{8} .7$.

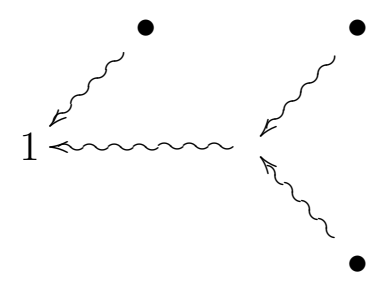

Caso $4 \mathrm{Na}$ lista 2.1 as aljavas: $\mathbb{D}_{n} .1, \mathbb{D}_{n} .4, \mathbb{E}_{6} .1, \mathbb{E}_{6} .3, \mathbb{E}_{7} .1, \mathbb{E}_{7} .3, \mathbb{E}_{7} .7, \mathbb{E}_{8} .1, \mathbb{E}_{8} .3$ e $\mathbb{E}_{8} .8$.

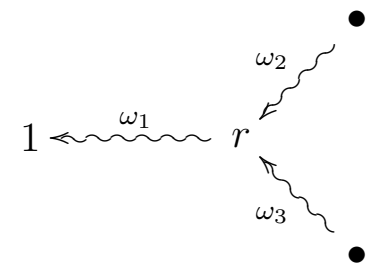


Como $Q_{C}$ é conexa então existe um caminho de $i \in\left(Q_{C}\right)_{0} \backslash\left(Q_{B}\right)_{0}$ para $j \in Q_{0}$.

Nos casos 1, 2 e 3 considerers o caminho $\phi: i \rightsquigarrow j \rightsquigarrow 1$. Em cada um desses casos existe uma flecha $\alpha: x \rightarrow 1$ que não está em $\phi$. Por (b) da Definição 3.3.3 existe um caminho $\psi: i \rightsquigarrow x$ tal que $\psi \alpha-\phi \in I_{C}$. Uma contradição pois $C$ é hereditária.

Para o caso 4 , se $j$ está em $\omega_{2}$ ou em $\omega_{3}$ podemos repetir o argumento anterior trocando o vértice "1" por "r". Suponhamos então, que $j$ está em $\omega_{1}$ que pode ser escrito como: $\omega_{1}: 1 \longleftarrow 2 \longleftarrow \cdots \ll j \leftarrow^{\alpha_{j}} j+1 \stackrel{\alpha_{j+1}}{\longleftarrow} \cdots \stackrel{\alpha_{r-1}}{\longleftarrow} r$. Seja $\varphi$ o caminho de $i$ para $j$. Por (b) da Definição 3.3 .3 e como $I_{C}=0$ então $\varphi$ é da forma $\varphi=\varphi_{0} \alpha_{r-1} \cdots \alpha_{j+1} \alpha_{j}$ onde $\varphi_{0}: i \rightsquigarrow r$. Mas há duas flechas que chegam em $r$ e novamente chegamos em uma relação em $I_{C}$ o que é uma contradição.

Portanto, a componente conexa $Q$ é de tipo $\mathbb{A}_{n}$ linearmente orientada.

Exemplo 3.3.4 Considere as álgebras hereditárias $A, B$ e $C$ dadas, respectivamente, pelas seguintes aljavas: $2 \leftarrow 3 \leftarrow 4 \leftarrow 5 \quad, 2<3<4<5 \quad, 2<3<4<5$

Neste caso o produto fibrado $R$ é dado pela aljava abaixo com relações $\gamma_{i} \beta_{3} \beta_{2} \beta_{1} \alpha_{1}=$ $\gamma_{i} \beta_{3} \beta_{2} \alpha_{2}=0$.

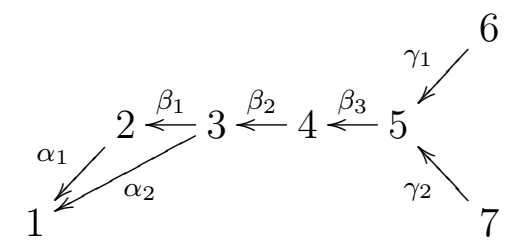

Note que se trata de um produto fibrado Dynkin orientado, mas que não é Nakayama orientado mesmo $Q_{B}$ sendo de tipo $\mathbb{A}_{4}$ linearmente orientado, pois não satisfaz o o item (iii) da Definição 3.3.2.

Vejamos agora um exemplo de produto fibrado Dynkin orientado onde $Q_{B}$ não é de tipo $\mathbb{A}_{n}$.

Exemplo 3.3.5 A álgebra dada pela seguinte aljava com relações

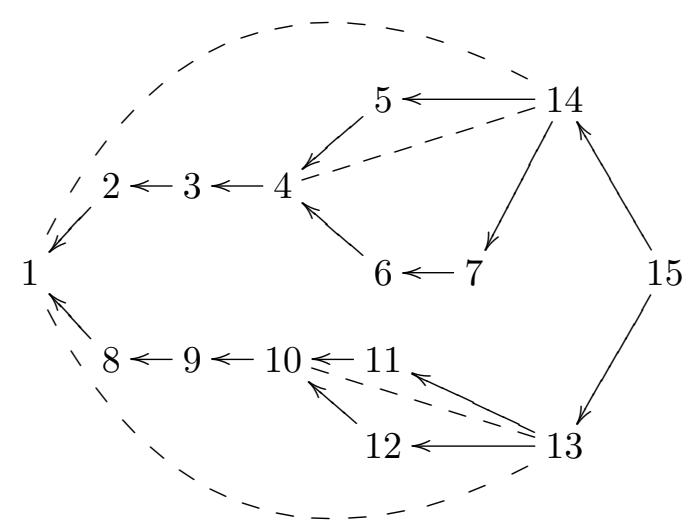


é um produto fibrado Dynkin orientado onde $\left(Q_{A}\right)_{0} \backslash\left(Q_{B}\right)_{0}=\{1\}$ e $\left(Q_{C}\right)_{0} \backslash\left(Q_{B}\right)_{0}=$ $\{13,14,15\}$. Neste caso, $Q_{B}$ tem duas componentes conexas, uma de tipo $\mathbb{D}_{5}$ e uma de tipo $\mathbb{E}_{6}$.

Com a mesma prova dada em [IPTZ87] é possível mostrar que o resultado do Teorema 3.3.1 também é válido para o produto fibrado Dynkin orientado. Reproduziremos tal demonstração para esse caso, mas para isso precisaremos do Teorema 2.2.2, do segundo capítulo.

Teorema 3.3.6 Se $R$ é o produto fibrado Dynkin orientado de $A \rightarrow B$ e $C \rightarrow B$ então $\operatorname{ind} R=\operatorname{ind} A \cup \operatorname{ind} C e \operatorname{ind} B=\operatorname{ind} A \cap \operatorname{ind} C$.

Prova. Já vimos que ind $A$ e ind $C$ são subcategorias de ind $R$ e também pelos suportes das representações é fácil ver que $\operatorname{ind} B=\operatorname{ind} A \cap \operatorname{ind} C$. Faremos a outra desigualdade também utilizando representações de módulos.

Seja $M=\left(\left(V_{x}\right)_{x \in\left(Q_{R}\right)_{0}},\left(f_{\alpha}\right)_{\alpha \in\left(Q_{R}\right)_{1}}\right)$ uma $\left(Q_{R}, I_{R}\right)$-representação de um $R$-módulo indecomponível. Definiremos uma $\left(Q_{R}, I_{R}\right)$-representação $\operatorname{Im} M=\left(\left(W_{x}\right)_{x \in\left(Q_{R}\right)_{0}},\left(g_{\alpha}\right)_{\alpha \in\left(Q_{R}\right)_{1}}\right)$ da seguinte forma:

- para cada vértice $x$ em $\left(Q_{R}\right)_{0}$ considere $W_{x}$ a soma das imagens das aplicações $f_{\omega}: V_{y} \rightarrow$ $V_{x}$, onde $\omega$ é um caminho de $y$ para $x$ com $y$ em $\left(Q_{C}\right)_{0} \backslash\left(Q_{B}\right)_{0}$;

- para cada $\alpha: x \rightarrow y$ em $\left(Q_{R}\right)_{1}$, considere $g_{\alpha}=\left.f_{\alpha}\right|_{W_{x}}$ a restrição de $f_{\alpha}$ a $W_{x}$.

Observe que se $x$ está em $\left(Q_{C}\right)_{0} \backslash\left(Q_{B}\right)_{0}$ então $W_{x}=V_{x}$ pois para o caminho trivial $\epsilon_{x}$ temos $\operatorname{im} f_{\epsilon_{x}}=V_{x}$. Por outro lado, se $x$ está em $\left(Q_{A}\right)_{0} \backslash\left(Q_{B}\right)_{0}$ então $W_{x}=0$ pois, pela orientação do produto fibrado, não existe caminho não nulo de $\left(Q_{C}\right)_{0} \backslash\left(Q_{B}\right)_{0}$ para $\left(Q_{A}\right)_{0} \backslash$ $\left(Q_{B}\right)_{0}$. Portanto, $\operatorname{Im} M$ é uma $\left(Q_{C}, I_{C}\right)$-representação, enquanto $M / \operatorname{Im} M$ é uma $\left(Q_{A}, I_{A}\right)$ representação. Mostrarenos que $\operatorname{Im} M=0$ ou $\operatorname{Im} M=M$ de onde seguirá o resultado.

Afirmação: Para cada flecha $\alpha: x \rightarrow y$ em $\left(Q_{B}\right)_{1}$, a aplicação $g_{\alpha}: W_{x} \rightarrow W_{y}$ é sobrejetora.

Seja $b$ em $\operatorname{im} f_{\omega}$ para algum caminho $\omega$ de $z \in\left(Q_{C}\right)_{0} \backslash\left(Q_{B}\right)_{0}$ para $y$ e seja $a$ em $V_{z}$ tal que $f_{\omega}(a)=b$. Pelo item 3 da Definição 3.3.1, existe um caminho $\psi$ de $z$ em $x$ tal que $\psi \alpha-\omega$ está em $I_{R}$. Então, $f_{\alpha} \circ f_{\psi}=f_{\omega}$ e portanto $b=f_{\omega}(a)=f_{\alpha}\left(f_{\psi}(a)\right)=g_{\alpha}\left(f_{\psi}(a)\right)$ pois $f_{\psi}(a)$ está em $\operatorname{im} f_{\psi} \subseteq W_{x}$ e $\left.f_{\alpha}\right|_{W_{x}}=g_{\alpha}$. Logo, $g_{\alpha}$ é sobrejetora como queríamos.

Segue dessa afirmação e do Teorema 2.2.2 que $\left.(\operatorname{Im} M)\right|_{Q_{B}}$ é uma $Q_{B}$-representação de um módulo injetivo e portanto a seguinte sequência exata

$$
0 \rightarrow \operatorname{Im} M \rightarrow M \rightarrow M / \operatorname{Im} M \rightarrow 0
$$

restrita a $Q_{B}$ cinde. Vejamos que ela também cinde em $\left(Q_{R}, I_{R}\right)$.

Seja $\iota: \operatorname{Im} M \rightarrow M$ a inclusão. Como a sequência cinde em $Q_{B}$, existe $h:\left.M\right|_{Q_{B}} \rightarrow$ $\left.(\operatorname{Im} M)\right|_{Q_{B}}$ tal que $h \circ \iota^{\prime}=$ id onde $\iota^{\prime}$ é a restrição de $\iota$ a $\left.(\operatorname{Im} M)\right|_{Q_{B}}$. Considere a seguinte 
extensão $l$ de $h$, dada, para cada $x$ de $\left(Q_{R}\right)_{0}$, por:

$$
l_{x}=\left\{\begin{array}{ccc}
h_{x} & \text { se } & x \in\left(Q_{B}\right)_{0} \\
\operatorname{id}_{V_{x}} & \text { se } & x \in\left(Q_{C}\right)_{0} \backslash\left(Q_{B}\right)_{0} \\
0 & \text { se } & x \in\left(Q_{A}\right)_{0} \backslash\left(Q_{B}\right)_{0}
\end{array}\right.
$$

Como o produto fibrado é orientado, é fácil mostrar que $l$ é um morfismo de $\left(Q_{R}, I_{R}\right)$ representações e que $l \circ \iota=i d_{\operatorname{Im} M}$. Portanto a sequência cinde e segue que $M \cong \operatorname{Im} M \oplus$ $M / \operatorname{Im} M$. Mas, como $M$ é indecomponível segue que $\operatorname{Im} M=0$ ou $\operatorname{Im} M=M$ e portanto $M$ é uma $\left(Q_{A}, I_{A}\right)$-representação ou uma $\left(Q_{C}, I_{C}\right)$-representação, concluindo o resultado.

Uma consequência imediata é dada pelo seguinte corolário:

Corolário 3.3.7 Se $R$ é o produto fibrado Dynkin orientado de $A \rightarrow B$ e $C \rightarrow B$ então $R$ é de tipo finito se, e somente se, $A$ e $C$ são de tipo finito.

Corolário 3.3.8 Se $R$ é o produto fibrado Dynkin orientado de $A \rightarrow B$ e $C \rightarrow B$ com $A$ e $B$ álgebras hereditárias então $R$ é quase inclinada.

Prova. Do Corolário 3.2.15, basta ver que $R$ é uma álgebra shod. Do Teorema 3.3.6 te$\operatorname{mos}$ que ind $R=\operatorname{ind} A \cup \operatorname{ind} C$. Então, se $M \in \operatorname{ind} A$ temos, pela Proposição 3.2.10, que $\operatorname{dp} M=\operatorname{dp}_{A} M$ e como $A$ é hereditária segue que $\operatorname{dp} M \leq 1$. Analogamente, se $M \in \operatorname{ind} C$ então, $\operatorname{di} M=\operatorname{di}_{C} M \leq 1$ pois $C$ é hereditária. Portanto, $R$ é uma álgebra shod. 


\section{Capítulo 4}

\section{Produto fibrado orientado e subcategorias de ind $R$}

Considere $R$ o produto fibrado orientado dos epimorfismos $A \rightarrow B$ e $C \rightarrow B$ como definido na Seção 3.2. Nesse capítulo iremos destacar algumas subcategorias que são fechadas para predecessores ou para sucessores da categoria de módulos do produto fibrado $R$. Com o auxílio dessas subcategorias mostraremos, sob certas condições, que um produto fibrado orientado $R$ é uma álgebra inclinada quando $A$ e $C$ são álgebras hereditárias. O que nos dará uma nova demonstração para [Lev08] (2.4.7) (ver Corolário 4.4.5).

Também analisamos situações semelhantes envolvendo outras classes de álgebras, como as colagens à direita e à esquerda, por exemplo. Para isso, na Seção 4.4, estudamos a relação entre as partes direita e esquerda das categorias de módulos das álgebras envolvidas.

Além disso, na Seção 4.3, conseguimos relacionar as aljavas de Auslander-Reiten de $R$ com as aljavas de Auslander-Reiten de $A, B$ e $C$.

Antes veremos, na primeira seção, que toda álgebra do tipo ada pode ser vista como um produto fibrado orientado.

Em todo o capítulo usaremos a noção de trisecção como na definição a seguir.

Definição 4.0.4 ([AAC ${ }^{+}$11]) Uma trisecção de ind $\Lambda$ é uma tripla de subcategorias plenas disjuntas $(\mathcal{A}, \mathcal{B}, \mathcal{C})$ tal que ind $\Lambda=\mathcal{A} \cup \mathcal{B} \cup \mathcal{C}$ e $\operatorname{Hom}(\mathcal{C}, \mathcal{B})=\operatorname{Hom}(\mathcal{C}, \mathcal{A})=\operatorname{Hom}(\mathcal{B}, \mathcal{A})=0$.

Se $(\mathcal{A}, \mathcal{B}, \mathcal{C})$ é uma trisecção de ind $\Lambda$ então a subcategoria $\mathcal{A}$ é fechada para predecessores e a subcategoria $\mathcal{C}$ é fechada para sucessores. Também, $\mathcal{B}$ é convexa em ind $\Lambda$, ou seja, se $M=M_{1} \rightarrow M_{2} \rightarrow \cdots \rightarrow M_{t}=N$ é um caminho em ind $\Lambda$ com $M$ e $N$ em $\mathcal{B}$ então cada $M_{i}$ para $i=1, \ldots, t$ também está em $\mathcal{B}$.

\subsection{Produto fibrado das álgebras suporte}

Seja $\mathcal{A}$ uma subcategoria plena de ind $\Lambda$ fechada para predecessores. Definimos sua álgebra suporte ${ }_{\mathcal{A}} \Lambda$ como a álgebra de endomorfismo da soma direta dos $\Lambda$-módulos projetivos indecomponíveis que estão em $\mathcal{A}$, ou seja, ${ }_{\mathcal{A}} \Lambda=$ End $\bigoplus\{P(x) \mid P(x) \in \mathcal{A}\}$. Dualmente, se $\mathcal{C}$ é uma subcategoria plena de ind $\Lambda$ fechada para sucessores, definimos a álgebra suporte $\Lambda_{\mathcal{C}}$ de $\mathcal{C}$ como $\Lambda_{\mathcal{C}}=$ End $\bigoplus\{I(x) \mid I(x) \in \mathcal{C}\}$. Temos as seguintes propriedades para as álgebras suporte. 
Lema 4.1.1 ([ACT09] (4.1)) Sejam $\mathcal{A}$ uma subcategoria plena de ind $\Lambda$ fechada para predecessores e $\mathcal{C}$ uma subcategoria plena de ind $\Lambda$ fechada para sucessores.

(a) Os $\Lambda$-módulos que estão em $\mathcal{A}$ têm uma estrutura natural de ${ }_{\mathcal{A}} \Lambda$-módulos indecomponiveis.

(b) $O s{ }_{\mathcal{A}} \Lambda$-módulos projetivos indecomponíveis são justamente os $\Lambda$-módulos projetivos que estão em $\mathcal{A}$.

(c) Para cada ${ }_{\mathcal{A}} \Lambda$-módulo $M$ temos que $\operatorname{dp}_{\left({ }_{\mathcal{A}} \Lambda\right)} M=\operatorname{dp}_{\Lambda} M$ e $\operatorname{di}_{\left({ }_{\mathcal{A}} \Lambda\right)} M \leq \operatorname{di}_{\Lambda} M$.

(a') Os $\Lambda$-módulos que estão em $\mathcal{C}$ têm uma estrutura natural de $\Lambda_{\mathcal{C}}$-módulos indecomponíveis.

(b') Os $\Lambda_{\mathcal{C}}$-módulos injetivos indecomponíveis são justamente os $\Lambda$-módulos injetivos que estão em $\mathcal{C}$.

(c') Para cada $\Lambda_{\mathcal{C}}$-módulo $M$ temos que $\operatorname{di}_{\left(\Lambda_{\mathcal{C})}\right.} M=\operatorname{di}_{\Lambda} M$ e $\operatorname{dp}_{\left(\Lambda_{\mathcal{C}}\right)} M \leq \operatorname{dp}_{\Lambda} M$.

Notemos que $\mathcal{A}$ é uma subcategoria de ind ${ }_{\mathcal{A}} \Lambda$ fechada para predecessores e que contém todos os ${ }_{\mathcal{A}} \Lambda$-módulos projetivos indecomponíveis. Dualmente, $\mathcal{C}$ é uma subcategoria de ind $\Lambda_{\mathcal{C}}$ fechada para sucessores que contém todos os $\Lambda_{\mathcal{C}}$-módulos injetivos indecomponíveis.

Observação 4.1.2 Se $\Lambda=k Q_{\Lambda} / I_{\Lambda}$ então a álgebra $A={ }_{\mathcal{A}} \Lambda$ pode ser escrita como $k Q_{A} / I_{A}$ onde $Q_{A}$ é a subaljava plena de $Q_{\Lambda}$ tal que o conjunto de vértices é $\left(Q_{A}\right)_{0}=\left\{x \in\left(Q_{\Lambda}\right)_{0} \mid\right.$ $P(x) \in \mathcal{A}\}$ e o ideal $I_{A}$ é $I_{\Lambda} \cap k Q_{A}$. Também $C=\Lambda_{\mathcal{C}}$ pode ser escrita como $k Q_{C} / I_{C}$ onde $Q_{C}$ é a subaljava plena de $Q_{\Lambda}$ tal que o conjunto de vertices é $\left(Q_{C}\right)_{0}=\left\{x \in\left(Q_{\Lambda}\right)_{0} \mid I(x) \in \mathcal{C}\right\}$ e o ideal $I_{C}$ é $I_{\Lambda} \cap k Q_{C}$.

Vejamos um exemplo de uma álgebra ada que pode ser escrita como produto fibrado das álgebras suporte das partes direita e esquerda.

Exemplo 4.1.3 Considere a álgebra $\Lambda$ com $\operatorname{rad}^{2} \Lambda=0$ (ou seja, qualquer caminho de comprimento maior ou igual a 2 é nulo) cuja aljava ordinária é

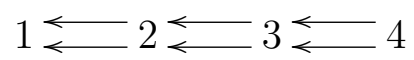

Neste caso, a parte esquerda de $\bmod \Lambda$ é dada pelos predecessores de $P_{3}$, ou seja, $\mathcal{L}_{\Lambda}=$ $\operatorname{Pred} P(3)$ e a parte direita pelos sucessores de $I(2)$, ou seja, $\mathcal{R}_{\Lambda}=\operatorname{Succ} I(2)$. Temos que $P(1), P(2), P(3)$ e I(1) estão em $\mathcal{L}_{\Lambda}$, enquanto $P(4), I(2), I(3)$ e I(4) estão em $\mathcal{R}_{\Lambda}$. Portanto, $\Lambda$ é uma álgebra ada.

Nesse caso, a álgebra suporte de $\mathcal{L}_{\Lambda}$ é $1 \longleftarrow 2 \longleftarrow 3$ com $\operatorname{rad}^{2}\left({ }_{\left(\mathcal{L}_{\Lambda}\right)} \Lambda\right)=0$ e a álgebra suporte de $\mathcal{R}_{\Lambda}$ é $2 \longleftarrow 3 \longleftarrow 4$ com $\operatorname{rad}^{2}\left(\Lambda_{\left(\mathcal{R}_{\Lambda}\right)}\right)=0$. Considere $B$ a álgebra com aljava $2 \longleftarrow 3$. Então, $\Lambda$ é o produto fibrado orientado dos epimorfismos naturais ${ }_{\left(\mathcal{L}_{\Lambda}\right)} \Lambda \rightarrow B$ e $\Lambda_{\left(\mathcal{R}_{\Lambda}\right)} \rightarrow B$. 
Veremos com o próximo resultado que tal construção sempre é possível para uma álgebra ada.

Teorema 4.1.4 Sejam $\Lambda=k Q_{\Lambda} / I_{\Lambda}$ uma álgebra, $\mathcal{A}$ uma subcategoria de ind $\Lambda$ fechada para predecessores e $\mathcal{C}$ fechada para sucessores tais que $\Lambda$ está em $\operatorname{add}(\mathcal{A} \cup \mathcal{C})$ ou $D \Lambda$ está em $\operatorname{add}(\mathcal{A} \cup \mathcal{C})$. Então, existe uma álgebra $B$ tal que $\Lambda$ é o produto fibrado orientado de ${ }_{\mathcal{A}} \Lambda \rightarrow B$ $e \Lambda_{\mathcal{C}} \rightarrow B$.

Prova. Faremos a prova supondo que $\Lambda$ está $\operatorname{em~} \operatorname{add}(\mathcal{A} \cup \mathcal{C})$. $\mathrm{O}$ caso $D \Lambda \in \operatorname{add}(\mathcal{A} \cup \mathcal{C})$ se mostra de forma análoga.

Denotemos ${ }_{\mathcal{A}} \Lambda$ por $A$ e $\Lambda_{\mathcal{C}}$ por $C$. Lembremos que, da Observação 4.1.2, podemos escrever $A \cong k Q_{A} / I_{A}$ e $C \cong k Q_{C} / I_{C}$ onde $Q_{A}$ e $Q_{C}$ são subaljavas plenas de $Q_{\Lambda} \operatorname{com}\left(Q_{A}\right)_{0}=\{x \in$ $\left.\left(Q_{\Lambda}\right)_{0} \mid P(x) \in \mathcal{A}\right\},\left(Q_{C}\right)_{0}=\left\{x \in\left(Q_{\Lambda}\right)_{0} \mid I(x) \in \mathcal{C}\right\}, I_{A}=I_{\Lambda} \cap k Q_{A}$ e $I_{C}=I_{\Lambda} \cap k Q_{C}$. Notemos também que $Q_{A}$ é fechada para sucessores e $Q_{C}$ é fechada para predecessores. Portanto $Q_{A}$ e $Q_{C}$ são também convexas em $Q_{\Lambda}$.

Consideremos $Q_{B}$ a subaljava plena de $Q_{\Lambda}$ tal que $\left(Q_{B}\right)_{0}=\left(Q_{A}\right)_{0} \cap\left(Q_{C}\right)_{0}$. Neste caso, $Q_{B}$ é também uma subaljava plena de $Q_{A}$ e de $Q_{C}$. Além disso, a convexidade de $Q_{A}$ e de $Q_{C}$ em $Q_{\Lambda}$ implica a convexidade de $Q_{B}$ em $Q_{\Lambda}$ e portanto em $Q_{A}$ e em $Q_{C}$. Defina o ideal $I_{B}$ por $I_{B}=I_{\Lambda} \cap k Q_{B}$. Neste caso, temos que $I_{A} \cap k Q_{B}=I_{B}=I_{C} \cap k Q_{C}$. Finalmente, seja $B=k Q_{B} / I_{B}$. Podemos então considerar o produto fibrado $R$ dos epimorfismos $A \rightarrow B$ e $C \rightarrow B$. Observe que tal produto fibrado é orientado pois $Q_{A}$ é fechada para sucessores e $Q_{C}$ para predecessores.

Mostraremos que $R$ é isomorfo a $\Lambda$ e, para tanto, mostraremos que $Q_{R}=Q_{\Lambda}$ e que $I_{R}=I_{\Lambda}$

- $\left(Q_{R}\right)_{0}=\left(Q_{\Lambda}\right)_{0}$ :

Sabemos que $\left(Q_{R}\right)_{0}=\left(Q_{A}\right)_{0} \cup\left(Q_{C}\right)_{0}$ e que $\left(Q_{A}\right)_{0} \cup\left(Q_{C}\right)_{0} \subseteq\left(Q_{\Lambda}\right)_{0}$. Se $x$ está em $\left(Q_{\Lambda}\right)_{0}$ e não está em $\left(Q_{A}\right)_{0}$ então o projetivo $P_{\Lambda}(x)$ não está em $\mathcal{A}$. Por hipótese $\Lambda$ está em $\operatorname{add}(\mathcal{A} \cup \mathcal{C}), \operatorname{logo} P_{\Lambda}(x)$ está em $\mathcal{C}$ e portanto $I_{\Lambda}(x)$ também está em $\mathcal{C}$, logo $x$ está em $\left(Q_{C}\right)_{0}$. Isto mostra que $\left(Q_{A}\right)_{0} \cup\left(Q_{C}\right)_{0}=\left(Q_{\Lambda}\right)_{0}$.

$\left(Q_{R}\right)_{1}=\left(Q_{\Lambda}\right)_{1}:$

Também temos que $\left(Q_{R}\right)_{1}=\left(Q_{A}\right)_{1} \cup\left(Q_{C}\right)_{1}$ e que $\left(Q_{A}\right)_{1} \cup\left(Q_{C}\right)_{1} \subseteq\left(Q_{\Lambda}\right)_{1}$. Como cada projetivo indecomponível está em $\mathcal{A} \cup \mathcal{C}$ então cada projetivo está em ind $A \cup \operatorname{ind} C$, e portanto o suporte de cada projetivo está ou em $Q_{A}$ ou em $Q_{C}$. Portanto não há flecha ligando $\left(Q_{A}\right)_{0} \backslash\left(Q_{C}\right)_{0}$ e $\left(Q_{C}\right)_{0} \backslash\left(Q_{A}\right)_{0}$, o que mostra que $\left(Q_{A}\right)_{1} \cup\left(Q_{C}\right)_{1}=\left(Q_{\Lambda}\right)_{1}$.

$I_{R}=I_{\Lambda}$ :

Sabemos que $I_{R}=I_{A}+I_{C}+I$ onde $I$ é o ideal gerado pelos caminhos que ligam $\left(Q_{A}\right)_{0} \backslash\left(Q_{B}\right)_{0}$ e $\left(Q_{C}\right)_{0} \backslash\left(Q_{B}\right)_{0}$. De fato, temos $I_{A}+I_{C} \subseteq I_{\Lambda}$. Além disso, como cada $\Lambda$-módulo projetivo está em $\mathcal{A} \cup \mathcal{C} \subseteq$ ind $A \cup$ ind $C$ então não existe caminho não nulo ligando $\left(Q_{A}\right)_{0} \backslash\left(Q_{B}\right)_{0}$ e $\left(Q_{C}\right)_{0} \backslash\left(Q_{B}\right)_{0}$, ou seja, $I$ também está em $I_{\Lambda}$. 
Por outro lado, consideremos $\omega$ em $I_{\Lambda}$. Observe que cada caminho está ou em $Q_{A}$ ou em $Q_{C}$ ou está em $I$. Se $\omega=e_{C} \omega e_{C}$ então $\omega$ está em $I_{\Lambda} \cap k Q_{C}=I_{C} \subseteq I_{R}$. Se $\omega=e_{A} \omega e_{A}$ então $\omega$ está em $I_{\Lambda} \cap k Q_{A}=I_{A} \subseteq I_{R}$. Se $\omega=e_{C}^{\prime} \omega e_{A}^{\prime}$ ou $\omega=e_{A}^{\prime} \omega e_{C}^{\prime}$ então $\omega$ está em $I \subseteq I_{R}$. Logo, $I_{A}+I_{C}+I=I_{\Lambda}$.

Portanto, $\Lambda$ é o produto fibrado orientado de $A \rightarrow B$ e $C \rightarrow B$.

Corolário 4.1.5 Seja $\Lambda=k Q_{\Lambda} / I_{\Lambda}$ uma álgebra ada. Então existe uma álgebra $B$ tal que $\Lambda$ é o produto fibrado orientado de epimorfismos ${ }_{\left(\mathcal{L}_{\Lambda}\right)} \Lambda \rightarrow B$ e $\Lambda_{\left(\mathcal{R}_{\Lambda}\right)} \rightarrow B$.

Observação 4.1.6 Lembremos que, se $\Lambda$ é uma álgebra quase inclinada então ela é uma álgebra ada. Se $\Lambda$ não é inclinada então não existe injetivo em $\mathcal{L}_{\Lambda}$ e consequentemente, todo injetivo indecomponível está em $\mathcal{R}_{\Lambda}$. Portanto, $\Lambda=\Lambda_{\left(\mathcal{R}_{\Lambda}\right)}$ e nesse caso o produto fibrado do Teorema 4.1.4 é trivial.

Se $\Lambda$ é uma álgebra shod estrita então por [CL99] existe um injetivo em $\mathcal{L}_{A} \backslash \mathcal{R}_{A}$ e neste caso o produto fibrado do Teorema 4.1.4 não é trivial.

Corolário 4.1.7 Sejam $\Lambda=k Q_{\Lambda} / I_{\Lambda}$ uma álgebra e $(\mathcal{A}, \mathcal{B}, \mathcal{C})$ uma trisecção para ind $\Lambda$ tal que $\Lambda \oplus D \Lambda$ está em $\operatorname{add}(\mathcal{A} \cup \mathcal{C})$. Então, existe $B$ tal que $\Lambda$ é o produto fibrado orientado de ${ }_{\mathcal{A}} \Lambda \rightarrow B$ e $\Lambda_{\mathcal{C}} \rightarrow B$.

Exemplo 4.1.8 Considere a álgebra $\Lambda$ com $\operatorname{rad}^{2} \Lambda=0$ cuja aljava ordinária é

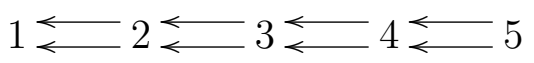

Note que não se trata de uma álgebra ada pois $I(2)$ e $P(4)$ não estão em $\mathcal{L}_{\Lambda} \cup \mathcal{R}_{\Lambda}$. Consideremos $\mathcal{C}=\operatorname{Succ} I(2)$ e $\mathcal{B}=\operatorname{ind} \Lambda \backslash\left(\mathcal{L}_{\Lambda} \cup \mathcal{C}\right)$. Nesse caso, $\left(\mathcal{L}_{\Lambda}, \mathcal{B}, \mathcal{C}\right)$ é uma trisecção para $\operatorname{ind} \Lambda$ e $\Lambda \oplus D \Lambda$ está em $\operatorname{add}\left(\mathcal{L}_{\Lambda} \cup \mathcal{C}\right)$. A álgebra suporte de $\mathcal{L}_{\Lambda}$ é dada por $1 \longleftarrow 2 \longleftarrow 3$ com $\operatorname{rad}^{2}\left({ }_{\left(\mathcal{L}_{\Lambda}\right)} \Lambda\right)=0$ e a álgebra suporte de $\mathcal{C}$ é dada por $2 \longleftarrow 3 \longleftarrow 4 \longleftarrow 5$ com $\operatorname{rad}^{2}\left(\Lambda_{\mathcal{C}}\right)=0$. Temos que $\Lambda$ é o produto fibrado orientado de ${ }_{\left(\mathcal{L}_{\Lambda}\right)} \Lambda \rightarrow B$ e $\Lambda_{\mathcal{C}} \rightarrow B$ onde $B$ é $2 \longleftarrow 3$.

Com a mesma demonstração de [ACLV12] (2.5) para as álgebras ada temos o seguinte resultado.

Proposição 4.1.9 Sejam $\Lambda=k Q_{\Lambda} / I_{\Lambda}$ uma álgebra e $\mathcal{A}$ e $\mathcal{C}$ subcategorias de ind $\Lambda$ tais que $\mathcal{A}$ é fechada para predecessores e $\mathcal{C}$ fechada para sucessores. Então $\Lambda \oplus D \Lambda$ está em $\operatorname{add}(\mathcal{A} \cup \mathcal{C})$ se, e somente se, $\operatorname{ind} \Lambda=\mathcal{A} \cup \operatorname{ind} \Lambda_{\mathcal{C}}=\operatorname{ind}_{\mathcal{A}} \Lambda \cup \mathcal{C}$. Em particular, se $\Lambda \oplus D \Lambda$ está em $\operatorname{add}(\mathcal{A} \cup \mathcal{C})$ então $\operatorname{ind} \Lambda=\operatorname{ind}_{\mathcal{A}} \Lambda \cup \operatorname{ind} \Lambda_{\mathcal{C}}$. 
Prova. Seja $M$ um $\Lambda$-módulo indecomponível que não está em ind $\Lambda_{\mathcal{C}}$. Então, existe $x$ no suporte de $M$ que não está em $Q_{\left(\Lambda_{\mathcal{C}}\right)}$. Neste caso, $I_{\Lambda}(x)$ não está em $\mathcal{C}$ e portanto $I_{\Lambda}(x)$ está em $\mathcal{A}$. Mas como $x$ está no suporte de $M$, existe um morfismo de $M$ em $I_{\Lambda}(x)$, logo $M$ também está em $\mathcal{A}$. A prova de que ind $\Lambda=\operatorname{ind}_{\mathcal{A}} \Lambda \cup \mathcal{C}$ é análoga.

Por outro lado, suponhamos que ind $\Lambda=\mathcal{A} \cup \operatorname{ind} \Lambda_{\mathcal{C}}=\operatorname{ind}_{\mathcal{A}} \Lambda \cup \mathcal{C}$ e seja $P(x)$ um projetivo indecomponível que não está em $\mathcal{A}$. Neste caso, $P(x)$ também não está em ind ${ }_{\mathcal{A}} \Lambda \mathrm{e}$ por ind $\Lambda=\operatorname{ind}_{\mathcal{A}} \Lambda \cup \mathcal{C}$ segue que $P(x)$ está em $\mathcal{C}$. Analogamente, mostra-se que os injetivos indecompoíveis também estão em $\mathcal{A} \cup \mathcal{C}$.

\subsection{Subcategorias especiais no produto fibrado}

Lembremos que para um produto fibrado orientado denotamos por $D A^{\prime}$ a soma direta dos injetivos indecomponíveis $I(x) \operatorname{com} x$ em $\left(Q_{A}\right)_{0} \backslash\left(Q_{B}\right)_{0}$ e por $C^{\prime}$ a soma direta dos projetivos indecomponíveis $P(x)$ com $x$ em $\left(Q_{C}\right)_{0} \backslash\left(Q_{B}\right)_{0}$. Consideremos as seguintes subcategorias de ind $R$ :

$$
\mathcal{A}=\operatorname{Pred} D A^{\prime}, \mathcal{C}=\operatorname{Succ} C^{\prime} \text { e } \mathcal{B}=\operatorname{ind} R \backslash(\mathcal{A} \cup \mathcal{C}) .
$$

Obviamente, $\mathcal{A}$ e $\mathcal{A} \backslash \mathcal{C}$ são fechadas para predecessores e $\mathcal{C}$ e $\mathcal{C} \backslash \mathcal{A}$ são fechadas para sucessores. Manteremos essa notação até o final desse capítulo.

Proposição 4.2.1 Seja $R$ o produto fibrado orientado de $A \rightarrow B e C \rightarrow B$. Então

$$
(\mathcal{A} \backslash \mathcal{C}, \mathcal{B} \cup(\mathcal{A} \cap \mathcal{C}), \mathcal{C} \backslash \mathcal{A})
$$

é uma trisecção de $\operatorname{ind} R$.

Prova. Pela definição de $\mathcal{B}$ temos que ind $R=\mathcal{A} \cup \mathcal{B} \cup \mathcal{C}$ e portanto ind $R=(\mathcal{A} \backslash \mathcal{C}) \cup$ $(\mathcal{B} \cup(\mathcal{A} \cap \mathcal{C})) \cup(\mathcal{C} \backslash \mathcal{A})$. Além disso, como $\mathcal{A} \backslash \mathcal{C}$ é fechada para predecessores então $\operatorname{Hom}(\mathcal{B} \cup(\mathcal{A} \cap \mathcal{C}), \mathcal{A} \backslash \mathcal{C})=0$ e $\operatorname{Hom}(\mathcal{C} \backslash \mathcal{A}, \mathcal{A} \backslash \mathcal{C})=0$. Também, como $\mathcal{C} \backslash \mathcal{A}$ é fechada para sucessores segue que $\operatorname{Hom}(\mathcal{C} \backslash \mathcal{A}, \mathcal{B} \cup(\mathcal{A} \cap \mathcal{C}))=0$.

Vejamos alguma propriedades das categorias $\mathcal{A}$ e $\mathcal{C}$.

Lema 4.2.2 Seja $R$ o produto fibrado orientado de $A \rightarrow B$ e $C \rightarrow B$. Então,

i) $\operatorname{ind} A \backslash \operatorname{ind} B \subseteq \mathcal{A}$,

ii) $\operatorname{ind} C \backslash \operatorname{ind} B \subseteq \mathcal{C}$,

iii) $\operatorname{ind} R \backslash(\operatorname{ind} A \cup \operatorname{ind} C) \subseteq \mathcal{A} \cap \mathcal{C}$,

iv) $\mathcal{B} \subseteq \operatorname{ind} B$, 
v) $\mathcal{A} \backslash \mathcal{C} \subseteq \operatorname{ind} A$,

vi) $\mathcal{C} \backslash \mathcal{A} \subseteq \operatorname{ind} C$

Prova.

i) Se $M \in \operatorname{ind} A \backslash \operatorname{ind} B$ então existe $x$ de $\left(Q_{A}\right)_{0} \backslash\left(Q_{B}\right)_{0}$ que está no suporte de $M$. Logo, existe um morfismo $M \rightarrow I(x)$ e portanto $M$ está em $\operatorname{Pred} D A^{\prime}=\mathcal{A}$.

ii) Análogo ao item anterior.

iii) Se $M \in \operatorname{ind} R \backslash(\operatorname{ind} A \cup \operatorname{ind} C)$ então no suporte de $M$ existem $y$ de $\left(Q_{A}\right)_{0} \backslash\left(Q_{B}\right)_{0}$ e $x$ de $\left(Q_{C}\right)_{0} \backslash\left(Q_{B}\right)_{0}$. Logo, existem morfismos $P(x) \rightarrow M$ e $M \rightarrow I(y)$ e portanto $M$ está em $\mathcal{A} \cap \mathcal{C}$.

iv) Se $M \in \mathcal{B}$ então $M$ não está em $\mathcal{A} \cup \mathcal{C}$. Pelos itens (i) e (ii), segue que $M$ não está em (ind $A \cup \operatorname{ind} C) \backslash \operatorname{ind} B$ e pelo item (iii) segue que $M$ não está em $\operatorname{ind} R \backslash(\operatorname{ind} A \cup \operatorname{ind} C$ ). Portanto, $M$ está em ind $B$.

v) Se $M \in \mathcal{A} \backslash \mathcal{C}$ então $M$ não está em $\mathcal{A} \cap \mathcal{C}$ e pelo item (iii) segue que $M$ está em ind $A \cup \operatorname{ind} C$. Por outro lado, pelo item (ii), $M$ não está em ind $C \backslash \operatorname{ind} B$. Logo $M$ está em $\operatorname{ind} A$.

vi) Análogo ao item anterior.

Corolário 4.2.3 Seja $R$ o produto fibrado orientado de $A \rightarrow B$ e $C \rightarrow B$. Então,

$$
\operatorname{ind} R=\mathcal{A} \cup \operatorname{ind} C=\operatorname{ind} A \cup \mathcal{C} \text {. }
$$

Prova. De fato, pela definição de $\mathcal{B}$, temos que ind $R$ é $\mathcal{A} \cup \mathcal{C} \cup \mathcal{B}$. Do item vi) do lema anterior temos que $\mathcal{C} \backslash \mathcal{A}$ está em ind $C$ e do item iv) temos que $\mathcal{B}$ está também em ind $C$. Portanto, $\operatorname{ind} R=\mathcal{A} \cup \operatorname{ind} C$. A outra igualdade é análoga.

Corolário 4.2.4 Seja $R$ o produto fibrado orientado de $A \rightarrow B$ e $C \rightarrow B$. Se $\mathcal{A}$ e $\mathcal{C}$ são disjuntas então $\operatorname{ind} R=\operatorname{ind} A \cup \operatorname{ind} C$.

Prova. Do item iii) do Lema 4.2.2 temos que ind $R \backslash($ ind $A \cup \operatorname{ind} C$ ) está em $\mathcal{A} \cap \mathcal{C}$ que é vazio. Logo, ind $R=\operatorname{ind} A \cup \operatorname{ind} C$. 


\subsection{Aljava de Auslander-Reiten do produto fibrado orientado}

Vimos que, para um produto fibrado Dynkin orientado, os $R$-módulos indecomponíveis são justamente aqueles de ind $A$ e de ind $C$. É natural, portanto, verificar qual a relação entre as aljavas de Auslander-Reiten dessas álgebras.

Em [Lev04], Lévesque utiliza o seguinte resultado para um produto fibrado Nakayama orientado:

Lema 4.3.1 ([Lev04](3.2.2)) Seja $R$ o produto fibrado Nakayama orientado de $A \rightarrow B$ e $C \rightarrow B$.

(a) Se $M$ é um $C$-módulo indecomponível em $\operatorname{ind} C \backslash \operatorname{ind} B$ então o suporte do topo de $M$, $\operatorname{supp}(\operatorname{top} M)$, está em $\left(Q_{C}\right)_{0} \backslash\left(Q_{B}\right)_{0}$.

(b) Se $M$ é um A-módulo indecomponivel em ind $A \backslash \operatorname{ind} B$ então o suporte do socle de $M$, $\operatorname{supp}(\operatorname{soc} M)$, está em $\left(Q_{A}\right)_{0} \backslash\left(Q_{B}\right)_{0}$.

A partir disso, Lévesque mostrou que, se $R$ é um o produto fibrado Nakayama orientado de $A \rightarrow B$ e $C \rightarrow B$, então a aljava de Auslander-Reiten $\Gamma_{B}$ da álgebra $B$ é uma subaljava plena de $\Gamma_{A}$ e de $\Gamma_{C}$ e estas são subaljavas plenas de $\Gamma_{R}$. Mais ainda, ela mostra que $\Gamma_{R}$ é uma soma amalgamada das inclusões $\Gamma_{B} \rightarrow \Gamma_{A}$ e $\Gamma_{B} \rightarrow \Gamma_{C}$, isto é, $\Gamma_{R}=\Gamma_{A} \coprod_{\Gamma_{B}} \Gamma_{C}$.

O próximo exemplo mostra que o mesmo resultado não vale para o produto fibrado Dynkin orientado.

Exemplo 4.3.2 Consideremos as álgebras $A, B$ e $C$ dadas, respectivamente, pelas aljavas

$$
Q_{A}=\begin{array}{rr}
1 \leftarrow 3 \\
\downarrow
\end{array} \quad Q_{B}=\begin{aligned}
& 3 \\
& \downarrow \\
& 2
\end{aligned} \quad \begin{aligned}
& 2 \\
& 2
\end{aligned} \quad \begin{aligned}
& 3 \leftarrow 4 \\
& \downarrow \\
& 2
\end{aligned}
$$

Nesse caso, o produto fibrado $R$ é dado por $\begin{array}{r}1<-\overline{3}<4 \\ \downarrow \\ 2\end{array}$, que é um produto fibrado que satisfaz as condições * da Definição 3.3 .1 e, portanto, é também um produto fibrado Dynkin orientado.

$O$ simples $S(2)$ está no socle do $A$-módulo $P(3)={ }_{1}^{3}{ }_{2} \in \operatorname{ind} A \backslash \operatorname{ind} B$. Vejamos o que acontece com as aljavas de Auslander-Reiten. Temos 

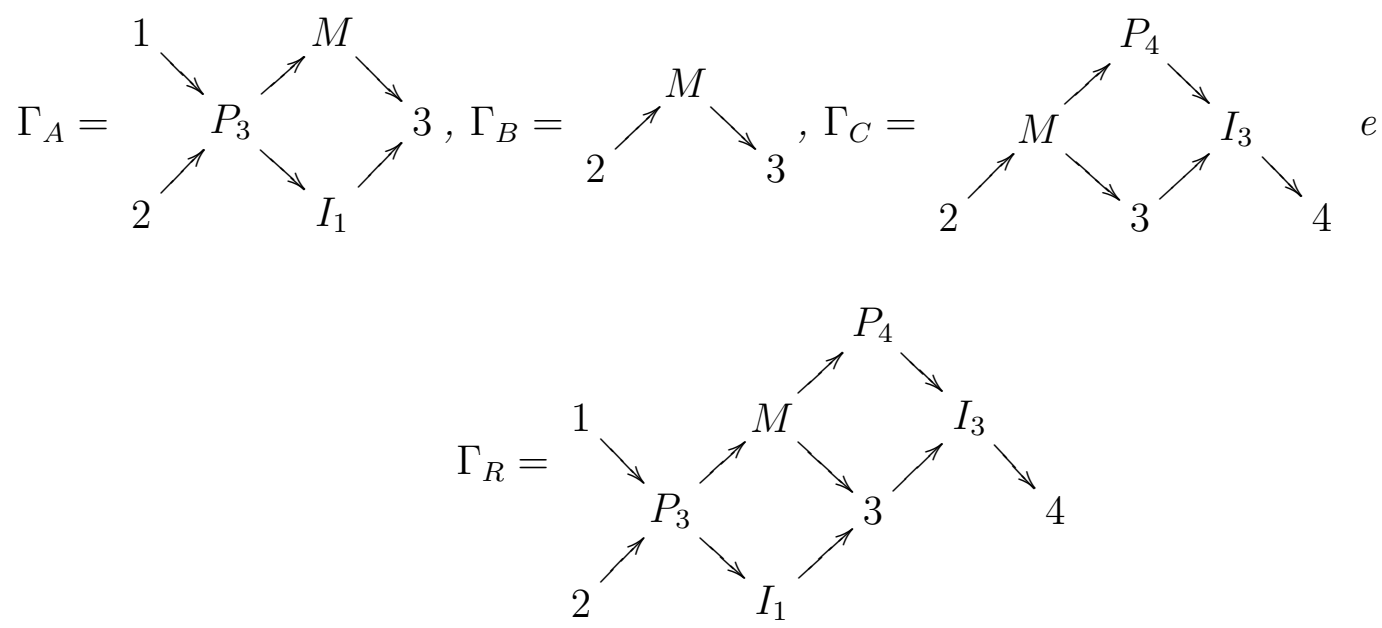

Observe que $\Gamma_{B}$ não é uma subaljava de $\Gamma_{A}$ e, portanto, nãa vale $\Gamma_{R}=\Gamma_{A} \coprod_{\Gamma_{B}} \Gamma_{C}$.

Proposição 4.3.3 Se $R$ é o produto fibrado Nakayama orientado de $A \rightarrow B$ e $\rightarrow B$ então

(a) $\operatorname{Hom}(\operatorname{ind} C, \operatorname{ind} A \backslash \operatorname{ind} B)=0$

(b) $\operatorname{Hom}(\operatorname{ind} C \backslash \operatorname{ind} B$, ind $A)=0$

Prova.

(a) Sejam $M$ em ind $C$ e $N$ em ind $A \backslash \operatorname{ind} B$. Suponha $f$ em $\operatorname{Hom}(M, N)$ um morfismo não nulo. Então, $\operatorname{im} f$ é um submódulo não nulo de $N$ e portanto $\operatorname{soc} N \cap \operatorname{im} f$ é ainda não nulo.

Seja $S(x)$ um módulo simples em $\operatorname{soc} N \cap \operatorname{im} f$. Então, $S(x)$ é fator de composição de $\operatorname{im} f \cong M / \operatorname{ker} f$ e portanto é também fator de composiçào de $M$, ou seja, $x$ está em $\left(Q_{C}\right)_{0}$. Por outro lado, por (b) do Lema 4.3.1, temos que $x$ está em $\left(Q_{A}\right)_{0} \backslash\left(Q_{B}\right)_{0}$, uma contradição. $\operatorname{Logo} \operatorname{Hom}(M, N)=0$.

(b) Sejam $M$ em $\operatorname{ind} C \backslash \operatorname{ind} B$ e $N$ em ind $A$. Suponha $f$ em $\operatorname{Hom}(M, N)$ um morfismo não nulo. Se $(P(M), p)$ é a cobertura projetiva de $M$ então $f \circ p: P(M) \rightarrow N$ é ainda não nulo pois $p$ é um epimorfismo. Seja $(P(N), h)$ a cobertura projetiva de $N$, então existe um morfismo $u: P(M) \rightarrow P(N)$ tal que $h \circ u=f \circ p$. Observe que $u$ é não nulo, então existem $i \operatorname{em~} \operatorname{supp}(\operatorname{top} M), j$ em $\operatorname{supp}(\operatorname{top} N)$ e um morfismo não nulo $P(i) \rightarrow P(j)$. Nesse caso existe um caminho não nulo de $j$ para $i$. Mas, $j$ está em $\left(Q_{B}\right)_{0}$ e pelo item (a) do Lema 4.3.1 temos $i$ em $\left(Q_{C}\right)_{0} \backslash\left(Q_{B}\right)_{0}$, uma contradição com a orientação da aljava.

Observemos que ind $A$ é uma categoria fechada para predecessores pois se $M$ é um $A$ módulo indecomponível e $f: N \rightarrow M$ é um morfismo não nulo então $N \in \operatorname{ind} R=\operatorname{ind} A \cup \operatorname{ind} C$ 
não está em ind $C \backslash \operatorname{ind} B$, pois $\operatorname{Hom}(\operatorname{ind} C \backslash \operatorname{ind} B$, ind $A)=0$, e portanto $N$ também está em ind $A$. Dualmente, ind $C$ é fechada para sucessores.

Corolário 4.3.4 Se $R$ é o produto fibrado Nakayama orientado de $A \rightarrow B$ e $C \rightarrow B$ então ind $A$ é fechada para predecessores e ind $C$ é fechada para sucessores.

Segue, então, que para o produto fibrado Nakayama orientado existe uma trisecção para ind $R$ :

Corolário 4.3.5 Se $R$ é o produto fibrado Nakayama orientado de $A \rightarrow B$ e $C \rightarrow B$ então (ind $A \backslash \operatorname{ind} B$, ind $B$, ind $C \backslash \operatorname{ind} B$ ) é uma trisecção de $\operatorname{ind} R$.

Lembremos as subcategorias consideradas nas últimas seções para um produto fibrado orientado $R$ dos epimorfismos $A \rightarrow B$ e $C \rightarrow B$ :

$$
\mathcal{A}=\operatorname{Pred} D A^{\prime}, \mathcal{C}=\operatorname{Succ} C^{\prime} \text { e } \mathcal{B}=\operatorname{ind} R \backslash(\mathcal{A} \cup \mathcal{C})
$$

Por abuso de notação escreveremos, em alguns casos, $\mathcal{X}$ no lugar de 'objetos de $\mathcal{X}$ '.

Veremos que, para um produto fibrado orientado com $\mathcal{A}$ e $\mathcal{C}$ disjuntas, podemos escrever a aljava de Auslander-Reiten $\Gamma_{R}$ como uma soma amalgamada $\Sigma_{A} \coprod_{\Sigma_{B}} \Sigma_{C}$ onde $\Sigma_{A}, \Sigma_{B}$ e $\Sigma_{C}$ são subaljavas plenas de $\Gamma_{A}, \Gamma_{B}$ e $\Gamma_{C}$ respectivamente.

Lema 4.3.6 Seja $R$ o produto fibrado orientado de $A \rightarrow B$ e $C \rightarrow B$. Considere $S=A, B$ ou $C$ e $f: M \rightarrow N$ um morfismo com $M$ e $N$ dois $S$-módulos.

(a) $f$ é um monomorfismo que cinde em $\bmod S$ se, e somente se, $f$ é um monomorfismo que cinde em $\bmod R$;

(b) $f$ é um epimorfismo que cinde em $\bmod S$ se, e somente se, $f$ é um epimorfismo que cinde em $\bmod R$.

Prova. Segue da plenitude de $\bmod S$ em $\bmod R$.

Seja $R$ o produto fibrado orientado de $A \rightarrow B$ e $C \rightarrow B$ e suponhamos que $\mathcal{A}$ e $\mathcal{C}$ são disjuntas. Nesse caso, da Proposição 4.2.1 segue que $(\mathcal{A}, \mathcal{B}, \mathcal{C})$ é uma trisecção de ind $R$.

Consideremos

- $\Sigma_{A}$ a subaljava plena de $\Gamma_{R}$ cujo conjunto de vértices é o mesmo dos objetos de $\mathcal{A} \cup \mathcal{B}$;

- $\Sigma_{C}$ a subaljava plena de $\Gamma_{R}$ cujo conjunto de vértices é o mesmo dos objetos de $\mathcal{B} \cup \mathcal{C}$; e

- $\Sigma_{B}$ a subaljava plena de $\Gamma_{R}$ cujo conjunto de vértices é o mesmo dos objetos de $\mathcal{B}$. 
Então, $\Sigma_{B}$ é uma subaljava plena de $\Sigma_{A}$ e de $\Sigma_{C}$. Temos que $\left(\Gamma_{R}\right)_{0}=\left(\Sigma_{A}\right)_{0} \cup\left(\Sigma_{C}\right)_{0}$ e $\left(\Sigma_{B}\right)_{0}=\left(\Sigma_{A}\right)_{0} \cap\left(\Sigma_{C}\right)_{0}$. Da proposição 3.1.13, é fácil ver que $\Gamma_{R}$ é a soma amalgamada das inclusões $\Sigma_{B} \rightarrow \Sigma_{A}$ e $\Sigma_{B} \rightarrow \Sigma_{C}$.

Lema 4.3.7 Consideremos $\Sigma_{A}, \Sigma_{B}$ e $\Sigma_{C}$ como construído acima. Então,

(a) $\Sigma_{B}$ é uma subaljava plena de $\Gamma_{B}, \Gamma_{A}$ e $\Gamma_{C}$;

(b) $\Sigma_{A}$ é uma subaljava plena de $\Gamma_{A}$; e

(c) $\Sigma_{C}$ é uma subaljava plena de $\Gamma_{C}$

Prova. Seja $S=B, A$ ou $C$. Pela construção temos que $\left(\Sigma_{B}\right)_{0}=\mathcal{B} \subseteq \operatorname{ind} B \subseteq \operatorname{ind} S=\left(\Gamma_{S}\right)_{0}$. Vejamos agora que cada flecha em $\Sigma_{B}$ corresponde a uma flecha em $\Gamma_{S}$. Seja $f \in\left(\Sigma_{B}\right)_{1}$, então $f: M \rightarrow N$ é um morfismo irredutível em $\bmod R \operatorname{com} M$ e $N$ em $\mathcal{B}$. Vejamos que $f$ é também um morfismo irredutível em $\bmod S$.

Pelo Lema 4.3.6, $f$ não é monomorfismo que cinde e nem epimorfismo que cinde em $\bmod S$. sejam $L \in \bmod S, h: M \rightarrow L, g: L \rightarrow N$ tais que $f=g h$. Pela plenitude de $\bmod S$ em $\bmod R$ segue que $g$ e $h$ são $\operatorname{morfismos}$ em $\bmod R$, e portanto, pelo Lema 4.3.6, segue que $h$ é um monomorfismo que cinde em $\bmod S$ ou $g$ é um epimorfismo que cinde em mod $S$. Com isso, mostramos que $\Sigma_{B}$ é uma subaljava de $\Gamma_{S}$.

Para a plenitude, seja $f: M \rightarrow N$ uma flecha em $\Gamma_{S}$ com $M$ e $N$ dois módulos em $\mathcal{B}$. Queremos mostrar que $f$ é uma flecha em $\Sigma_{B}$, ou seja, um morfismo irredutível em mod $R$. Novamente, pelo Lema 4.3.6, $f$ não é monomorfismo que cinde e nem epimorfismo que cinde. Sejam $h: M \rightarrow L$ e $g: L \rightarrow N$ morfismos em $\bmod R$ tais que $f=g h$. Como $M$ e $N$ estão em $\mathcal{B}$ que é uma subcategoria convexa de ind $R$ segue que $L$ também está em $\bmod B \subseteq \bmod S$. Pela plenitude de $\bmod S$ em $\bmod R$ segue que $g$ e $h$ são morfismos em $\bmod S$ e, portanto, $h$ é um monomorfismo que cinde em $\bmod S$ ou $g$ é um epimorfismo que cinde em $\bmod R$. Isso mostra (a).

A prova de (b) e de (c) são análogas, uma vez que $\left(\Sigma_{A}\right)_{0}$ e $\left(\Sigma_{C}\right)_{0}$ são subcategorias convexas de ind $R$ e que $\bmod A$ e $\bmod C$ são subcategorias plenas de $\bmod R$.

Com isso, podemos enunciar o seguinte resultado.

Teorema 4.3.8 Seja $R$ o produto fibrado orientado de $A \rightarrow B$ e $C \rightarrow B$ e suponha que $\mathcal{A}$ e $\mathcal{C}$ são disjuntas. Então, existem $\Sigma_{A}$ uma subaljava plena de $\Gamma_{A}, \Sigma_{B}$ uma subaljava plena de $\Gamma_{B}$ e $\Sigma_{C}$ uma subaljava plena de $\Gamma_{C}$ tais que $\Gamma_{R}$ é a soma amalgamada $\Sigma_{A} \coprod_{\Sigma_{B}} \Sigma_{C}$ das inclusões $\Sigma_{B} \rightarrow \Sigma_{A}$ e $\Sigma_{B} \rightarrow \Sigma_{C}$.

Exemplo 4.3.9 No Exemplo 4.3.2, temos

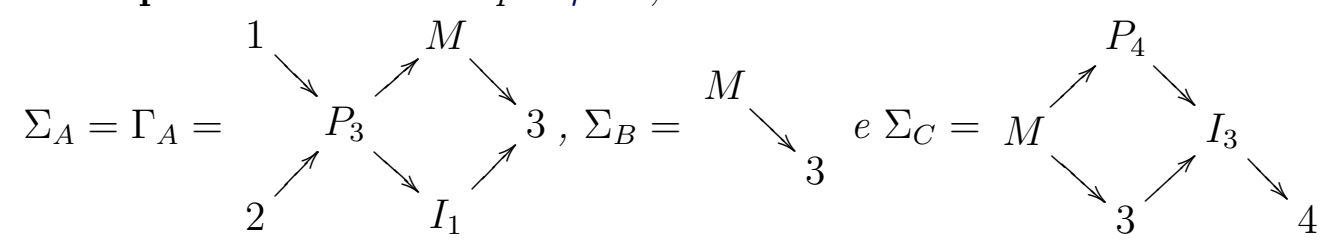




\subsection{Produto fibrado orientado e classes de algebras}

Vimos na seção 1.4 do Capítulo 1 algumas classes de álgebras que podem ser determinadas a partir das partes direita e esquerda da categoria de módulos. Nesta seção gostaríamos de relacionar as classes das álgebras envolvidas em um produto fibrado.

Proposição 4.4.1 Seja $R$ o produto fibrado de $A \rightarrow B$ e $C \rightarrow B$. Então,

a) Se $R$ é uma álgebra laura, então $A, B$ e $C$ também são.

b) Se $R$ é uma colagem à esquerda (ou à direita) então $A, B$ e $C$ também são.

c) Se $R$ é shod então $A, B$ e $C$ também são.

d) Se $R$ é quase inclinada então $A, B$ e $C$ também são.

e) Se $R$ é ada então $A, B$ e $C$ também são.

Prova. Segue do fato de que para um produto fibrado qualquer tem-se $A=e_{A} R e_{A}, B=$ $e_{B} R e_{B}$ e $C=e_{C} R e_{C}$ aplicando [AC04] para os 4 primeiros itens e [ACLV12](2.8) para o caso ada.

Para analisar o sentido contrário dessas implicações vamos, primeiramente, relacionar as partes direita e esquerda do produto fibrado $R$ com as respectivas partes direita e esquerda das álgebras $A$ e $C$.

Lema 4.4.2 Seja $R$ o produto fibrado orientado de $A \rightarrow B$ e $C \rightarrow B$. Então,

$$
\mathcal{L}_{A} \cap(\mathcal{A} \backslash \mathcal{C}) \subseteq \mathcal{L}_{R} \text { e } \mathcal{R}_{C} \cap(\mathcal{C} \backslash \mathcal{A}) \subseteq \mathcal{R}_{R}
$$

Prova. Faremos a primeira inclusão, pois a segunda é dual. Sejam $M$ um $A$-módulo em $\mathcal{L}_{A} \cap(\mathcal{A} \backslash \mathcal{C})$ e $N \in \operatorname{ind} R$ um predecessor de $M$. Seja $\phi$ um caminho de $N$ para $M$ em ind $R$. Como $\mathcal{A} \backslash \mathcal{C}$ é fechada para predecessores, segue que cada módulo em $\phi$ está em $\mathcal{A} \backslash \mathcal{C}$ e portanto $\phi$ é um caminho em $\operatorname{ind} A$. Logo, $\operatorname{dp}_{A} N \leq 1$. Pela Proposição 3.2.10, segue que $\operatorname{dp} N=\operatorname{dp}_{A} N \leq 1$ e portanto $M$ está em $\mathcal{L}_{R}$.

Proposição 4.4.3 Seja $R$ o produto fibrado orientado de $A \rightarrow B$ e $\rightarrow B$. Suponhamos que $\mathcal{A} \cap \mathcal{C}$ é uma subcategoria finita.

(a) Se A é uma colagem à direita e $C$ é de tipo finito então $R$ é também uma colagem à direita.

(b) Se C é uma colagem à esquerda e A é de tipo finito então $R$ é também uma colagem à esquerda. 
(c) Se A é uma colagem à direita, $C$ é uma colagem à esquerda e $\mathcal{B}$ é finita então $R$ é laura.

Prova.

(a) Se $A$ é uma colagem à direita então ind $A \backslash \mathcal{L}_{A}$ é finita. Como ind $C$ é finita, pelo Lema 4.2.2, temos que $\mathcal{B} \cup(\mathcal{C} \backslash \mathcal{A})$ é finita. Do último lema, segue que $\operatorname{ind} R \backslash \mathcal{L}_{R} \subseteq$ $\operatorname{ind} R \backslash\left(\mathcal{L}_{A} \cap(\mathcal{A} \backslash \mathcal{C})\right)$. Lembremos que pela definição de $\mathcal{A}$ e $\mathcal{C}$ temos

$$
\operatorname{ind} R=(\mathcal{A} \backslash \mathcal{C}) \cup \mathcal{B} \cup(\mathcal{A} \cap \mathcal{C}) \cup(\mathcal{C} \backslash \mathcal{A})
$$

Como $\mathcal{B} \cup(\mathcal{A} \cap \mathcal{C}) \cup(\mathcal{C} \backslash \mathcal{A})$ é finita, falta ver que $(\mathcal{A} \backslash \mathcal{C}) \backslash\left(\mathcal{L}_{\mathcal{A}} \cap(\mathcal{A} \backslash \mathcal{C})\right)=(\mathcal{A} \backslash \mathcal{C}) \backslash \mathcal{L}_{\mathcal{A}}$ é também finita. De fato, pelo Lema 4.2.2, $(\mathcal{A} \backslash \mathcal{C}) \backslash \mathcal{L}_{A} \subseteq$ ind $A \backslash \mathcal{L}_{A}$ que é finita. Com isso, mostramos que ind $R \backslash \mathcal{L}_{R}$ é finita e portanto $R$ é uma colagem à direita.

(b) Dual de (a).

(c) Para mostrar que $R$ é laura, basta mostrar que ind $R \backslash\left(\mathcal{L}_{R} \cup \mathcal{R}_{R}\right)$ é finita. Pelo último lema, temos

$$
\begin{aligned}
& \operatorname{ind} R \backslash\left(\mathcal{L}_{R} \cup \mathcal{R}_{R}\right) \subseteq \operatorname{ind} R \backslash\left(\left(\mathcal{L}_{A} \cap(\mathcal{A} \backslash \mathcal{C})\right) \cup\left(\mathcal{R}_{C} \cap(\mathcal{C} \backslash \mathcal{A})\right)\right)= \\
& \quad \operatorname{ind} R \backslash\left(\mathcal{L}_{A} \cap(\mathcal{A} \backslash \mathcal{C})\right) \cap \operatorname{ind} R \backslash\left(\mathcal{R}_{C} \cap(\mathcal{C} \backslash \mathcal{A})\right)
\end{aligned}
$$

Mas,

- $\operatorname{ind} R \backslash\left(\mathcal{L}_{A} \cap(\mathcal{A} \backslash \mathcal{C})\right)=\left((\mathcal{A} \backslash \mathcal{C}) \backslash \mathcal{L}_{A}\right) \cup \mathcal{B} \cup \mathcal{C} \mathrm{e}$

- ind $R \backslash\left(\mathcal{R}_{C} \cap(\mathcal{C} \backslash \mathcal{A})\right)=\mathcal{A} \cup \mathcal{B} \cup\left((\mathcal{C} \backslash \mathcal{A}) \backslash \mathcal{R}_{C}\right)$.

Portanto,

$$
\operatorname{ind} R \backslash\left(\mathcal{L}_{R} \cup \mathcal{R}_{R}\right) \subseteq\left((\mathcal{A} \backslash \mathcal{C}) \backslash \mathcal{L}_{A}\right) \cup \mathcal{B} \cup(\mathcal{A} \cap \mathcal{C}) \cup\left((\mathcal{C} \backslash \mathcal{A}) \backslash \mathcal{R}_{\mathcal{C}}\right)
$$

Como $A$ é uma colagem à direita e $C$ é uma colagem à esquerda, segue que $(\mathcal{A} \backslash \mathcal{C}) \backslash \mathcal{L}_{A} \subseteq$ $\operatorname{ind} A \backslash \mathcal{L}_{A}$ e $(\mathcal{C} \backslash \mathcal{A}) \backslash \mathcal{R}_{C} \subseteq \operatorname{ind} C \backslash \mathcal{R}_{C}$ são finitas. Finalmente, por hipótese, temos que $\mathcal{B} \cup(\mathcal{A} \cap \mathcal{C})$ também é finita e portanto ind $R \backslash\left(\mathcal{L}_{R} \cup \mathcal{R}_{R}\right)$ é finita.

Teorema 4.4.4 Seja $R$ o produto fibrado orientado de $A \rightarrow B$ e $C \rightarrow B$ e suponha que $\mathcal{A}$ e $\mathcal{C}$ são disjuntas. Se $A$ e $C$ são hereditárias então $R$ é inclinada.

Prova. Como $\mathcal{A}$ e $\mathcal{C}$ são disjuntas, pelo Corolário 4.2.4, temos que ind $R=\operatorname{ind} A \cup \operatorname{ind} C$. Pela Proposição 3.2.10 segue que $R$ é uma álgebra shod, pois $A$ e $C$ são álgebras hereditárias. 
Do Corolário 3.2.14 temos que dim.gl $R \leq 2$. Para ver que $R$ é uma álgebra inclinada, pelo Teorema 1.4.2, basta ver que a parte esquerda $\mathcal{L}_{R}$ contém algum injetivo. De fato, como $\mathcal{A}$ e $\mathcal{C}$ são disjuntas então $\mathcal{A}=\mathcal{A} \backslash \mathcal{C} \subseteq$ ind $A$ e como $\mathcal{A}$ é fechada para predecessores e $A$ é hereditária, segue que $\mathcal{A}$ está em $\mathcal{L}_{R}$. Finalmente, se $x$ é um vértice em $\left(Q_{A}\right)_{0} \backslash\left(Q_{B}\right)_{0}$ então o injetivo $I_{R}(x)$ está em $\mathcal{A}$ e portanto em $\mathcal{L}_{R}$.

Em particular, isso nos dá uma nova demonstração para [Lev08] (2.4.7) que é quando o produto fibrado é Nakayama orientado.

Corolário 4.4.5 Seja $R$ o produto fibrado Nakayama orientado de $A \rightarrow B$ e $C \rightarrow B$. Se $A$ e $C$ são álgebras hereditárias então $R$ é uma álgebra inclinada.

Prova. Cada somando de $D A^{\prime}$ está em ind $A \backslash \operatorname{ind} B$ que, pelo Corolário 4.3.4, é fechada para predecessores. Portanto, $\mathcal{A}$ está em ind $A \backslash \operatorname{ind} B$. Analogamente, vemos que $\mathcal{C}$ está em $\operatorname{ind} C \backslash \operatorname{ind} B$. Portanto, $\mathcal{A}$ e $\mathcal{C}$ são disjuntas.

Exemplo 4.4.6 Considere as álgebras $A, B$ e $C$ dadas, respectivamente, pelas seguintes

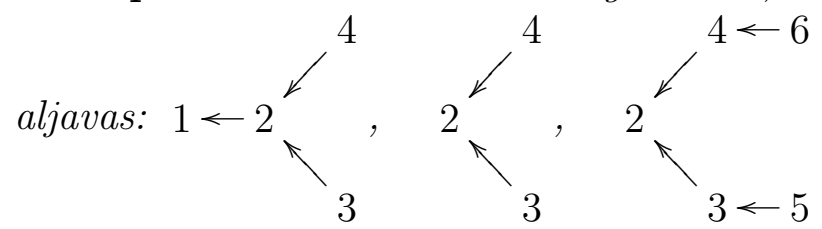

Nesse caso, o produto fibrado orientado dos epimorfismos $A \rightarrow B$ e $C \rightarrow B$ é a álgebra $R$ dada por

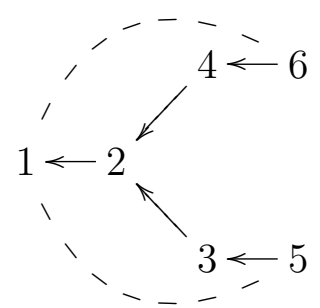

que é uma álgebra inclinada, pois $\mathcal{A}$ e $\mathcal{C}$ são disjuntas e $A$ e $C$ são hereditárias. Observe que não se trata de um produto fibrado Dynkin orientado, pois não satisfaz o item (b) da definição.

Exemplo 4.4.7 Considere as álgebras $A, B$ e $C$ dadas, respectivamente, pelas seguintes aljavas com relações: $1 \leftarrow 2 \swarrow_{3}^{4}, 2 \swarrow_{3}^{4}$

Nesse caso, o produto fibrado Dynkin orientado dos epimorfismos $A \rightarrow B$ e $C \rightarrow B$ é a álgebra $R$ dada por

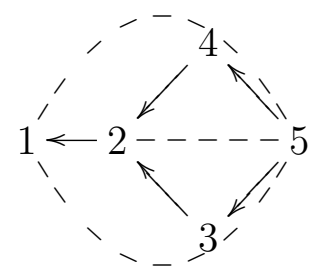


que é uma álgebra inclinada. Observe que nesse caso $\mathcal{A}$ e $\mathcal{C}$ são disjuntas e que $C$ não é hereditária.

Lembremos que um módulo $M$ é dito convexo se dados $M^{\prime}$ e $M^{\prime \prime}$ indecomponíveis em $\operatorname{add} M$ e um caminho em ind $R$ de $M^{\prime}$ para $M^{\prime \prime}$ :

$$
M^{\prime} \rightarrow X_{1} \rightarrow X_{2} \rightarrow \cdots \rightarrow X_{t} \rightarrow M^{\prime \prime}
$$

então cada $X_{i}$ também está em add $M$.

Teorema 4.4.8 Seja $R$ o produto fibrado orientado de $A \rightarrow B$ e $\rightarrow B$ com $A$ e $C$ álgebras hereditárias. Se $C^{\prime}$ ou $D A^{\prime}$ for um módulo convexo em ind $R$ então $R$ é uma álgebra inclinada.

Prova. Do Corolário 3.2.14 temos que dim.gl $R \leq 2$. Suponhamos que $D A^{\prime}$ seja um módulo convexo. Mostraremos que $\mathcal{A}$ está em $\mathcal{L}_{R}$. Para isso basta ver que, para cada $i$ de $\left(Q_{A}\right)_{0} \backslash$ $\left(Q_{B}\right)_{0}$, o injetivo $I(i)$ está em $\mathcal{L}_{R}$. Como cada $I(i)$ está em ind $A$ segue da Proposição 3.2.10 que $\operatorname{dp} D A^{\prime} \leq 1$. Seja $M$ um predecessor de $I(i)$ para algum $i$ de $\left(Q_{A}\right)_{0} \backslash\left(Q_{B}\right)_{0}$ e suponhamos que $\operatorname{dp} M \geq 2$. Neste caso, $\operatorname{Hom}_{R}(D R, \tau M) \neq 0$ (ver por exemplo [ASS06] IV.2.7) e portanto existe $t$ em $\left(Q_{R}\right)_{0}$ e um morfismo $I(t) \rightarrow \tau M$. Logo existe um caminho, de $I(t)$ para $I(i)$ : $I(t) \rightarrow \tau M \rightsquigarrow M \rightsquigarrow I(i)$. Como $i$ está em $\left(Q_{A}\right)_{0} \backslash\left(Q_{B}\right)_{0}$ que é fechada para sucessores segue que $t$ também está em $\left(Q_{A}\right)_{0} \backslash\left(Q_{B}\right)_{0}$. Uma contradição com a convexidade de $D A^{\prime}$. Logo, $\operatorname{dp} M \leq 1$ e portanto $\mathcal{A} \subseteq \mathcal{L}_{R}$.

Segue então que existem injetivos em $\mathcal{L}_{R}$ e também que $R$ é shod, pois se $M$ está em ind $A \cup$ ind $C$ já tinhamos, pela Proposição 3.2.10, que $\operatorname{dp} M \leq 1$ ou di $M \leq 1$ e se $M$ está em $\operatorname{ind} R \backslash(\operatorname{ind} A \cup \operatorname{ind} C$ ) então $M$ está em $\mathcal{A}$ e pela afirmação acima $\operatorname{dp} M \leq 1$.

O resultado segue agora do Teorema 1.4.2.

Ao supormos que $C^{\prime}$ é convexo, mostrariamos que $\mathcal{C}$ está em $\mathcal{R}_{R}$ e a conclusão se dá de forma análoga.

Conjecturamos que para um produto fibrado orientado se $A$ é hereditária, então $D A^{\prime}$ é convexo e se $C$ é hereditária então $C^{\prime}$ é convexo. Isso nos daria, pelo último resultado uma generalização para o Teorema 4.4.4. 


\section{Capítulo 5}

\section{Dimensão de representação}

Neste capítulo, $\Lambda$ denotará uma álgebra de Artin. Porém, quando tratarmos de produtos fibrados estaremos nos restringindo às notações do capítulo precedente, ou seja, álgebras de dimensão finita sobre o corpo $k$. Em qualquer dos casos, os módulos são finitamente gerados à direita.

A dimensão de representação de uma álgebra de Artin foi introduzida por Auslander em [Aus71]. Ele acreditava que essa invariante poderia dar uma medida de quão longe está uma álgebra de ser de tipo finito. Auslander mostrou que uma álgebra $\Lambda$ não semisimples é de tipo finito se, e somente se, a dimensão de representação rep.dim $\Lambda$ é 2. Iyama [Iya03] provou que a dimensão de representação é sempre finita e Rouquier [Rou06] construiu exemplos de álgebras com rep.dim $\Lambda=r$ para todo $r \geq 1$.

Igusa e Todorov deram, em [IT05], uma conexão interessante com a conjectura finitística. Eles provaram que, se $\Lambda$ tem dimensão de representação no máximo 3 então a dimensão finitística de $\Lambda$ é finita, isto é, o $\max \{\operatorname{dp} M \mid \operatorname{dp} M<\infty\}$ é finito.

A primeira seção trata de resoluções de aproximação, as quais, na Seção 5.2, nos darão um critério para calcular a dimensão de representação de uma álgebra.

Nas seções 5.3 e 5.4 mostramos que, para uma álgebra $\Lambda$, se ind $\Lambda$ possuir uma trisecção $(\mathcal{A}, \mathcal{B}, \mathcal{C})$ com certas propriedades, então é possível calcular a dimensão de representação de $\Lambda$ através das álgebras suporte de $\mathcal{A}$ e $\mathcal{C}$. A partir dos resultados dessas seções conseguimos mostrar, por exemplo, que uma álgebra de tipo ada tem dimensão de representação no máximo 3 (Teorema 5.5.3).

Finalizamos esse capítulo com alguns resultados que relacionam as dimensões de representação das álgebras envolvidas em um produto fibrado orientado.

\subsection{Resolução de aproximação}

A dimensão de representação foi definida por Auslander em [Aus71]. Neste mesmo trabalho ele mostra algumas equivalências para a definição original, dando-nos algumas técnicas para calcular essa invariante. Uma dessas técnicas utiliza as representações de aproximação.

Lembremos primeiramente a definição de aproximação minimal à direita.

Definição 5.1.1 Sejam $M$ e $N$ dois $\Lambda$-módulos. Um morfismo $f: M \rightarrow N$ é dito minimal à direita se para todo $g$ tal que $f g=f$ tem-se que $g$ é um isomorfismo. 
Definição 5.1.2 Seja $\mathcal{X}$ uma subcategoria plena aditiva de $\bmod \Lambda$ e $M$ um $\Lambda$-módulo. Uma $\mathcal{X}$-aproximação à direita de $M$ é um morfimo $f: X \rightarrow M$ com $X$ em $\mathcal{X}$ tal que a sequência de funtores $\left.\left.\operatorname{Hom}_{\Lambda}(-, X)\right|_{\mathcal{X}} \rightarrow \operatorname{Hom}_{\Lambda}(-, M)\right|_{\mathcal{X}} \rightarrow 0$ é exata. Dizemos que $f$ é uma $\mathcal{X}-$ aproximação minimal à direita de $M$ se $f$ é uma $\mathcal{X}$-aproximação à direita que também é um morfismo minimal à direita.

Seja $f: X \rightarrow M$ é uma $\mathcal{X}$-aproximação à direita de $M$. Por [ARS95] (I.2.2), existe uma decomposição $X=X^{\prime} \oplus X^{\prime \prime}$ tal que $\left.f\right|_{X^{\prime}}: X^{\prime} \rightarrow M$ é minimal à direita e $\left.f\right|_{X^{\prime \prime}}=0$. Além disso, $f$ se fatora por $\left.f\right|_{X^{\prime}}$, ou seja, existe $l: X \rightarrow X^{\prime}$ tal que $f=\left.f\right|_{X^{\prime}} \circ l$. Segue que $\left.f\right|_{X^{\prime}}: X^{\prime} \rightarrow M$ é também uma $\mathcal{X}$-aproximação à direita de $M$. Portanto, sempre que existir uma $\mathcal{X}$-aproximação à direita de $M$ então existe uma $\mathcal{X}$-aproximação minimal à direita de $M$.

Definição 5.1.3 Sejam $\Lambda$ uma álgebra de Artin e $\mathcal{X}$ uma subcategoria plena aditiva de $\bmod \Lambda$. Uma $\mathcal{X}$-resolução de aproximação de comprimento $r$ de um módulo $M$ é uma sequência exata $0 \rightarrow X_{r} \rightarrow X_{r-1} \rightarrow \cdots \rightarrow X_{1} \rightarrow M \rightarrow 0$ com cada $X_{i} \in \mathcal{X}$ e tal que a sequência de funtores

$$
\begin{gathered}
\left.\left.0 \rightarrow \operatorname{Hom}_{\Lambda}\left(-, X_{r}\right)\right|_{\mathcal{X}} \rightarrow \operatorname{Hom}_{\Lambda}\left(-, X_{r-1}\right)\right|_{\mathcal{X}} \rightarrow \cdots \\
\left.\left.\cdots \rightarrow \operatorname{Hom}_{\Lambda}\left(-, X_{1}\right)\right|_{\mathcal{X}} \rightarrow \operatorname{Hom}_{\Lambda}(-, M)\right|_{\mathcal{X}} \rightarrow 0
\end{gathered}
$$

é exata.

Note que se $0 \rightarrow X_{r} \stackrel{\varphi_{r}}{\rightarrow} X_{r-1} \rightarrow \cdots \rightarrow X_{2} \stackrel{\varphi_{2}}{\rightarrow} X_{1} \stackrel{\varphi_{F}}{\rightarrow} M \rightarrow 0$ é uma $\mathcal{X}$-resolução de aproximação então $\varphi_{1}$ e cada $\varphi_{i}: X_{i} \rightarrow \operatorname{ker} \varphi_{i-1}$ são $\mathcal{X}$-aproximações à direita. Se cada um desses morfismos é minimal à direita dizemos que é uma $\mathcal{X}$-resolução de aproximação minimal. Veremos que sempre que existir uma $\mathcal{X}$-resolução de aproximação de $M$ com comprimento $r$ então existe uma que é minimal e com comprimento no máximo $r$.

Lema 5.1.1 Seja $\mathcal{X}$ uma subcategoria plena aditiva de $\bmod \Lambda$ e $M$ um $\Lambda$-módulo. Se existir uma $\mathcal{X}$-resolução de aproximação de $M$ com comprimento $r$, então existe uma $\mathcal{X}$-resolução de aproximação minimal que tem comprimento no máximo $r$.

Prova. Seja $0 \rightarrow X_{r} \stackrel{\varphi_{r}}{\rightarrow} X_{r-1} \rightarrow \cdots \rightarrow X_{2} \stackrel{\varphi_{2}}{\rightarrow} X_{1} \stackrel{\varphi_{1}}{\rightarrow} M \rightarrow 0$ uma $\mathcal{X}$-resolução de aproximação. Como $\varphi_{1}$ é uma $\mathcal{X}$-aproximação de $M$ então existe uma decomposição $X=X_{1}^{\prime} \oplus X_{1}^{\prime \prime}$ tal que $\psi_{1}=\left.\varphi_{1}\right|_{X_{1}^{\prime}}$ é uma $\mathcal{X}$-aproximação minimal à direita de $M$ e $\left.\varphi_{1}\right|_{X_{1}^{\prime \prime}}=0$. Vejamos que ker $\psi_{1}$ é um somando direto de $\operatorname{ker} \varphi_{1}$. Considere $\iota_{1}$ e $\pi_{1}$, respectivamente, a inclusão e a projeção naturais tais que $\pi_{1} \iota_{1}=1_{X_{1}^{\prime}}$. Neste caso, temos que $\psi_{1}=\varphi_{1} \iota_{1}$ e portanto $\operatorname{ker} \psi_{1} \subseteq \operatorname{ker} \varphi_{1}$. Além disso, de $\left.\varphi_{1}\right|_{X_{1}^{\prime \prime}}=0$ segue que $\varphi_{1}=\psi_{1} \pi_{1}$. Temos o seguinte diagrama comutativo de linhas exatas 


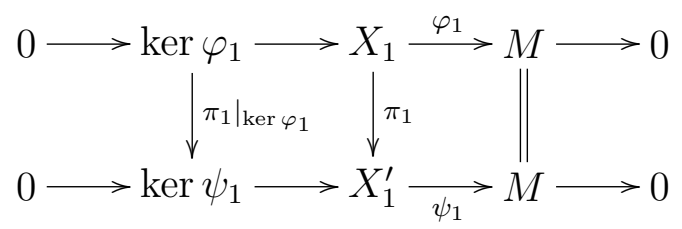

Pelo Lema da Serpente ([Ass97] II.3.6) temos que $\left.\pi_{1}\right|_{\operatorname{ker} \varphi_{1}}$ é um epimorfismo. Além disso, $\left.\left.\pi_{1}\right|_{\operatorname{ker} \varphi_{1}} \circ \iota_{1}\right|_{\operatorname{ker} \psi_{1}}=1_{\operatorname{ker} \psi_{1}}$ e portanto $\left.\pi_{1}\right|_{\operatorname{ker} \varphi_{1}}$ é um epimorfismo que cinde, ou seja, ker $\psi_{1}$ é um somando direto de $\operatorname{ker} \varphi_{1}$.

Não é difícil ver que o morfismo $\left.\pi_{1}\right|_{\operatorname{ker} \varphi_{1}} \circ \varphi_{2}: X_{2} \rightarrow \operatorname{ker} \psi_{1}$ é uma $\mathcal{X}$-aproximação à direita de ker $\psi_{1}$. Novamente, existe uma decomposição $X_{2}=X_{2}^{\prime} \oplus X_{2}^{\prime \prime}$ tal que $\psi_{2}=\left.\varphi_{2}\right|_{X_{2}^{\prime}}$ é uma $\mathcal{X}$-aproximação minimal à direita. Seja $N$ tal que $\operatorname{ker} \psi_{1} \oplus N=\operatorname{ker} \varphi_{1}$ e $\pi_{N}: \operatorname{ker} \varphi_{1} \rightarrow N$ a projeção natural. Então, também temos que $\pi_{N} \circ \varphi_{2}: X_{2} \rightarrow N$ é uma $\mathcal{X}$-aproximação de $N$. Logo, existe um somando $Y$ de $X_{2}$ e um morfismo $f: Y \rightarrow N$ que é uma $\mathcal{X}$-aproximação minimal à direita de $N$. É fácil ver que o morfismo $\theta=\left[\begin{array}{cc}\psi_{2} & 0 \\ 0 & f\end{array}\right]: X_{2}^{\prime} \oplus Y \rightarrow \operatorname{ker} \psi_{1} \oplus N=$ $\operatorname{ker} \varphi_{1}$ é uma $\mathcal{X}$-aproximação minimal à direita de $\operatorname{ker} \varphi_{1}$. Portanto, o núcleo de $\theta$ é um somando de $\operatorname{ker} \varphi_{2}$ e, consequentemente, $\operatorname{ker} \psi_{2}$ também é um somando direto de $\operatorname{ker} \varphi_{2}$.

Repetindo o argumento $t$ vezes teremos a seguinte sequência exata $0 \rightarrow \operatorname{ker} \psi_{t} \rightarrow$ $X_{t}^{\prime} \stackrel{\psi_{t}}{\rightarrow} X_{t-1}^{\prime} \rightarrow \cdots \rightarrow X_{2}^{\prime} \stackrel{\psi_{2}}{\rightarrow} X_{1}^{\prime} \stackrel{\psi_{1}}{\rightarrow} M \rightarrow 0$ onde cada $X_{i}^{\prime}$ é um somando de $X_{i}$, cada $\psi_{i}: X_{i}^{\prime} \rightarrow \operatorname{ker} \psi_{i-1}$ é uma $\mathcal{X}$-aproximação minimal à direita e ker $\psi_{t}$ é um somando direto de ker $\varphi_{t}$. Esse processo termina em no máximo $r$ passos e nos dá uma $\mathcal{X}$-resolução de aproximação minimal de comprimento no máximo $r$.

Dizemos que um $\Lambda$-módulo $\bar{M}$ possui a propriedade de $r$-aproximação em ind $\Lambda$ se cada indecomponível tem uma add $\bar{M}$-resolução de aproximação de comprimento no máximo $r$. Observe que os módulos de $\operatorname{add} \bar{M}$ sempre têm uma add $\bar{M}$-resolução de aproximação de comprimento 1 . Observe também que se $\bar{M}$ tem a propriedade de $r$-resolução então $\bar{M}$ tem a propriedade de $s$-resolução para todo $s \geq r$.

Lema 5.1.2 Sejam $M$ e $\bar{M}$ dois $\Lambda$-módulos. São equivalentes,

(a) A sequência de funtores

$$
\begin{gathered}
\left.\left.0 \rightarrow \operatorname{Hom}_{\Lambda}\left(-, X_{r}\right)\right|_{\text {add } \bar{M}} \rightarrow \operatorname{Hom}_{\Lambda}\left(-, X_{r-1}\right)\right|_{\text {add } \bar{M}} \rightarrow \cdots \\
\left.\left.\quad \cdots \rightarrow \operatorname{Hom}_{\Lambda}\left(-, X_{1}\right)\right|_{\text {add } \bar{M}} \rightarrow \operatorname{Hom}_{\Lambda}(-, M)\right|_{\text {add } \bar{M}} \rightarrow 0
\end{gathered}
$$

é exata.

(b) A sequência

$$
0 \rightarrow \operatorname{Hom}_{\Lambda}\left(N, X_{r}\right) \rightarrow \operatorname{Hom}_{\Lambda}\left(N, X_{r-1}\right) \rightarrow \cdots \rightarrow \operatorname{Hom}_{\Lambda}\left(N, X_{1}\right) \rightarrow \operatorname{Hom}_{\Lambda}(N, M) \rightarrow 0
$$


é exata para cada somando $N$ de $\bar{M}$.

(c) A sequência

$$
\begin{aligned}
& 0 \rightarrow \operatorname{Hom}_{\Lambda}\left(\bar{M}, X_{r}\right) \rightarrow \operatorname{Hom}_{\Lambda}\left(\bar{M}, X_{r-1}\right) \rightarrow \cdots \rightarrow \operatorname{Hom}_{\Lambda}\left(\bar{M}, X_{1}\right) \rightarrow \operatorname{Hom}_{\Lambda}(\bar{M}, M) \rightarrow 0 \\
& \text { é exata. }
\end{aligned}
$$

\subsection{Dimensão de representação}

Lembremos que um $\Lambda$-módulo é chamado de gerador de $\bmod \Lambda$ se todos os módulos projetivos estão em add $M$. Dualmente, $M$ é dito cogerador de $\bmod \Lambda$ se todos os módulos injetivos estão em add $M$. Finalmente, $M$ é dito gerador-cogerador de $\bmod \Lambda$ se for ambos gerador e cogerador $\operatorname{de} \bmod \Lambda$.

Definição 5.2.1 Seja $\Lambda$ uma álgebra de Artin não semi-simples. A dimensão de representação rep.dim $\Lambda$ é o ínfimo das dimensões globais das álgebras $\operatorname{End} M$ onde $M$ é um gerador-cogerador de $\bmod \Lambda$.

Uma técnica utilizada para se calcular a dimensão de representação de uma álgebra é verificar a existência de um gerador-cogerador $\operatorname{de} \bmod \Lambda$ que possui a propriedade de $r$ aproximação em ind $\Lambda$.

A demonstração do resultado a seguir pode ser encontrada por exemplo em [CP04, EHIS04, Xi02].

Teorema 5.2.1 Para uma álgebra de Artin $\Lambda$, a dimensão de representação é no máximo $r+1$ se, e somente se, existe um gerador-cogerador de $\bmod \Lambda$ que possui a propriedade de $r$-aproximação em ind $\Lambda$.

Um módulo $\bar{M}$ é dito um gerador de Auslander se for um gerador-cogerador de $\bmod \Lambda$ que possui a propriedade de $r$-aproximação em ind $\Lambda$ onde $r+1$ é justamente rep.dim $\Lambda$.

Com o resultado acima é possível mostrar que classes importantes de álgebras tem dimensão de representação no máximo três, como por exemplo, as álgebras inclinadas e as álgebras laura [APT06] e as extensões de álgebras hereditárias [CP04].

Para exemplificar veremos que para uma álgebra hereditária $\Lambda$, o módulo $\bar{M}=\Lambda \oplus D \Lambda$ é um gerador-cogerador de $\bmod \Lambda$ que possui a propriedade de 2 -aproximação em ind $\Lambda$ e portanto rep.dim $\Lambda \leq 3$. Tal observação é também devida a Auslander ([Aus71]). 
Exemplo 5.2.2 Seja $\Lambda$ uma álgebra hereditária não semisimples. O módulo $\bar{M}=\Lambda \oplus D \Lambda$ é de fato um gerador-cogerador de $\bmod \Lambda$. Vejamos que ele possui a propriedades de 2aproximação em $\operatorname{ind} \Lambda$. Seja $M$ um $\Lambda$-módulo indecomponível. Se $M$ está em add $\bar{M}$ então a sequência exata $0 \rightarrow M \rightarrow M \rightarrow 0$ é trivialmente uma add $\bar{M}$-resolução de aproximação de comprimento 1 para $M$. Consideremos então o caso em que $M$ não está em add $\bar{M}$. Nesse caso, como $\Lambda$ é hereditária temos que $\mathrm{dp} M=1$. Consideremos a resolução projetiva de $M$ : $0 \rightarrow P_{1} \rightarrow P_{0} \rightarrow M \rightarrow 0$. Se $P$ é um $\Lambda$-módulo projetivo então

$$
0 \rightarrow \operatorname{Hom}_{\Lambda}\left(P, P_{1}\right) \rightarrow \operatorname{Hom}_{\Lambda}\left(P, P_{0}\right) \rightarrow \operatorname{Hom}_{\Lambda}(P, M) \rightarrow 0
$$

é exata. Se I é um injetivo então $\operatorname{Hom}_{\Lambda}(I, M)=0$ pois caso contrário, como $\Lambda$ é hereditária teríamos $M$ injetivo, uma contradição. Portanto,

$$
\left.\left.\left.0 \rightarrow \operatorname{Hom}_{\Lambda}\left(-, P_{1}\right)\right|_{\operatorname{add} \bar{M}} \rightarrow \operatorname{Hom}_{\Lambda}\left(-, P_{0}\right)\right|_{\operatorname{add} \bar{M}} \rightarrow \operatorname{Hom}_{\Lambda}(-, M)\right|_{\operatorname{add} \bar{M}} \rightarrow 0
$$

é exata.

Para uma álgebra $\Lambda$ não semisimples de tipo finito podemos considerar $\bar{M}$ a soma de todos os módulos indecomponíveis, pois ind $\Lambda$ é finita. É fácil ver que $\bar{M}$ é um gerador-cogerador e que possui a propriedade de 1 -aproximação em $\operatorname{ind} \Lambda$. Portanto, rep.dim $\Lambda=2$.

Consequentemente, para uma álgebra $\Lambda$ hereditária, segue que

- $\operatorname{rep} \operatorname{dim} \Lambda=2$ se, e somente se, $\Lambda$ é de tipo finito e

- $\operatorname{rep} \operatorname{dim} \Lambda=3$ se, e somente se, $\Lambda$ é de tipo infinito.

\subsection{Dimensão de representação e álgebras suporte}

Para uma álgebra de $\operatorname{Artin} \Lambda$, a partir de uma trisecção $(\mathcal{A}, \mathcal{B}, \mathcal{C})$ de $\operatorname{ind} \Lambda$ com algumas propriedades conseguimos relacionar a dimensão de representação de $\Lambda$ com as dimensões de representações das álgebras suporte associadas as subcategoras $\mathcal{A}$ e $\mathcal{C}$. Para nosso primeiro resultado, por analogia ao caso do produto fibrado Nakayama orientado, vamos supor que $\operatorname{ind} \Lambda_{\mathcal{C}}$ é fechada para sucessores ou que ind ${ }_{\mathcal{A}} \Lambda$ é fechada para predecessores. Para ilustrar essas hipóteses faremos um exemplo.

Exemplo 5.3.1 Seja $\Lambda$ a álgebra dada pela aljava

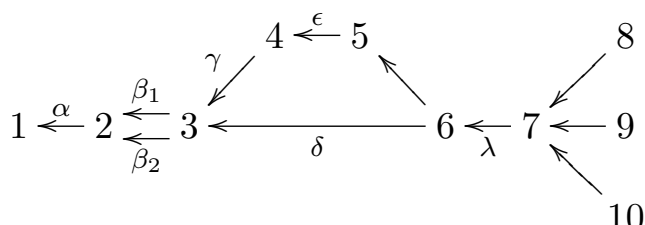

e pelas relações $\beta_{i} \alpha=\gamma \beta_{i}=\delta \beta_{i}=\epsilon \gamma=\lambda \delta=0$. 
Temos que ind ${ }_{\left(\mathcal{L}_{\Lambda}\right)} \Lambda$ consiste de todos os predecessors de $S_{3}$, isto é, $\operatorname{ind}{ }_{\left(\mathcal{L}_{\Lambda}\right)} \Lambda=\operatorname{Pred} S_{3} e$ portanto é fechada para predecessores. Por outro lado, temos que $\Lambda_{\left(\mathcal{R}_{\Lambda}\right)}$ é a álgebra hereditária $4 \leftarrow 5 \leftarrow 6<7 \longleftarrow_{10}^{8}$ e o módulo ${ }_{5}^{6}{ }_{3} \notin$ ind $\Lambda_{\left(\mathcal{R}_{\Lambda}\right)}$ é um sucessor de $S_{5} \in$ ind $\Lambda_{\left(\mathcal{R}_{\Lambda}\right)}$ o que mostra que ind $\Lambda_{\left(\mathcal{R}_{\Lambda}\right)}$ não é fechada para sucessores. Ver esquema da aljava de AuslanderReiten no Apêndice A.

Para uma subcategoria $\mathcal{C}$ de ind $\Lambda$ denotamos seu complementar ind $\Lambda \backslash \mathcal{C}$ por $\mathcal{C}^{c}$

Teorema 5.3.2 Seja $\Lambda$ uma álgebra de Artin $\operatorname{com}(\mathcal{A}, \mathcal{B}, \mathcal{C})$ uma trisecção de ind $\Lambda$. Se

(a) $\left(\mathcal{A} \cup \operatorname{ind} \Lambda_{\mathcal{C}}\right)^{c}$ é finita e ind $\Lambda_{\mathcal{C}}$ é fechada para sucessores

ou

(b) $\left(\operatorname{ind}_{\mathcal{A}} \Lambda \cup \mathcal{C}\right)^{c}$ é finita $e \operatorname{ind}_{\mathcal{A}} \Lambda$ é fechada para predecessores

Então,

$\operatorname{rep} \cdot \operatorname{dim} \Lambda \leq \max \left\{\operatorname{rep} \cdot \operatorname{dim}_{\mathcal{A}} \Lambda, \operatorname{rep} \cdot \operatorname{dim} \Lambda_{\mathcal{C}}\right\}$

Prova. Suponha que rep. $\operatorname{dim}_{\mathcal{A}} \Lambda=r+1$ e que rep.dim $\Lambda_{\mathcal{C}}=s+1$. Seja $\bar{Y}$ um gerador de Auslander de ${ }_{\mathcal{A}} \Lambda$ e seja $\bar{X}$ um gerador de Auslander de $\Lambda_{\mathcal{C}}$. Suponhamos que vale (a) e consideremos os seguintes módulos:

- $\bar{Y}^{\prime}$ a soma direta de todos os somandos indecomponíveis de $\bar{Y}$ que estão em $\mathcal{A}$ mas não estão em $\operatorname{ind} \Lambda_{\mathcal{C}} \mathrm{e}$

- $Z$ a soma direta de todos os módulos indecomponíveis que estão em $\left(\mathcal{A} \cup \operatorname{ind} \Lambda_{\mathcal{C}}\right)^{c}$.

Mostraremos que $\bar{M}=\bar{Y}^{\prime} \oplus Z \oplus \bar{X}$ é um gerador-cogerador de $\bmod \Lambda$ que tem a propriedade de $\max \{r, s\}$-aproximação em ind $\Lambda$.

Seja $P$ um $\Lambda$-módulo projetivo indecomponível. Se $P$ está em $\mathcal{A}$ mas não em ind $\Lambda_{\mathcal{C}}$ então $P$ é um ${ }_{\mathcal{A}} \Lambda$-módulo projetivo e portanto um somando de $\bar{Y}^{\prime}$. Se $P$ está em $\left(\mathcal{A} \cup \operatorname{ind} \Lambda_{\mathcal{C}}\right)^{c}$ então é um somando de $Z$. Se $P$ está em ind $\Lambda_{\mathcal{C}}$ então $P$ é um $\Lambda_{\mathcal{C}}$-módulo projetivo e portanto um somando de $\bar{X}$. Seja $I$ um $\Lambda$-módulo injetivo indecomponível. Se $I$ está em $\mathcal{A}$ mas não em ind $\Lambda_{\mathcal{C}}$ estão $I$ é um ${ }_{\mathcal{A}} \Lambda$-módulo injetivo e então $I$ é um somando de $\bar{Y}^{\prime}$. Se $I$ está em $\left(\mathcal{A} \cup \operatorname{ind} \Lambda_{\mathcal{C}}\right)^{c}$ então $I$ é um somando de $Z$. Se $I$ está em ind $\Lambda_{\mathcal{C}}$ então $I$ é um $\Lambda_{\mathcal{C}}$-módulo injetivo e portanto um somando de $\bar{X}$. Portanto $\bar{M}$ é um gerador-cogerador de $\bmod \Lambda$.

Para mostrar que $\bar{M}$ tem a propriedade de $\max \{r, s\}$-aproximação em ind $\Lambda$, consideremos $M$ um $\Lambda$-módulo indecomponível. Se $M$ está em add $\bar{M}$ não há nada a fazer. Suponhamos que $M$ não está em $\operatorname{add} \bar{M}$, portanto $M$ está em $\mathcal{A} \cup \operatorname{ind} \Lambda_{\mathcal{C}}$. 
Suponhamos primeiramente que $M$ está em $\mathcal{A}$ mas não em ind $\Lambda_{\mathcal{C}}$. Neste caso, existe uma $\operatorname{add} Y$-resolução de aproximação de comprimento $r \operatorname{em} \bmod _{\mathcal{A}} \Lambda$ :

$$
\text { (1) } 0 \rightarrow Y_{r} \stackrel{\varphi_{r}}{\rightarrow} Y_{r-1} \rightarrow \cdots \rightarrow Y_{2} \stackrel{\varphi_{2}}{\rightarrow} Y_{1} \stackrel{\varphi_{1}}{\rightarrow} M \rightarrow 0
$$

Como $\mathcal{A}$ é fechada para predecessores e ind $\Lambda_{\mathcal{C}}$ é fechada para sucessores então cada $Y_{i}$ está em $\operatorname{add} \bar{Y}^{\prime} \subseteq \operatorname{add} \bar{M}$ e cada $K_{i}=\operatorname{Ker} \varphi_{i}$ está em $\operatorname{add}\left(\mathcal{A} \backslash \operatorname{ind} \Lambda_{\mathcal{C}}\right)$ para cada $i \in\{1, \ldots, r-1\}$. Também, como $\mathcal{A}$ é uma subcategoria plena de ind $\Lambda$, a sequência induzida de funtores

$$
\begin{gathered}
\left.\left.0 \rightarrow \operatorname{Hom}_{\Lambda}\left(-, Y_{r}\right)\right|_{\operatorname{add} \bar{Y}^{\prime}} \rightarrow \operatorname{Hom}_{\Lambda}\left(-, Y_{r-1}\right)\right|_{\operatorname{add} \bar{Y}^{\prime}} \rightarrow \cdots \\
\left.\left.\quad \cdots \rightarrow \operatorname{Hom}_{\Lambda}\left(-, Y_{1}\right)\right|_{\operatorname{add} \bar{Y}^{\prime}} \rightarrow \operatorname{Hom}_{\Lambda}(-, M)\right|_{\text {add } \bar{Y}^{\prime}} \rightarrow 0
\end{gathered}
$$

é exata.

Para cada somando indecomponível $L$ de $\bar{X}$ temos $\operatorname{Hom}_{\Lambda}(L, M)=0$ e $\operatorname{Hom}_{\Lambda}\left(L, K_{i}\right)=0$ para $i \in\{1, \ldots, r-1\}$, pois $L \in \operatorname{ind} \Lambda_{\mathcal{C}}$ e ind $\Lambda_{\mathcal{C}}$ é fechada para sucessores. Então

$$
\begin{gathered}
\left.\left.0 \rightarrow \operatorname{Hom}_{\Lambda}\left(-, Y_{r}\right)\right|_{\text {add } \bar{X}} \rightarrow \operatorname{Hom}_{\Lambda}\left(-, Y_{r-1}\right)\right|_{\operatorname{add} \bar{X}} \rightarrow \cdots \\
\left.\left.\quad \cdots \rightarrow \operatorname{Hom}_{\Lambda}\left(-, Y_{1}\right)\right|_{\text {add } \bar{X}} \rightarrow \operatorname{Hom}_{\Lambda}(-, M)\right|_{\text {add } \bar{X}} \rightarrow 0
\end{gathered}
$$

é exata. E, como $\mathcal{A}$ é fechada para predecessores, $\operatorname{temos} \operatorname{Hom}_{\Lambda}(Z, M)=0$ e $\operatorname{Hom}_{\Lambda}\left(Z, K_{i}\right)=0$, logo a sequência

$$
\begin{gathered}
\left.\left.0 \rightarrow \operatorname{Hom}_{\Lambda}\left(-, Y_{r}\right)\right|_{\text {add } Z} \rightarrow \operatorname{Hom}_{\Lambda}\left(-, Y_{r-1}\right)\right|_{\text {add } Z} \rightarrow \cdots \\
\left.\left.\quad \cdots \rightarrow \operatorname{Hom}_{\Lambda}\left(-, Y_{1}\right)\right|_{\operatorname{add} Z} \rightarrow \operatorname{Hom}_{\Lambda}(-, M)\right|_{\text {add } Z} \rightarrow 0
\end{gathered}
$$

também é exata. Portanto (1) é uma add $\bar{M}$-resolução de aproximação de $M$ com comprimento $r$.

Suponhamos agora que $M$ está em ind $\Lambda_{\mathcal{C}}$. Neste caso, existe uma add $\bar{X}$-resolução de aproximação de comprimento $s$ :

$$
\text { (2) } 0 \rightarrow X_{s} \stackrel{\psi_{s}}{\rightarrow} X_{s-1} \rightarrow \cdots \rightarrow X_{2} \stackrel{\psi_{2}}{\rightarrow} X_{1} \stackrel{\psi_{1}}{\rightarrow} M \rightarrow 0 \text {. }
$$

Como ind $\Lambda_{\mathcal{C}}$ é plena em ind $\Lambda$, a sequência induzida de funtores

$$
\begin{gathered}
\left.\left.0 \rightarrow \operatorname{Hom}_{\Lambda}\left(-, X_{s}\right)\right|_{\text {add } \bar{X}} \rightarrow \operatorname{Hom}_{\Lambda}\left(-, X_{s-1}\right)\right|_{\text {add } \bar{X}} \rightarrow \cdots \\
\left.\left.\quad \cdots \rightarrow \operatorname{Hom}_{\Lambda}\left(-, X_{1}\right)\right|_{\operatorname{add} \bar{X}} \rightarrow \operatorname{Hom}_{\Lambda}(-, M)\right|_{\text {add } \bar{X}} \rightarrow 0
\end{gathered}
$$

é exata.

Temos que cada $N_{i}=\operatorname{Ker} \psi_{i}$ está em $\bmod \Lambda_{\mathcal{C}}$ para $i \in\{1, \ldots, s-1\}$. Se $N$ é um somando indecomponível não injetivo de $N_{1}$, então $\tau_{\Lambda}^{-1} N \in \operatorname{ind} \Lambda_{\mathcal{C}}$ uma vez que ind $\Lambda_{\mathcal{C}}$ é fechado para 
sucessores, e portanto

$$
\operatorname{Ext}_{\Lambda}^{1}\left(\bar{Y}^{\prime} \oplus Z, N\right) \cong D \underline{\operatorname{Hom}}_{\Lambda}\left(\tau_{\Lambda}^{-1} N, \bar{Y}^{\prime} \oplus Z\right)=0
$$

Daí, a sequência

$$
\begin{gathered}
\left.\left.0 \rightarrow \operatorname{Hom}_{\Lambda}\left(-, N_{1}\right)\right|_{\operatorname{add}\left(\bar{Y}^{\prime} \oplus Z\right)} \rightarrow \operatorname{Hom}_{\Lambda}\left(-, X_{1}\right)\right|_{\operatorname{add}\left(\bar{Y}^{\prime} \oplus Z\right)} \rightarrow \\
\left.\rightarrow \operatorname{Hom}_{\Lambda}(-, M)\right|_{\operatorname{add}\left(\bar{Y}^{\prime} \oplus Z\right)} \rightarrow 0
\end{gathered}
$$

é exata. O mesmo argumento vale substituindo $M$ por $N_{i}$ para cada $i$ em $\{1, \ldots, s-1\}$ e isto mostra que a sequência

$$
\begin{gathered}
\left.\left.0 \rightarrow \operatorname{Hom}_{\Lambda}\left(-, X_{s}\right)\right|_{\operatorname{add}\left(\bar{Y}^{\prime} \oplus Z\right)} \rightarrow \operatorname{Hom}_{\Lambda}\left(-, X_{s-1}\right)\right|_{\operatorname{add}\left(\bar{Y}^{\prime} \oplus Z\right)} \rightarrow \cdots \\
\left.\left.\cdots \rightarrow \operatorname{Hom}_{\Lambda}\left(-, X_{1}\right)\right|_{\operatorname{add}\left(\bar{Y}^{\prime} \oplus Z\right)} \rightarrow \operatorname{Hom}_{\Lambda}(-, M)\right|_{\operatorname{add}\left(\bar{Y}^{\prime} \oplus Z\right)} \rightarrow 0
\end{gathered}
$$

é exata e portanto (2) é uma add $\bar{M}$-resolução de aproximação de $M$. Logo,

$$
\text { rep. } \operatorname{dim} \Lambda \leq \max \{r+1, s+1\}
$$

A prova supondo a hipótese (b) é análoga.

Exemplo 5.3.3 No exemplo 5.3.1 temos que $\left(\mathcal{L}_{\Lambda},\left(\mathcal{L}_{\Lambda} \cup \mathcal{R}_{\Lambda}\right)^{c}, \mathcal{R}_{\Lambda}\right)$ é uma trisecção de ind $\Lambda$ com $\left(\mathcal{L}_{\Lambda} \cup \mathcal{R}_{\Lambda}\right)^{c}$ infinita. Mas (ind $\left.{ }_{\left(\mathcal{L}_{\Lambda}\right)} \Lambda \cup \mathcal{R}_{\Lambda}\right)^{c}$ é finita e ind ${ }_{\left(\mathcal{L}_{\Lambda}\right)} \Lambda=\operatorname{Pred} S_{3}$ é fechada para predecessores. Pelo Teorema 5.3.2 (b) temos que

$$
\text { rep.dim } \Lambda \leq \max \left\{\text { rep.dim }\left(\mathcal{L}_{\Lambda}\right) \Lambda, \operatorname{rep} \cdot \operatorname{dim} \Lambda_{\left(\mathcal{R}_{\Lambda}\right)}\right\}
$$

Agora, como $\Lambda_{\left(\mathcal{R}_{\Lambda}\right)}$ é uma álgebra hereditária, ${ }_{\left(\mathcal{L}_{\Lambda}\right)} \Lambda$ é inclinada e $\Lambda$ é de tipo infinito, temos que rep.dim $\Lambda=3$.

\subsection{Dimensão de representação e partes direita e esquerda}

Antes de enunciar nosso próximo resultado sobre dimensão de representação precisaremos de mais algumas definições e alguns resultados.

Seja $\mathcal{X}$ uma subcategoria plena de $\bmod \Lambda$. Dizemos que $\mathcal{X}$ é contravariantemente finita se para cada $\Lambda$-módulo $M$ existir um morfismo $f_{M}: X_{M} \rightarrow M$ com $X_{M}$ em $\mathcal{X}$ tal que todo morfismo $f: X \rightarrow M$ se fatora através de $f_{M}$. Dualmente, define-se subcategoria covariantemente finita. Dizemos que $\mathcal{X}$ é funtorialmente finita se for contravariantemente e covariantemente finita. Finalmente, dizemos que $\mathcal{X}$ é homologicamente finita se for contravariantemente ou covariantemente finita. 
Por exemplo, toda subcategoria finita e toda subcategoria cofinita de $\bmod \Lambda$ são funtorialmente finitas (ver [AS80]).

Se $\mathcal{X}$ é uma subcategoria de $\bmod \Lambda$, aditiva e fechada para extensões, então um módulo $M$ de $\mathcal{X}$ é dito Ext-projetivo em $\mathcal{X}$ se $\left.\operatorname{Ext}_{\Lambda}^{1}(M,-)\right|_{\mathcal{X}}=0$. Dualmente, $M \in \mathcal{X}$ é dito Ext-injetivo em $\mathcal{X}$ se $\left.\operatorname{Ext}_{\Lambda}^{1}(-, M)\right|_{\mathcal{X}}=0$.

Lembremos que um par de subcategorias aditivas $(\mathcal{X}, \mathcal{Y})$ é dito par de torção se não há morfismos de $\mathcal{X}$ para $\mathcal{Y}$ e estas são maximais para essa propriedade, ou seja, se $\operatorname{Hom}_{\Lambda}(M, Y)=0$ para todo $Y$ de $\mathcal{Y}$ então $M$ está em $\mathcal{X}$ e se $\operatorname{Hom}_{\Lambda}(X, M)=0$ para todo $X$ de $\mathcal{X}$ então $M$ está em $\mathcal{Y}$. Um par de torção é dito cindido se $\mathcal{X} \cup \mathcal{Y}$ coincide com $\bmod \Lambda$. Se $(\mathcal{X}, \mathcal{Y})$ é um par de torção então, por [AS81], $M \in \mathcal{X}$ é Ext-projetivo em $\mathcal{X}$ se, e somente se, $\tau_{\Lambda} M$ está em $\mathcal{Y}$ e $N \in \mathcal{Y}$ é Ext-injetivo em $\mathcal{Y}$ se, e somente se, $\tau_{\Lambda}^{-1} N$ está em $\mathcal{X}$. Se $\mathcal{A}$ é uma subcategoria plena de ind $\Lambda$ fechada para predecessores então seu complemento $\mathcal{A}^{c}=\operatorname{ind} \Lambda \backslash \mathcal{A}$ é fechada para sucessores e neste caso $\left(\operatorname{add} \mathcal{A}^{c}, \operatorname{add} \mathcal{A}\right)$ é um par de torção cindido. Denotemos por $E$ a soma direta de um conjunto completo de representantes dos módulos indecomponíveis Ext-injetivos em add $\mathcal{A}$ e por $F$ a soma direta de um conjunto completo de representantes dos módulos indecomponíveis Ext-projetivos em add $\mathcal{A}^{c}$. O lema a seguir é devido a Smalø.

Lema 5.4.1 ([Sma84]) Seja $\mathcal{A}$ uma subcategoria plena de ind $\Lambda$ fechada para predecessores. As seguintes condições são equivalentes:

(a) $\operatorname{add} \mathcal{A}$ é contravariantemente finita;

(b) $\operatorname{add} \mathcal{A}=\operatorname{Cogen} N$ para algum $N \in \bmod \Lambda$;

(c) $\operatorname{add} \mathcal{A}=\operatorname{Cogen} E ;$

(d) $\operatorname{add} \mathcal{A}^{c}$ é covariantemente finita;

(e) $\operatorname{add} \mathcal{A}^{c}=\operatorname{Gen} M$ para algum $M \in \bmod \Lambda$;

(f) $\operatorname{add} \mathcal{A}^{c}=\operatorname{Gen} F$.

Lema 5.4.2 Seja $(\mathcal{A}, \mathcal{B}, \mathcal{C})$ uma trisecção de ind $\Lambda$ tal que $\mathcal{B}$ é finita. Então, $\operatorname{add} \mathcal{C}$ é covariantemente finita se, e somente se, add $\mathcal{A}$ é contravariantemente finita.

Prova.

Como $(\mathcal{A}, \mathcal{B}, \mathcal{C})$ é uma triseç̧ão de ind $\Lambda$ temos que $\mathcal{A}$ e $\mathcal{A} \cup \mathcal{B}$ são fechadas para predecessores e $\mathcal{C}$ e $\mathcal{B} \cup \mathcal{C}$ são fechadas para sucessores. Suponha que add $\mathcal{A}$ é contravariantemente finita, então pelo lema anterior $\operatorname{add} \mathcal{A}=\operatorname{Cogen} N$ para $\operatorname{algum} N \in \bmod \Lambda$. Seja $X$ a soma direta dos módulos indecomponíveis que estão em $\mathcal{B}$. $\operatorname{Então,~} \operatorname{add}(\mathcal{A} \cup \mathcal{B}) \subseteq \operatorname{Cogen}(N \oplus X)$ e 
$\operatorname{Cogen}(N \oplus X) \subseteq \operatorname{add}(\mathcal{A} \cup \mathcal{B})$ pois $\mathcal{A} \cup \mathcal{B}$ é fechada para predecessores. Portanto, $\operatorname{add}(\mathcal{A} \cup \mathcal{B})$ é também contravarientemente finita e finalmente segue que add $\mathcal{C}$ é covariantemente finita.

Lema 5.4.3 ([APT06](1.4)) Seja $M$ um $\Lambda$-módulo. Se X está em Gen $M$ então existe um epimorfismo $f: M_{1} \rightarrow X$ com $M_{1}$ em add $M$ tal que $\operatorname{Hom}\left(-, M_{1}\right) \rightarrow \operatorname{Hom}(-, X) \rightarrow 0$ é uma cobertura projetiva.

Lema 5.4.4 ([APT06](2.2)(f)) Sejam $\Lambda$ uma álgebra inclinada e $M=T \oplus D A$ um $\Lambda$ módulo com $T$ inclinante convexo. Dado $X$ em $\operatorname{Gen} M$, se $f: M_{1} \rightarrow X$ com $M_{1}$ em add $M$ é um epimorfismo tal que $\operatorname{Hom}\left(-, M_{1}\right) \rightarrow \operatorname{Hom}(-, X) \rightarrow 0$ é uma cobertura projetiva, então ker $f$ está em add $T$.

Seja, agora, $\mathcal{C}$ uma subcategoria de ind $\Lambda$ fechada para sucessores tal que add $\mathcal{C}$ é covariantemente finita. Denotemos por $F$ a soma direta de todos os módulos indecomponíveis Ext-projetivos em add $\mathcal{C}$ e por $N$ a soma direta de todos os módulos indecomponíveis injetivos que estão em $\mathcal{C}$.

Lema 5.4.5 ([ACT09] (5.3)) Seja C uma subcategoria plena de ind $\Lambda$ fechada para sucessores tal que addC é covariantemente finita. Então,

(a) F é convexo se, e somente se, $\mathcal{C} \subseteq \mathcal{R}_{\Lambda}$.

(b) Se addC contém todos os $\Lambda$-módulos injetivos então $\mathcal{C} \subseteq \mathcal{R}_{\Lambda}$ se, e somente se, $\Lambda$ é uma álgebra inclinada tendo $F$ como uma fatia completa.

Ainda denotando $F$ e $N$ como acima, ou seja, por $F$ a soma direta de todos os módulos indecomponíveis Ext-projetivos em addC e por $N$ a soma direta de todos os módulos indecomponíveis injetivos que estão em $\mathcal{C}$, temos o seguinte resultado.

Lema 5.4.6 Seja $\mathcal{C} \subseteq \mathcal{R}_{\Lambda}$ uma subcategoria de ind $\Lambda$ fechada para sucessores tal que addC é covariantemente finita. Para cada $M$ em $\mathcal{C}$ existe uma sequência exata

$$
0 \rightarrow F_{2} \rightarrow F_{1} \oplus I_{1} \rightarrow M \rightarrow 0
$$

com $F_{1}, F_{2} \in \operatorname{add} F$ e $I_{1} \in \operatorname{add} N$, que é uma $\operatorname{add}(F \oplus N)$-resolução de aproximação de comprimento 2 de $M$.

Prova. Como $\mathcal{C} \subseteq \mathcal{R}_{\Lambda}$, segue do Lema 4.1.1 (c') que $\mathcal{C} \subseteq \mathcal{R}_{\left(\Lambda_{\mathcal{C}}\right)}$. E como add $\mathcal{C}$ contém todos os $\Lambda_{\mathcal{C}}$-módulos injetivos segue do Lema 5.4.5 que $\Lambda_{\mathcal{C}}$ é uma álgebra inclinada e que $F$ é um $\Lambda_{\mathcal{C}}$-módulo inclinante convexo. 
Como add $\mathcal{C}$ é covariantemente finita temos, pelo Lema 5.4.1, que addC $=$ Gen $F$ e daí $\operatorname{add} \mathcal{C} \subseteq \operatorname{Gen}(F \oplus N)$. Então, pelo Lema 5.4.3, para todo módulo $M \in \mathcal{C}$ existe uma sequência exata $(*) \quad 0 \rightarrow K \rightarrow F_{1} \oplus I_{1} \rightarrow M \rightarrow 0 \operatorname{com} F_{1}$ em $\operatorname{add} F, I_{1}$ em $\operatorname{add} N$ tal que a sequência curta de funtores

$$
\left.\left.\left.0 \rightarrow \operatorname{Hom}_{\Lambda}(-, K)\right|_{\operatorname{add}(F \oplus N)} \rightarrow \operatorname{Hom}_{\Lambda}\left(-, F_{1} \oplus I_{1}\right)\right|_{\operatorname{add}(F \oplus N)} \rightarrow \operatorname{Hom}_{\Lambda}(-, M)\right|_{\operatorname{add}(F \oplus N)} \rightarrow 0
$$

é exata. Agora, pelo Lema 5.4.4, temos que $K$ está em add $F$ e portanto $(*)$ é uma add $(F \oplus N)$ resolução de aproximação de comprimento 2 de $M$.

Podemos relacionar a dimensão de representação da álgebra $\Lambda$ com as dimensões de representação das álgebras suporte de $\mathcal{A}$ e de $\mathcal{C}$ quando $(\mathcal{A}, \mathcal{B}, \mathcal{C})$ é uma trisecção de ind $\Lambda$ com certas propriedades.

Teorema 5.4.7 Seja $\Lambda$ uma álgebra de Artin de tipo infinito e $(\mathcal{A}, \mathcal{B}, \mathcal{C})$ uma trisecção de $\operatorname{ind} \Lambda \operatorname{com} \mathcal{B}$ finita.

(a) $S e \mathcal{C} \subseteq \mathcal{R}_{\Lambda}$ e addC é covariantemente finita então

$$
\text { rep.dim } \Lambda=\max \left\{3, \operatorname{rep} \cdot \operatorname{dim}_{\mathcal{A}} \Lambda\right\}
$$

(b) $S e \mathcal{A} \subseteq \mathcal{L}_{\Lambda}$ e add $\mathcal{A}$ é contravariantemente finita então

$$
\text { rep.dim } \Lambda=\max \left\{3, \text { rep. } \operatorname{dim} \Lambda_{\mathcal{C}}\right\}
$$

Faremos a demonstração do teorema acima em partes. Começaremos mostrando uma das desigualdades.

Lema 5.4.8 Sejam $\Lambda$ uma álgebra de Artin e $(\mathcal{A}, \mathcal{B}, \mathcal{C})$ uma trisecção de ind $\Lambda$ com $\mathcal{B}$ finita,

(a) $S e \mathcal{C} \subseteq \mathcal{R}_{\Lambda} e$ addC é covariantemente finita então

$$
\text { rep.dim } \Lambda \leq \max \left\{3, \operatorname{rep} \cdot \operatorname{dim}_{\mathcal{A}} \Lambda\right\}
$$

(b) $S e \mathcal{A} \subseteq \mathcal{L}_{\Lambda}$ e add $\mathcal{A}$ é contravariantemente finita então

$$
\text { rep.dim } \Lambda \leq \max \left\{3, \text { rep. } \operatorname{dim} \Lambda_{\mathcal{C}}\right\}
$$

Prova. Faremos a prova de (a) pois (b) é dual. Suponhamos que rep.dim ${ }_{\mathcal{A}} \Lambda=r+1$ e seja $\bar{X}$ um gerador de Auslander de ${ }_{\mathcal{A}} \Lambda$. Consideremos os seguintes módulos:

- $\bar{X}^{\prime}$ a soma direta de todos os somandos indecomponíveis de $\bar{X}$ que estão em $\mathcal{A}$; 
- $Z$ a soma direta de todos os $\Lambda$-modulos indecomponíveis que estão em $\mathcal{B}$;

- F a soma direta de todos os indecomponíveis Ext-projetivos em addC ; e

- $N$ a soma direta de todos os $\Lambda$-modulos injetivos indecomponíveis que estão em $\mathcal{C}$.

Provaremos que $\bar{M}=\bar{X}^{\prime} \oplus Z \oplus F \oplus N$ é um gerador-cogerador de $\bmod \Lambda$ e que ele tem a propriedade de $\max \{2, r\}$-aproximação em ind $\Lambda$.

Seja $P$ um $\Lambda$-módulo projetivo indecomponível. Se $P$ está em $\mathcal{A}$ então $P$ é um ${ }_{\mathcal{A}} \Lambda$-módulo projetivo e portanto é um somando de $\bar{X}^{\prime}$. Se $P$ está em $\mathcal{B}$ então é um somando de $Z$. Se $P$ está em $\mathcal{C}$ então $P$ é um Ext-projetivo em addC e portanto um somando de $F$. Logo $M$ é um gerador de $\bmod \Lambda$.

Seja $I$ um $\Lambda$-módulo injetivo indecomponível. Se $I$ está em $\mathcal{A}$ então $I$ é um ${ }_{\mathcal{A}} \Lambda$-módulo injetivo e portanto um somando de $\bar{X}^{\prime}$. Se $I$ está em $\mathcal{B}$ então $I$ é somando de $Z$. Se $I$ está em $\mathcal{C}$ então $I$ é um somando de $N$. Logo $\bar{M}$ é também cogerador de $\bmod \Lambda$.

Para mostrar que $\bar{M}$ tem a propriedade de $\max \{2, r\}$-resolução de aproximação em ind $\Lambda$ consideremos $M$ um $\Lambda$-módulo indecomponível. Se $M$ está em add $\bar{M}$ não há nada a fazer, então suponhamos que $M \notin \operatorname{add} \bar{M}$. Neste caso, temos $M \in \mathcal{A} \cup \mathcal{C}$. Consideremos agora dois casos, primeiramente quando $M$ está em $\mathcal{A}$ e em seguida quando $M$ está $\mathcal{C}$.

Se $M$ está em $\mathcal{A}$ então $M$ é um ${ }_{\mathcal{A}} \Lambda$-módulo e portanto existe uma $\bar{X}$-resolução de aproximação de comprimento $r$, ou seja, uma sequência exata

$$
\text { (1) } 0 \rightarrow X_{r} \stackrel{\varphi_{r}}{\rightarrow} X_{r-1} \rightarrow \cdots \rightarrow X_{2} \stackrel{\varphi_{2}}{\rightarrow} X_{1} \stackrel{\varphi_{1}}{\rightarrow} M \rightarrow 0
$$

com $X_{i}$ em add $\bar{X}$ tal que a sequência de funtores

$$
\begin{gathered}
\left.\left.0 \rightarrow \operatorname{Hom}_{\mathcal{A} \Lambda}\left(-, X_{r}\right)\right|_{\operatorname{add} \bar{X}} \rightarrow \operatorname{Hom}_{\mathcal{A} \Lambda}\left(-, X_{r-1}\right)\right|_{\operatorname{add} \bar{X}} \rightarrow \cdots \\
\left.\left.\quad \cdots \rightarrow \operatorname{Hom}_{\mathcal{A} \Lambda}\left(-, X_{1}\right)\right|_{\operatorname{add} \bar{X}} \rightarrow \operatorname{Hom}_{\mathcal{A} \Lambda}(-, M)\right|_{\operatorname{add} \bar{X}} \rightarrow 0
\end{gathered}
$$

é exata.

Como $\mathcal{A}$ é fechada para predecessores então cada $X_{i}$ está em add $\mathcal{A}$ e portanto cada $X_{i}$ está em $\operatorname{add} \bar{X}^{\prime} \subseteq \operatorname{add} \bar{M}$. Como $\mathcal{A}$ é uma subcategoria plena de ind $\Lambda$ e $\operatorname{add} \bar{X}^{\prime} \subseteq \operatorname{add} \bar{X}$ então a sequencia de funtores induzida

$$
\begin{gathered}
\left.\left.0 \rightarrow \operatorname{Hom}_{\Lambda}\left(-, X_{r}\right)\right|_{\text {add } \bar{X}^{\prime}} \rightarrow \operatorname{Hom}_{\Lambda}\left(-, X_{r-1}\right)\right|_{\text {add } \bar{X}^{\prime}} \rightarrow \cdots \\
\left.\left.\cdots \rightarrow \operatorname{Hom}_{\Lambda}\left(-, X_{1}\right)\right|_{\text {add } \bar{X}^{\prime}} \rightarrow \operatorname{Hom}_{\Lambda}(-, M)\right|_{\text {add } \bar{X}^{\prime}} \rightarrow 0
\end{gathered}
$$

é exata.

Além disso, como cada somando indecomponível de $Z \oplus F \oplus N$ está em $\mathcal{B} \cup \mathcal{C}$ e como $\mathcal{A}$ é fechada para predecessores então $\operatorname{Hom}_{\Lambda}(Z \oplus F \oplus N, M)=0$ e denotando por $K_{i}$ o núcleo de cada $\varphi_{i}$ também temos que $\operatorname{Hom}_{\Lambda}\left(Z \oplus F \oplus N, K_{i}\right)=0$ para cada $i \in\{1, \ldots, r-1\}$. Portanto, 
a sequência

$$
\begin{gathered}
\left.\left.0 \rightarrow \operatorname{Hom}_{\Lambda}\left(-, X_{r}\right)\right|_{\operatorname{add}(Z \oplus F \oplus N)} \rightarrow \operatorname{Hom}_{\Lambda}\left(-, X_{r-1}\right)\right|_{\operatorname{add}(Z \oplus F \oplus N)} \rightarrow \cdots \\
\left.\left.\quad \cdots \rightarrow \operatorname{Hom}_{\Lambda}\left(-, X_{1}\right)\right|_{\operatorname{add}(Z \oplus F \oplus N)} \rightarrow \operatorname{Hom}_{\Lambda}(-, M)\right|_{\operatorname{add}(Z \oplus F \oplus N)} \rightarrow 0
\end{gathered}
$$

é também exata, o que mostra que (1) é uma add $\bar{M}$-resolução de aproximação de comprimento $r$ de $M$.

Vejamos agora o que acontece quando $M$ está em $\mathcal{C}$. Pelo Lema 5.4.6, existe uma add $(F \oplus$ $N$ )-resolução de aproximação de comprimento 2 de $M$ :

$$
\text { (2) } \quad 0 \rightarrow F_{2} \rightarrow F_{1} \oplus I_{1} \rightarrow M \rightarrow 0
$$

$\operatorname{com} F_{1}, F_{2} \in \operatorname{add} F \subseteq \operatorname{add} \bar{M}$ e $I_{1} \in \operatorname{add} N \subseteq \operatorname{add} \bar{M}$

Seja $L$ um somando indecomponível de $\bar{X}^{\prime} \oplus Z$. Se $L$ é um $\Lambda$-módulo projetivo então a sequência $0 \rightarrow \operatorname{Hom}_{\Lambda}\left(L, F_{2}\right) \rightarrow \operatorname{Hom}_{\Lambda}\left(L, F_{1} \oplus I_{1}\right) \rightarrow \operatorname{Hom}_{\Lambda}(L, M) \rightarrow 0$ é exata. Se $L$ não é projetivo, então $\tau L$ não está em $\mathcal{C}$ pois $\mathcal{C}$ é fechada para sucessores e $L$ não está em $\mathcal{C}$. Daí, como $F_{2} \in \operatorname{add} \mathcal{C}$, nós temos que

$$
\operatorname{Ext}_{\Lambda}^{1}\left(L, F_{2}\right) \cong D \overline{\operatorname{Hom}}_{\Lambda}\left(F_{2}, \tau L\right)=0
$$

Portanto, a sequência

$$
\left.\left.\left.0 \rightarrow \operatorname{Hom}_{\Lambda}\left(-, F_{2}\right)\right|_{\operatorname{add}\left(\bar{X}^{\prime} \oplus Z\right)} \rightarrow \operatorname{Hom}_{\Lambda}\left(-, F_{1} \oplus I_{1}\right)\right|_{\operatorname{add}\left(\bar{X}^{\prime} \oplus Z\right)} \rightarrow \operatorname{Hom}_{\Lambda}(-, M)\right|_{\operatorname{add}\left(\bar{X}^{\prime} \oplus Z\right)} \rightarrow 0
$$

é exata e então (2) é uma add $\bar{M}$-resolução de aproximação de $M$ com comprimento 2 . Isto prova que rep.dim $\Lambda \leq \max \{3, r+1\}$ e completa a prova.

Para a segunda desigualdade precisaremos ainda de mais dois lemas.

Lema 5.4.9 Seja $\mathcal{X}$ uma subcategoria aditiva convexa de $\bmod \Lambda$ e $0 \rightarrow X \stackrel{f}{\rightarrow} Y \stackrel{g}{\rightarrow} Z \rightarrow 0$ uma sequência exata em $\mathcal{X}$. Se $K$ é um somando de $X$ que é Ext-injetivo em $\mathcal{X}$ então $K$ é também um somando de $Y$.

Prova. Seja $p: X \rightarrow K$ e $i: K \rightarrow X$ os morfismos naturais tais que $p \circ i=1_{K}$. Consideremos o seguinte diagrama comutativo com linhas exatas

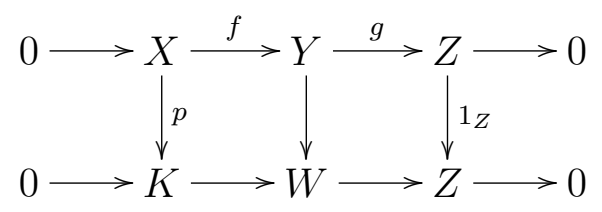

onde $W$ é a soma amalgamada de $f$ e $p$. Pela convexidade de $\mathcal{X}$ temos que $W$ está em $\mathcal{X}$. Como $K$ é Ext-injetivo em $\mathcal{X}$ então a sequência $0 \rightarrow K \rightarrow W \rightarrow Z \rightarrow 0$ cinde e então 
podemos escrever o seguinte diagrama comutativo com linhas exatas

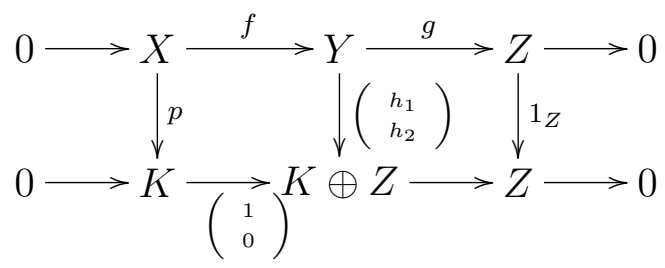

Pela comutatividade do diagrama temos $\left(\begin{array}{c}h_{1} \\ h_{2}\end{array}\right) f=\left(\begin{array}{c}1 \\ 0\end{array}\right) p$, ou seja, $h_{1} \circ f=p$. Portanto, $h_{1} \circ f \circ i=p \circ i=1_{K}$. Isso mostra que $h_{1}: Y \rightarrow K$ é um epimorfismo que cinde e portanto $K$ é um somando de $Y$.

Lema 5.4.10 Seja $\mathcal{X}$ uma subcategoria convexa de $\bmod \Lambda$. Se $f: X \rightarrow M$ é uma $\mathcal{X}$-aproximação minimal à direita de $M$ então o núcleo de $f$ não possui somando direto que é Ext-injetivo em $\mathcal{X}$.

Prova. Suponhamos que $K$ é um somando direto de ker $f$ que é Ext-injetivo em $\mathcal{X}$. Pelo lema anterior temos que $K$ é também somando de $X$. Mas então $f(K)=0$ o que contradiz a minimalidade de $f$ por [ARS95] (I.2.3).

Prova do Teorema 5.4.7.

Provaremos a parte (a) pois (b) é dual.

Pelo Lema 5.4 .8 (a) temos que rep.dim $\Lambda \leq \max \left\{3, \operatorname{rep} \cdot \operatorname{dim}_{\mathcal{A}} \Lambda\right\}$.

Por outro lado, suponhamos que $\operatorname{rep} \operatorname{dim} \Lambda=s+1$. Notemos que $s \geq 2$ pois $\Lambda$ é uma álgebra de tipo infinito. Seja $\bar{M}$ um gerador de Auslander de $\Lambda$. Denotemos $\mathcal{B}^{\prime}=\mathcal{B} \cap \operatorname{ind}_{\mathcal{A}} \Lambda$ e $\mathcal{C}^{\prime}=\mathcal{C} \cap \operatorname{ind}_{\mathcal{A}} \Lambda$, então $\left(\mathcal{A}, \mathcal{B}^{\prime}, \mathcal{C}^{\prime}\right)$ é claramente uma trisecção de ind ${ }_{\mathcal{A}} \Lambda$ com $\mathcal{B}^{\prime}$ uma subcategoria finita. Consideremos os seguintes módulos:

- $\bar{M}^{\prime}$ é a soma direta de todos os somandos indecomponíveis de $\bar{M}$ que estão em $\mathcal{A}$;

- E é a soma direta de todos os indecomponíveis Ext-injetivos em add $\mathcal{A}$ que não são injetivos em $\bmod \Lambda$;

- $Z$ é a soma direta de todos os $\Lambda$-módulos indecomponíveis em $\mathcal{B}^{\prime}$;

- F é a soma direta de todos os indecomponíveis Ext-projetivos em addC'; e

- $N$ é a soma direta de todos os $\Lambda$-módulos injetivos que estão em $\mathcal{C}^{\prime}$.

Mostraremos que o ${ }_{\mathcal{A}} \Lambda$-módulo $\bar{X}=\bar{M}^{\prime} \oplus E \oplus Z \oplus F \oplus N$ é um gerador-cogerador de $\bmod _{\mathcal{A}} \Lambda$ que tem a propriedade de $s$-resolução de aproximação em ind ${ }_{\mathcal{A}} \Lambda$.

Se $P$ é um ${ }_{\mathcal{A}} \Lambda$-módulo projetivo indecomponível então, pelo Lema 4.1.1, $P$ é um $\Lambda$ módulo projetivo que está em $\mathcal{A}$ e então $P$ é um somando de $\bar{M}^{\prime}$. Seja $I$ um ${ }_{\mathcal{A}} \Lambda$-módulo 
injetivo. Se $I$ está em $\mathcal{A}$ então $I$ é um Ext-injetivo em add $\mathcal{A}$ e portanto, se $I$ é injetivo em $\bmod \Lambda$ então $I$ é um somando de $\bar{M}^{\prime}$ ou se $I$ não for injetivo então $I$ é um somando de $E$. Se $I$ está em $\mathcal{B}^{\prime}$ então $I$ é um somando de $Z$ e finalmente se $I$ está em $\mathcal{C}^{\prime}$ então $I$ é um somando

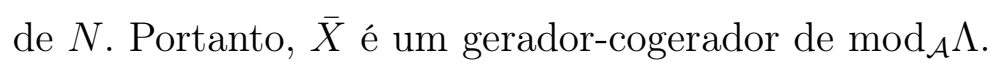

Para mostrar que $\bar{X}$ tem a propriedade de $s$-resolução de aproximação em $\operatorname{ind}_{\mathcal{A}} \Lambda$ consideremos $M$ em $\operatorname{ind}_{\mathcal{A}} \Lambda$ tal que $M$ não está em add $\bar{X}$. Neste caso, $M$ está em $\mathcal{A} \cup \mathcal{C}^{\prime}$.

Pelo Lema 5.4.2, como addC é covariantemente finita em $\bmod \Lambda$, então add $\mathcal{A}$ é contravariantemente finita em $\bmod \Lambda$ e então é também contravariantemente finita em $\bmod _{\mathcal{A}} \Lambda$. Desde que $\left(\mathcal{A}, \mathcal{B}^{\prime}, \mathcal{C}^{\prime}\right)$ é uma trisecção de $\operatorname{ind}_{\mathcal{A}} \Lambda \operatorname{com} \mathcal{B}^{\prime}$ finita então segue que add $\mathcal{C}^{\prime}$ é covariantemente finita em $\bmod _{\mathcal{A}} \Lambda$.

Notemos que $\mathcal{C}^{\prime}$ é fechada para sucessores em $\operatorname{ind}_{\mathcal{A}} \Lambda$ e, pelo Lema 4.1.1, temos que $\mathcal{C}^{\prime} \subseteq \mathcal{R}_{\left({ }_{\mathcal{A}} \Lambda\right)}$. Portanto, se $M$ está em $\mathcal{C}^{\prime}$, pelo Lema 5.4.6, existe uma sequência em $\bmod _{\mathcal{A}} \Lambda$

$$
0 \rightarrow F_{2} \rightarrow F_{1} \oplus I_{1} \rightarrow M \rightarrow 0
$$

$\operatorname{com} F_{1}, F_{2}$ em $\operatorname{add} F \subseteq \operatorname{add} \bar{X}$ e $I_{1}$ em $\operatorname{add} N \subseteq \operatorname{add} \bar{X}$ tal que a sequência

$\left.\left.\left.0 \rightarrow \operatorname{Hom}_{\mathcal{A} \Lambda}\left(-, F_{2}\right)\right|_{\operatorname{add}(F \oplus N)} \rightarrow \operatorname{Hom}_{\mathcal{A} \Lambda}\left(-, F_{1} \oplus I_{1}\right)\right|_{\operatorname{add}(F \oplus N)} \rightarrow \operatorname{Hom}_{\mathcal{A} \Lambda}(-, M)\right|_{\operatorname{add}(F \oplus N)} \rightarrow 0$

é exata.

Seja $L$ um ${ }_{\mathcal{A}} \Lambda$-módulo indecomponível somando de $\bar{M}^{\prime} \oplus Z \oplus E$ então $L$ não está em $\mathcal{C}^{\prime}$. Se $L$ é um ${ }_{\mathcal{A}} \Lambda$-módulo projetivo então

$$
0 \rightarrow \operatorname{Hom}_{\mathcal{A} \Lambda}\left(L, F_{2}\right) \rightarrow \operatorname{Hom}_{\mathcal{A} \Lambda}\left(L, F_{1} \oplus I_{1}\right) \rightarrow \operatorname{Hom}_{\mathcal{A} \Lambda}(L, M) \rightarrow 0
$$

é exata. Se $L$ não é projetivo em $\bmod _{\mathcal{A}} \Lambda$ então $\tau_{\left({ }_{\mathcal{A}} \Lambda\right)} L \notin \mathcal{C}^{\prime}$, pois $\mathcal{C}^{\prime}$ é fechada para sucessores, e como $F_{2}$ está em add $\mathcal{C}^{\prime}$ então

$$
\operatorname{Ext}_{\mathcal{A} \Lambda}^{1}\left(L, F_{2}\right) \cong D \overline{\operatorname{Hom}}_{\mathcal{A} \Lambda}\left(F_{2}, \tau_{\left({ }_{\mathcal{A}} \Lambda\right)} L\right)=0 .
$$

Portanto, a sequência

$$
\begin{gathered}
\left.\left.0 \rightarrow \operatorname{Hom}_{\mathcal{A} \Lambda}\left(-, F_{2}\right)\right|_{\operatorname{add}\left(\bar{M}^{\prime} \oplus Z \oplus E\right)} \rightarrow \operatorname{Hom}_{\mathcal{A} \Lambda}\left(-, F_{1} \oplus I_{1}\right)\right|_{\operatorname{add}\left(\bar{M}^{\prime} \oplus Z \oplus E\right)} \rightarrow \\
\left.\rightarrow \operatorname{Hom}_{\mathcal{A} \Lambda}(-, M)\right|_{\operatorname{add}\left(\bar{M}^{\prime} \oplus Z \oplus E\right)} \rightarrow 0
\end{gathered}
$$

é exata e então $0 \rightarrow F_{2} \rightarrow F_{1} \oplus I_{1} \rightarrow M \rightarrow 0$ é uma add $\bar{X}$-resolução de aproximação de comprimento 2 de $M$.

Vejamos, agora o caso $M \in \mathcal{A}$. Consideremos uma add $\bar{M}$-resolução de aproximação de $M$ de comprimento $s$ :

$$
(*) \quad 0 \rightarrow M_{s} \stackrel{\varphi_{s}}{\rightarrow} M_{s-1} \rightarrow \cdots \rightarrow M_{2} \stackrel{\varphi_{2}}{\rightarrow} M_{1} \stackrel{\varphi_{1}}{\rightarrow} M \rightarrow 0 .
$$


Como $M$ está em $\mathcal{A}$, que é fechada para predecessores, então cada $M_{i}$ está em add $\mathcal{A}$ e, portanto, cada $M_{i}$ está em $\operatorname{add} M^{\prime} \subseteq \operatorname{add} \bar{X}$. Desde que $\operatorname{add} \bar{M}^{\prime} \subseteq \operatorname{add} \bar{M}$ e $\operatorname{ind}_{\mathcal{A}} \Lambda$ é uma subcategoria plena de ind $\Lambda$ então a sequência induzida de funtores

$$
\begin{gathered}
\left.\left.0 \rightarrow \operatorname{Hom}_{\mathcal{A} \Lambda}\left(-, M_{s}\right)\right|_{\mathrm{add} \bar{M}^{\prime}} \rightarrow \operatorname{Hom}_{\mathcal{A} \Lambda}\left(-, M_{s-1}\right)\right|_{\mathrm{add} \bar{M}^{\prime}} \rightarrow \cdots \\
\left.\left.\quad \cdots \rightarrow \operatorname{Hom}_{\mathcal{A} \Lambda}\left(-, M_{1}\right)\right|_{\mathrm{add} \bar{M}^{\prime}} \rightarrow \operatorname{Hom}_{\mathcal{A} \Lambda}(-, M)\right|_{\mathrm{add} \bar{M}^{\prime}} \rightarrow 0
\end{gathered}
$$

é exata.

Denotemos por $K_{i}$ o núcleo de $\varphi_{i}$ para cada $i \in\{1, \ldots, s-1\}$. Temos que $\operatorname{Hom}_{\mathcal{A} \Lambda}(Z \oplus$ $F \oplus N, M)=0$ e $\operatorname{Hom}_{\mathcal{A} \Lambda}\left(Z \oplus F \oplus N, K_{i}\right)=0$ pois $\mathcal{A}$ é fechada para predecessores. Então, a sequência

$$
\begin{gathered}
\left.\left.0 \rightarrow \operatorname{Hom}_{\mathcal{A} \Lambda}\left(-, M_{s}\right)\right|_{\operatorname{add}(Z \oplus F \oplus N)} \rightarrow \operatorname{Hom}_{\mathcal{A} \Lambda}\left(-, M_{s-1}\right)\right|_{\operatorname{add}(Z \oplus F \oplus N)} \rightarrow \cdots \\
\left.\left.\quad \cdots \rightarrow \operatorname{Hom}_{\mathcal{A} \Lambda}\left(-, M_{1}\right)\right|_{\operatorname{add}(Z \oplus F \oplus N)} \rightarrow \operatorname{Hom}_{\mathcal{A} \Lambda}(-, M)\right|_{\operatorname{add}(Z \oplus F \oplus N)} \rightarrow 0
\end{gathered}
$$

é exata.

Finalmente, seja $L$ um módulo indecomponível em add $E$. Como $L$ é Ext-injetivo em $\mathcal{A}$ e não é injetivo em $\bmod \Lambda$ então $\tau_{\Lambda}^{-1} L$ não está em $\mathcal{A}$. Desde que $M \notin \operatorname{add} \bar{X}$, em particular $M$ não é Ext-injetivo e portanto $\tau_{\Lambda}^{-1} M$ está em $\mathcal{A}$. Portanto, $\operatorname{Hom}_{\Lambda}\left(\tau_{\Lambda}^{-1} L, \tau_{\Lambda}^{-1} M\right)=0$ e então $\overline{\operatorname{Hom}}_{\Lambda}(L, M)=0$. Se $f: L \rightarrow M$ é um morfismo então existe um $\Lambda$-módulo injetivo $I$ e morfismos $f_{1}: L \rightarrow I, f_{2}: I \rightarrow M$ tais que $f=f_{2} \circ f_{1}$. Como $I$ é um somando de $\bar{M}$ então $\operatorname{Hom}_{\Lambda}\left(I, M_{1}\right) \rightarrow \operatorname{Hom}_{\Lambda}(I, M) \rightarrow 0$ é exata e, portanto, existe $g: I \rightarrow M_{1}$ tal que $\varphi \circ g=f_{2}$, ou seja, $f=\varphi \circ\left(g \circ f_{1}\right)=\operatorname{Hom}_{\Lambda}(L, \varphi)\left(g \circ f_{1}\right)$ e, segue que, $0 \rightarrow \operatorname{Hom}_{\Lambda}\left(L, K_{1}\right) \rightarrow$ $\operatorname{Hom}_{\Lambda}\left(L, M_{1}\right) \rightarrow \operatorname{Hom}_{\Lambda}(L, M) \rightarrow 0$ é exata.

Pelo Lema 5.1.1 podemos supor que (*) é minimal e pelo Lema 5.4.10 temos que cada $K_{i}$ (que está em add $\mathcal{A}$ ) não contém somandos Ext-injetivos, portanto o mesmo argumento é válido trocando $M$ por $K_{i}$ para cada $i \in\{1, \ldots, s-1\}$. Portanto, para cada $i$ a sequência $0 \rightarrow \operatorname{Hom}_{\Lambda}\left(L, K_{i+1}\right) \rightarrow \operatorname{Hom}_{\Lambda}\left(L, M_{i+1}\right) \rightarrow \operatorname{Hom}_{\Lambda}\left(L, K_{i}\right) \rightarrow 0$ é exata. Isto mostra que

$$
\begin{gathered}
\left.\left.0 \rightarrow \operatorname{Hom}_{\mathcal{A} \Lambda}\left(-, M_{s}\right)\right|_{\operatorname{add} E} \rightarrow \operatorname{Hom}_{\mathcal{A} \Lambda}\left(-, M_{s-1}\right)\right|_{\operatorname{add} E} \rightarrow \cdots \\
\left.\left.\quad \cdots \rightarrow \operatorname{Hom}_{\mathcal{A} \Lambda}\left(-, M_{1}\right)\right|_{\mathrm{add} E} \rightarrow \operatorname{Hom}_{\mathcal{A} \Lambda}(-, M)\right|_{\mathrm{add} E} \rightarrow 0
\end{gathered}
$$

é exata. Logo $(*)$ é uma add $\bar{X}$-resolução de aproximação de comprimento $s$ de $M$. Portanto, rep. $\operatorname{dim}_{\mathcal{A}} \Lambda \leq s+1=\operatorname{rep} \cdot \operatorname{dim} \Lambda$.

Exemplo 5.4.11 Vejamos outra forma de concluir que a dimensão de representação da álgebra $\Lambda$ do Exemplo 5.3.1 é 3 (ver Exemplo 5.3.3).

Como $\Lambda$ é de tipo infinito temos que rep.dim $\Lambda \geq 3$.

A parte direita $\mathcal{R}_{\Lambda}$ consiste de todos os sucessores de $\tau^{-1} P_{4}$ e add $\mathcal{R}_{\Lambda}$ é covariantemente 
finita. A parte esquerda é $\mathcal{L}_{\Lambda}=\left\{P_{1}, P_{2}, S_{2}, P_{3}\right\}$. Sua álgebra suporte ${ }_{\left(\mathcal{L}_{\Lambda}\right)} \Lambda$ é dada pelos objetos 1, 2 e 3, ou seja, $\left(\mathcal{L}_{\Lambda}\right) \Lambda$ é a álgebra inclinada cuja aljava ordinária é $1 \stackrel{\alpha}{\leftarrow} 2 \underset{\beta_{2}}{\leftarrow} 3$ com as relações $\beta_{i} \alpha=0$.

Denote $\mathcal{A}=\operatorname{ind}_{\left(\mathcal{L}_{\Lambda}\right)} \Lambda$ que consiste de todos os predecessores de $S_{3}$ e portanto infinita. Neste caso, é fácil ver que $\left(\mathcal{A},\left(\mathcal{A} \cup \mathcal{R}_{\Lambda}\right)^{c}, \mathcal{R}_{\Lambda}\right)$ é uma trisecção de ind $\Lambda$ com $\left(\mathcal{A} \cup \mathcal{R}_{\Lambda}\right)^{c}$ finita. Pelo Teorema 5.4.7, rep.dim $\Lambda=$ rep.dim ${ }_{\mathcal{A}} \Lambda$. Mas ${ }_{\mathcal{A}} \Lambda={ }_{\left(\mathcal{L}_{\Lambda}\right)} \Lambda$ e portanto rep. $\operatorname{dim} \Lambda=3$.

Como consequências diretas do Teorema 5.4.7 temos os seguintes corolários.

Corolário 5.4.12 Seja $\Lambda$ uma álgebra de tipo infinito.

(a) Se $\mathcal{A}$ é uma subcategoria plena de ind $\Lambda$ cofinita e fechada para predecessores então

$$
\text { rep. } \operatorname{dim} \Lambda=\max \left\{3, \text { rep. } \operatorname{dim}{ }_{\mathcal{A}} \Lambda\right\}
$$

(b) Se $\mathcal{C}$ é uma subcategoria plena de ind $\Lambda$ cofinita e fechada para sucessores então

$$
\text { rep.dim } \Lambda=\max \left\{3, \text { rep.dim } \Lambda_{\mathcal{C}}\right\}
$$

Prova. Para o item (a) basta fazer $\mathcal{B}=\mathcal{A}^{c}$ e $\mathcal{C}=\emptyset$ no Teorema 5.4.7. O item (b) é dual.

Corolário 5.4.13 Seja $\Lambda$ uma álgebra de Artin de tipo infinito.

(a) $S e \mathcal{C} \subseteq \mathcal{R}_{\Lambda}$ é uma subcategoria plena de ind $\Lambda$ fechada para sucessores tal que addC é covariantemente finita, então

$$
\text { rep.dim } \Lambda=\max \left\{3, \text { rep.dim }{ }_{\left(\mathcal{C}^{c}\right)} \Lambda\right\}
$$

(b) $S e \mathcal{A} \subseteq \mathcal{L}_{\Lambda}$ é uma subcategoria plena de ind $\Lambda$ fechada para predecessores tal que add $\mathcal{A}$ é contravariantemente finita, então

$$
\text { rep.dim } \Lambda=\max \left\{3, \text { rep.dim } \Lambda_{\left(\mathcal{A}^{c}\right)}\right\}
$$

Prova. Para (a) basta fazer $\mathcal{A}=\mathcal{C}^{c}$ e $\mathcal{B}=\emptyset$. O item (b) é dual.

Podemos melhorar o resultado deste último corolário, isto é, não precisamos exigir que a subcategoria seja homologicamente finita. Para isso precisaremos do seguinte resultado.

Lema 5.4.14 ([Ass09](8.2)) Sejam $\Lambda$ uma álgebra de Artin, $\mathcal{A}$ uma subcategoria plena de $\mathcal{L}_{\Lambda}$ fechada para predecessores e $E$ a soma direta dos Ext-injetivos de $\mathcal{A}$. Então, add $\mathcal{A}$ é contravariantemente finita se, e somente se, $\mathcal{A}=\operatorname{Pred} E$. 
Proposição 5.4.15 Seja $\Lambda$ uma álgebra de Artin de tipo infinito.

(a) $S e \mathcal{C} \subseteq \mathcal{R}_{\Lambda}$ é uma subcategoria plena de ind $\Lambda$ fechada para sucessores, então

$$
\text { rep.dim } \Lambda=\max \left\{3, \text { rep.dim }{ }_{\left(\mathcal{C}^{c}\right)} \Lambda\right\}
$$

(b) $S e \mathcal{A} \subseteq \mathcal{L}_{\Lambda}$ é uma subcategoria plena de ind $\Lambda$ fechada para predecessores, então

$$
\text { rep.dim } \Lambda=\max \left\{3, \text { rep.dim } \Lambda_{\left(\mathcal{A}^{c}\right)}\right\}
$$

Prova. Se cada $\Lambda$-módulo projetivo indecomponível está em $\mathcal{C}^{c}$ então $\Lambda={ }_{\left(\mathcal{C}^{c}\right)} \Lambda$. Caso contrário, seja $\mathcal{D}=\operatorname{Succ} Y$ onde $Y$ é a soma direta de todos os $\Lambda$-módulos projetivos indecomponíveis que estão em $\mathcal{C}$. Então $\mathcal{D}$ é uma subcategoria plena de $\mathcal{R}_{\Lambda}$ que é fechada para sucessores e se $F$ denota a soma direta de todos os Ext-projetivos indecomponíveis em add $\mathcal{D}$, temos que $\mathcal{D}=\operatorname{Succ} F$, pois, como $Y$ é Ext-projetivo em add $\mathcal{D}$ temos $\mathcal{D}=\operatorname{Succ} Y \subseteq \operatorname{Succ} F$ e, como $F \in \operatorname{add} \mathcal{D}$ e $\mathcal{D}$ é fechada para sucessores, temos $\operatorname{Succ} F \subseteq \mathcal{D}$. Pelo Lema 5.4.14 segue que add $\mathcal{D}$ é covariantemente finita. Pelo Lema 5.4.13 segue que rep.dim $\Lambda=\max \left\{3\right.$, rep.dim $\left.{ }_{\left(\mathcal{D}^{c}\right)} \Lambda\right\}$. Finalmente, para um $\Lambda$-módulo projetivo indecomponível $P$ temos que $P$ está em $\mathcal{C}$ se, e somente se, $P$ está em $\mathcal{D}$ e portanto ${ }_{\left(\mathcal{C}^{c}\right)} \Lambda={ }_{\left(\mathcal{D}^{c}\right)} \Lambda$ o que completa a prova de (a). O item (b) é dual.

Corolário 5.4.16 Se $\Lambda$ é uma álgebra de tipo infinito então

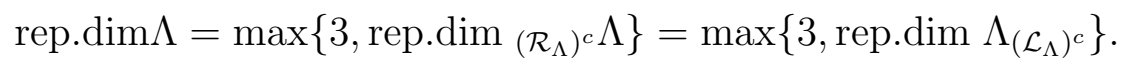

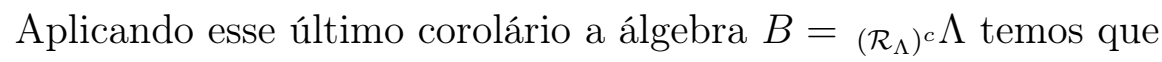

$$
\text { rep.dim } \Lambda=\max \left\{3, \text { rep.dim } B_{\left(\mathcal{L}_{B}\right)^{c}}\right\}
$$

e concluímos que a dimensão de representação da álgebra $\Lambda$ depende de uma subcategoria de ${ }_{\left(\mathcal{R}_{\Lambda}\right)^{c}} \Lambda$ e de $\Lambda_{\left(\mathcal{L}_{\Lambda}\right)^{c}}$.

\subsection{Dimensão de representação das álgebras laura e ada}

Como aplicação dos resultados sobre dimensão de representação apresentados, faremos uma prova curta para o resultado de [APT06](4.1) que diz que se $\Lambda$ é uma álgebra laura então rep.dim $\Lambda \leq 3$. A seguir também provamos que o mesmo resultado é válido para as álgebras ada. 


\section{Dimensão de representação das álgebras laura}

Lembremos que uma álgebra de Artin é dita laura se $\mathcal{L}_{\Lambda} \cup \mathcal{R}_{\Lambda}$ é cofinita em ind $\Lambda$ e que é dita laura estrita se for laura mas não quase inclinada. Se $\Lambda$ é uma álgebra laura estrita, por [ACT04](4.4), temos que $\Lambda$ é suportada à direita e à esquerda, isto é, add $\mathcal{L}_{\Lambda}$ é contravariantemente finita e $\operatorname{add} \mathcal{R}_{\Lambda}$ é covariantemente finita.

Corolário 5.5.1 Se $\Lambda$ é uma álgebra laura então rep.dim $\Lambda \leq 3$.

Prova. Se $\Lambda$ é quase inclinada, o resultado segue de [Opp10], então podemos assumir que $\Lambda$ é laura estrita. Como $\Lambda$ é suportada à esquerda, ou seja, add $\mathcal{L}_{\Lambda}$ é contravariantemente finita e por [ACT04] (5.1) temos que ${ }_{\left(\mathcal{L}_{\Lambda}\right)} \Lambda$ é uma álgebra inclinada então rep.dim ${ }_{\left(\mathcal{L}_{\Lambda}\right)} \Lambda \leq 3$.

Pelo Corolário 5.4.2, como $\left(\mathcal{L}_{\Lambda}, \mathcal{B}, \mathcal{R}_{\Lambda} \backslash \mathcal{L}_{\Lambda}\right)$ é uma trisecção de ind $\Lambda$ onde $\mathcal{B}=\left(\mathcal{L}_{\Lambda} \cup \mathcal{R}_{\Lambda}\right)^{c}$ é finita então $\operatorname{add}\left(\mathcal{R}_{\Lambda} \backslash \mathcal{L}_{\Lambda}\right)$ é covariantemente finita. Suponha que $\Lambda$ é de tipo infinito então, pelo Teorema 5.4.7, temos que rep.dim $\Lambda=\max \left\{3\right.$, rep.dim $\left.\left(\mathcal{L}_{\Lambda}\right) \Lambda\right\}=3$.

\section{Dimensão de representação das álgebras ada}

Teorema 5.5.2 Seja $\Lambda$ uma álgebra de Artin de tipo infinito.

- Se $\Lambda$ está em $\operatorname{add}\left(\mathcal{L}_{\Lambda} \cup \mathcal{R}_{\Lambda}\right)$ então rep.dim $\Lambda=\max \left\{3, \operatorname{rep} \cdot \operatorname{dim}\left(\mathcal{L}_{\Lambda}\right) \Lambda\right\}$.

- Se D $\Lambda$ está em $\operatorname{add}\left(\mathcal{L}_{\Lambda} \cup \mathcal{R}_{\Lambda}\right)$ então rep.dim $\Lambda=\max \left\{3, \operatorname{rep} \cdot \operatorname{dim} \Lambda_{\left(\mathcal{R}_{\Lambda}\right)}\right\}$.

Prova. Suporemos que $\Lambda$ está em $\operatorname{add}\left(\mathcal{L}_{\Lambda} \cup \mathcal{R}_{\Lambda}\right)$. O outro caso se prova de forma análoga. Consideremos $\mathcal{C}=\mathcal{R}_{\Lambda} \backslash \mathcal{L}_{\Lambda}$.

Pela Proposição 5.4.15, temos que rep.dim $\Lambda=\max \left\{3\right.$, rep.dim $\left.{ }_{\left(\mathcal{C}^{c}\right)} \Lambda\right\}$. Pela hipótese, temos que cada projetivo indecomponível está em $\mathcal{L}_{\Lambda} \cup \mathcal{R}_{\Lambda}, \operatorname{logo}{ }_{\left(\mathcal{C}^{c}\right)} \Lambda={ }_{\left(\mathcal{L}_{\Lambda}\right)} \Lambda$.

Corolário 5.5.3 Se $\Lambda$ é uma álgebra ada então rep.dim $\Lambda \leq 3$.

Prova.

Do teorema anterior segue que rep. $\operatorname{dim} \Lambda=\max \left\{3, \operatorname{rep} \cdot \operatorname{dim}\left(\mathcal{L}_{\Lambda}\right) \Lambda\right\}$. Por [ACLV12] $(4.1)$ a álgebra $\left(\mathcal{L}_{\Lambda}\right) \Lambda$ é produto de álgebras inclinadas, então rep.dim( ${ }_{\left(\mathcal{L}_{\Lambda}\right)} \Lambda \leq 3$ e portanto rep.dim $\Lambda=3$.

\subsection{Aplicações ao produto fibrado orientado}

Lembremos das subcategorias $\mathcal{A}, \mathcal{B}$ e $\mathcal{C}$ definidas em 4.2 para um produto fibrado orientado.

$$
\mathcal{A}=\operatorname{Pred} D A^{\prime}, \mathcal{C}=\operatorname{Succ} C^{\prime} \text { e } \mathcal{B}=\operatorname{ind} R \backslash(\mathcal{A} \cup \mathcal{C})
$$

Como aplicação do Teorema 5.3.2 e da Proposição 5.4.15 temos a seguinte proposição. 
Proposição 5.6.1 Seja $R$ o produto fibrado orientado de $A \rightarrow B$ e $C \rightarrow B$ com $B$ uma álgebra hereditária. Se ind $A$ é fechada para predecessores ou ind $C$ é fechada para sucessores, então

$$
\text { rep. } \operatorname{dim} R \leq \max \{\text { rep. } \operatorname{dim} A, \operatorname{rep} \cdot \operatorname{dim} C\}
$$

Prova. Suponhamos que ind $C$ é fechada para sucessores. Do Corolário 4.2.3, temos que $\operatorname{ind} R=\mathcal{A} \cup \operatorname{ind} C$ e, portanto, $\mathcal{A} \backslash$ ind $C$ é fechada para predecessores. Podemos considerar a seguinte trisecção $(\mathcal{A} \backslash$ ind $C, \emptyset$, ind $C$ ) para ind $R$. Pelo Teorema 5.3 .2 (a), rep.dim $R \leq$ $\max \left\{\operatorname{rep} \cdot \operatorname{dim}_{(\mathcal{A} \backslash \text { ind } C)} R\right.$, rep.dim $\left.C\right\}$, pois $R_{\text {ind } C}=C$. Além disso, da Proposição 3.2 .9 segue que $P(x) \in \mathcal{A} \backslash \operatorname{ind} C$ se, e somente se, $P(x) \in(\operatorname{ind} A \backslash \operatorname{ind} B) \cap \mathcal{A}$ e, portanto, temos que $(\mathcal{A} \backslash \operatorname{ind} C) R=(\mathcal{A} \backslash \operatorname{ind} C) A$.

Como ind $C$ é fechada para sucessores em ind $R$, segue que ind $B$ é fechada para sucessores em ind $A$. Como $I_{A}(i)=I_{B}(i)$ para cada $i \in\left(Q_{B}\right)_{0}$ segue que, $\operatorname{di}_{A} M=\operatorname{di}_{B} M$ para $M \in \operatorname{ind} B$. Como $B$ é hereditária, segue que $\operatorname{ind} B \subseteq \mathcal{R}_{A}$. Pela Proposição 5.4.15, temos que rep.dim $A=\max \left\{3, \operatorname{rep} \cdot \operatorname{dim}_{(\operatorname{ind} B)^{c}} A\right\}$. Mas, $(\operatorname{ind} B)^{c} A={ }_{(\mathcal{A} \backslash \operatorname{ind} C)} A$ e, portanto, rep. $\operatorname{dim}_{(\mathcal{A} \backslash \text { ind } C)} R=$ rep. $\operatorname{dim}_{(\mathcal{A} \backslash \text { ind } C)} A \leq \operatorname{rep} \cdot \operatorname{dim} A$.

Portanto, rep.dim $R \leq \max \{$ rep. $\operatorname{dim} A, \operatorname{rep} \cdot \operatorname{dim} C\}$.

Se supormos ind $A$ fechada para predecessores, o resultado segue, por dualidades.

Corolário 5.6.2 Seja $R$ o produto fibrado Nakayama orientado de $A \rightarrow B$ e $C \rightarrow B$. Então

$$
\text { rep. } \operatorname{dim} R \leq \max \{\text { rep. } \operatorname{dim} A, \operatorname{rep} \cdot \operatorname{dim} C\}
$$

Prova. Do Corolário 4.3.4, temos que ind $C$ é fechada para sucessores. Além disso, pela Definição 3.3.2 temos que $B$ é hereditária. O resultado segue da proposição anterior.

Exemplo 5.6.3 Seja $R$ a álgebra dada pela aljava

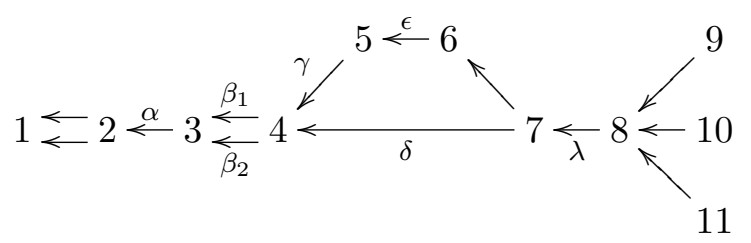

com as relações $\beta_{i} \alpha=\gamma \beta_{i}=\delta \beta_{i}=\epsilon \gamma=\lambda \delta=0$. Podemos ver $R$ como um produto fibrado Nakayama orientado onde $B$ é dado por $2 \stackrel{\alpha}{\longleftarrow} 3$, A é dado por $1 \longleftarrow 2 \stackrel{\alpha}{\longleftarrow} 3$ e $C$ é dado por

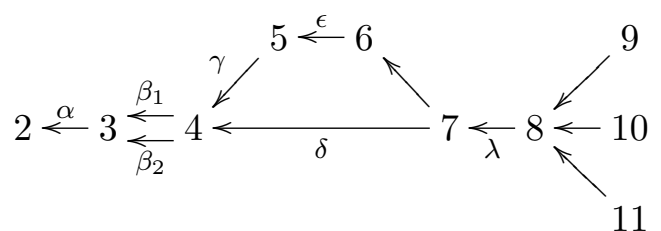


com as mesmas relações de $R$.

Como A é hereditária de tipo infinito então rep.dim $A=3$. A álgebra $C$ é a mesma daquela do Exemplo 5.3.1 e então rep.dimC $=3$. Pelo último corolário segue que $\operatorname{rep} \cdot \operatorname{dim} R=3$.

Lema 5.6.4 Seja $R$ o produto fibrado orientado de $A \rightarrow B$ e $C \rightarrow B$. Então, $A={ }_{(\mathcal{C})^{c}} R$ e $C=R_{(\mathcal{A})^{c}}$.

Prova. Da definição de $\mathcal{A}, \mathcal{B}$ e $\mathcal{C}$ temos que $(\mathcal{C})^{c}=\mathcal{B} \cup(\mathcal{A} \backslash \mathcal{C})$ e, da Proposição 4.2.2, segue que $(\mathcal{C})^{c} \subseteq \operatorname{ind} A$. Portanto, se $P(x) \in(\mathcal{C})^{c}$ então $P(x) \in \operatorname{ind} A$ e portanto $x \in\left(Q_{A}\right)_{0}$. Por outro lado, se $x \in\left(Q_{A}\right)_{0}$, pelo Lema 3.2.9, $P(x)=P_{A}(x) \in \operatorname{ind} A$. Se $P(x) \in \mathcal{C}$ então existem $y \in\left(Q_{C}\right)_{0} \backslash\left(Q_{B}\right)_{0}$ e um caminho de $P(y)$ para $P(x)$. Isso implica na existência de um caminho de $x$ para $y$ na aljava $Q_{R}$, o que é uma contradição com a orientação de $Q_{R}$ dada pela Definição 3.2.1. Logo, $P(x) \in(\mathcal{C})^{c}$ e portanto $\left.A={ }_{(\mathcal{C}}\right)^{c} R$.

A outra igualdade se mostra de forma análoga.

Proposição 5.6.5 Seja $R$ o produto fibrado orientado de $A \rightarrow B$ e $\rightarrow B$ com $\mathcal{A}$ e $\mathcal{C}$ disjuntas.

(a) Se $C$ é hereditária então $\operatorname{rep} \cdot \operatorname{dim} R \leq \max \{3, \operatorname{rep} \cdot \operatorname{dim} A\}$.

(b) Se A é hereditária então $\operatorname{rep} \cdot \operatorname{dim} R \leq \max \{3, \operatorname{rep} \cdot \operatorname{dim} C\}$.

Prova. Se $R$ é de tipo finito não há o que fazer. Suponha que $R$ é de tipo infinito. Se $C$ é hereditária então $\operatorname{di} M=\operatorname{di}_{C} M \leq 1$ para todo $M$ em $\mathcal{C} \backslash \mathcal{A}$, que é fechada para sucessores. $\operatorname{Logo},(\mathcal{C} \backslash \mathcal{A}) \subseteq \mathcal{R}_{\mathcal{R}}$. Pela Proposição 5.4 .15 segue que rep.dim $R \leq \max \left\{3, \operatorname{rep} \cdot \operatorname{dim}{ }_{(\mathcal{C} \backslash \mathcal{A})^{c}} R\right\}$. Como $\mathcal{A}$ e $\mathcal{C}$ são disjuntas então $\mathcal{C} \backslash \mathcal{A}=\mathcal{C}$ e, pelo lema anterior, ${ }_{(\mathcal{C} \backslash \mathcal{A})^{c}} R=A$ concluíndo o item (a). A prova de (b) é análoga. 
DIMENSÃO DE REPRESENTAÇÃO 


\section{Apêndice A}

\section{Exemplo}

Segue um esquema da aljava de Auslander-Reiten da álgebra que aparece nos exemplos 5.3.1, 5.3.3 e 5.4.11, para facilitar a visualização desses exemplos. A álgebra é dada pela aljava

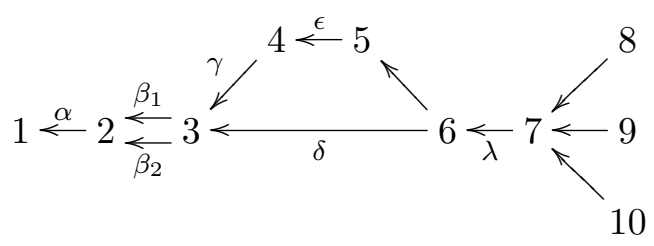

com as relações $\beta_{i} \alpha=\gamma \beta_{i}=\delta \beta_{i}=\epsilon \gamma=\lambda \delta=0$. 
90 APÊNDICE A
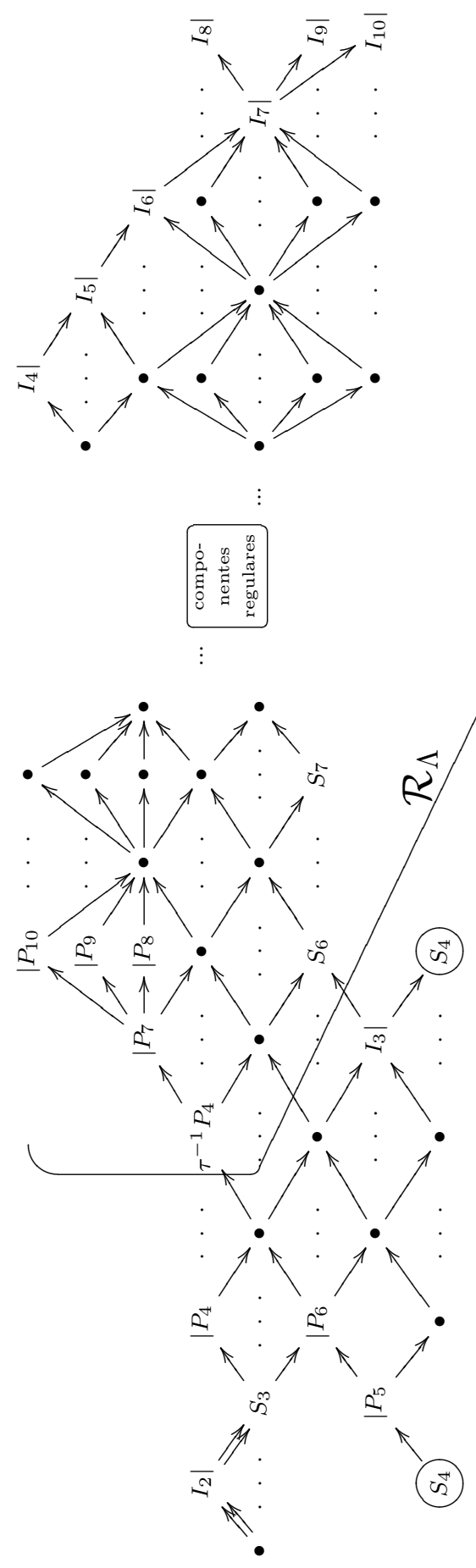

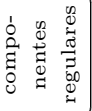

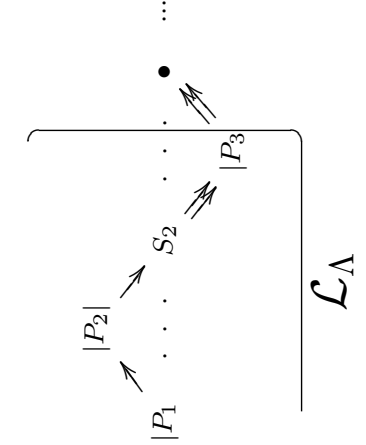




\section{Referências Bibliográficas}

$\left[\mathrm{AAC}^{+} 11\right]$ E. R. Alvares, I. Assem, F. U. Coelho, M. I. Peña e S. Trepode. From trisections in module categories to quasi-directed componentes. J. Algebra and its Appl., 10(3):409-433, 2011. 2, 53

[AC94] I. Assem e F. U. Coelho. Glueings of tilted algebras. Journal of Pure and Applied Algebra, 96(3):225-243, 1994. 16

[AC03] I. Assem e F. U. Coelho. Two-sided gluings of tilted algebras. J. Algebra, 269:456479, 2003. 1,16

[AC04] I. Assem e F. U. Coelho. Endomorphism rings of projective modules over laura algebras. J. Algebra and its Appl., 3(1):49-60, 2004. 63

$\left[\mathrm{ACL}^{+} 05\right]$ I. Assem, F. U. Coelho, M. Lanzilotta, D. Smith e S. Trepode. Algebras determined by their left and right parts. Em Algebraic Structures and their representations, volume 376, páginas 13-47. American Math. Soc., 2005. 14

[ACLV12] I. Assem, D. Castonguay, M. Lanzilotta e R. S. Vargas. Algebras determined by their supports. J. Pure Appl. Algebra, 216:1134-1145, 2012. 2, 16, 56, 63, 85

[ACPV08] I. Assem, J. Cappa, M. I. Platzeck e M. Verdecchia. Modulos inclinantes y algebras inclinadas, volume 21 of Notas de Algebra y Análises. Universidad Nacional del Sur, Bahia Blanca - Argentina, 2008. 15

[ACT04] I. Assem, F. U. Coelho e S. Trepode. The left and right parts of a module category. J. Algebra, 281:426-439, 2004. 85

[ACT09] I. Assem, F. U. Coelho e S. Trepode. Contravariantly finite subcategories closed under predecessors. J. Algebra, 322:1196-1213, 2009. 54, 76

[APT06] I. Assem, M. I. Platzeck e S. Trepode. On the representation dimension of tilted and laura algebras. J. Algebra, páginas 426-439, 2006. 3, 70, 76, 84

[ARS95] M. Auslander, I. Reiten e S. Smalø. Representation theory of artin algebra, volume 36 of Cambridge Studies in Advanced Mathematics. Cambridge Univ. Press, 1995. $7,68,80$

[AS80] M. Auslander e S. Smalø. Preprojective modules over artin algebras. J. Algebra, 66(1):61-122, 1980. 3, 75

[AS81] M. Auslander e S. Smalø. Almost split sequences in subcategories. J. Algebra, $69: 426-454,1981.75$

[Ass90] I. Assem. Tilting theory - an introduction. Em Topics in algebra, volume 26 of Banach Center Publ., páginas 127-180. PWN, Warsaw, Poland, 1990. 15 
[Ass97] I. Assem. Algèbres et modules. Masson - Les Presses de l'Université d'Ottawa, 1997. 7, 33, 37, 69

[ASS06] I. Assem, D. Simson e A. Skowroński. Elements of the representation theory of associative algebras. Vol. 1, volume 65 of London Mathematical Society Student Texts. Cambridge University Press, Cambridge, 2006. 7, 11, 14, 19, 66

[Ass09] I. Assem. Left sections and the left part of an artin algebra. Colloq. Math., 116(2):273-300, 2009. 2, 83

[Aus71] M. Auslander. Representation dimension of artin algebras. Queen Mary College Mathematical Notes, London, 1971. 3, 67, 70

[CL99] F. U. Coelho e M. Lanzilotta. Algebras with small homological dimensions. Manuscripta Math., 100:1-11, 1999. 1, 15, 56

[CL03] F. U. Coelho e M. Lanzilotta. Weakly shod algebras. J. Algebra, 265(1):379-403, 2003. 1

[Coe92] F. U. Coelho. Uma introdução à teoria de representações de álgebras (minicurso). Em Atas da XII Escola de Álgebra, página 60p, Diamantina, 1992. 7, 11

[CP04] F. U. Coelho e M. I. Platzeck. On the representation dimension of some classes of algebras. J. Algebra, 275:615-628, 2004. 3, 70

[EHIS04] K. Erdmann, T. Holm, O. Iyama e J. Schröer. Radical embeddings and representation dimension. Adv. Math., 185(1):159-177, 2004. 70

[Ehr79] H. Ehrig. Introduction to the algebraic theory of graph grammars. Lecture Notes in Comput. Sci., 73:1-69, 1979. 39

[FV85] A. Facchini e P. Vámos. Injective modules over pullbacks. J. London Math. Soc., páginas 425-438, 1985. 1

[HR82] D. Happel e C. M. Ringel. Tilted algebras. Trans. Amer. Math. Soc., 274(2):399443, 1982. 1,15

[HRS96] D. Happel, I. Reiten e S. Smalø. Tilting in abelian categories and quasitilted algebras. Mem. of AMS, 120, 1996. 1, 2, 15

[IPTZ87] K. Igusa, M. I. Platzeck, G. Todorov e D. Zacharia. Auslander algebras of finite representation type. Comm. Algebra, 15(1-2):377-424, 1987. 1, 2, 17, 31, 40, 47, 51

[IT05] K. Igusa e G. Todorov. On the finitistic global dimension conjecture for Artin algebras. Em Representations of algebras and related topics, volume 45 of Fields Inst. Commun., páginas 201-204. Amer. Math. Soc., Providence, RI, 2005. 3, 67

[Iya03] O. Iyama. Finiteness of representation dimension. Proc. Amer. Math. Soc., 131(4):1011-1014, 2003. 3, 67

[Lev04] J. Levesque. Produits fibrés d'algèbres et inclinaison. Tese de Doutorado, Université de Sherbrooke, Sherbrooke, 2004. 2, 31, 39, 40, 43, 47, 48, 59 
[Lev08] J. Levesque. Nakayama oriented pullbacks and stably hereditary algebras. J. Pure Appl. Algebra, 212:1149-1161, 2008. 1, 2, 5, 31, 48, 53, 65

[Mil71] J. W. Milnor. Introduction to algebraic k-theory. Em Annals of Mathematics Studies, volume 72. Princeton Univ. Press., 1971. 1

[Opp10] S. Oppermann. Representation dimension of quasi-tilted algebras. J. Lond. Math. Soc. (2), 81(2):435-456, 2010. 3, 85

[Pog01] Z. Pogorzaly. Modules over pullbacks of algebras. Comm. Algebra, 29(4):17091728, 2001. 35, 36, 37

[Rin08] C. M. Ringel. An introduction to the representation dimension of artin algebras. (www.math.uni-bielefeld.de/ ringel/lectures.html), February 2008. 3

[Rot79] J. Rotman. An introduction to homological algebra. Academic Press, Inc., 1979. 32

[Rou06] R. Rouquier. Representation dimension of exterior algebras. Invent. Math., 165(2):357-367, 2006. 3, 67

[Sma84] S. Smalø. Torsion theories and tilting modules. Bull. London Math. Soc., 16(5):518-522, 1984. 75

[Wis85] A. N. Wiseman. Projective modules over pullback rings. Math. Proc. Cambridge Philos. Soc., 97(3):399-406, 1985. 1, 46

[Xi02] C. Xi. Representation dimension and quasi-hereditary algebras. Adv. Math., 168:193-212, 2002. 70 